\title{
On the origin of approximate custodial symmetry in the Two-Higgs Doublet Model.
}

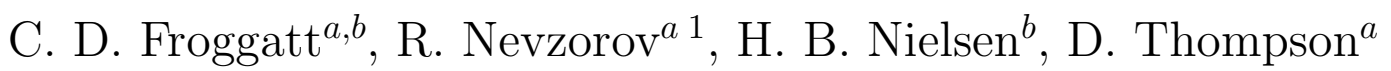 \\ ${ }^{a}$ Department of Physics and Astronomy, \\ Glasgow University, Glasgow, Scotland \\ ${ }^{b}$ The Niels Bohr Institute, Copenhagen, Denmark
}

\begin{abstract}
We argue that the consistent implementation of the multiple point principle (MPP) in the general non-supersymmetric two Higgs doublet model (2HDM) can lead to a set of approximate global custodial symmetries that ensure CP conservation in the Higgs sector and the absence of flavour changing neutral currents (FCNC) in the considered model. In particular the existence of a large set of degenerate vacua at some high energy scale $\Lambda$ caused by the MPP can result in approximate $U(1)$ and $Z_{2}$ symmetries that suppress $\mathrm{FCNC}$ and $\mathrm{CP}$-violating interactions in the $2 \mathrm{HDM}$. We explore the renormalisation group (RG) flow of the Yukawa and Higgs couplings within the MPP inspired 2HDM with approximate custodial symmetries and show that the solutions of the RG equations are focused near quasi-fixed points at low energies if the MPP scale scale $\Lambda$ is relatively high. We study the Higgs spectrum and couplings near the quasi-fixed point at moderate values of $\tan \beta$ and compute a theoretical upper bound on the lightest Higgs boson mass. If $\Lambda \gtrsim 10^{10} \mathrm{GeV}$ the lightest $\mathrm{CP}$-even Higgs boson is always lighter than $125 \mathrm{GeV}$. When the MPP scale is low, the mass of the lightest Higgs particle can reach $180-220 \mathrm{GeV}$ while its coupling to the top quark can be significantly larger than in the SM, resulting in the enhanced production of Higgs bosons at the LHC. Other possible scenarios that appear as a result of the implementation of the MPP in the 2HDM are also discussed.
\end{abstract}

\footnotetext{
${ }^{1}$ On leave of absence from the Theory Department, ITEP, Moscow, Russia
} 


\section{Introduction}

The understanding of the origin of the strong suppression of flavour changing neutral current (FCNC) transitions observed in Nature together with the origin of CP violation, are among the major outstanding problems in particle physics. In the standard model (SM) CP violation arises from the phase of the CKM matrix [1]-[2] and from the " $\theta$ term" in the QCD Lagrangian. Within the SM the particle content, gauge invariance and renormalizability imply the absence of FCNC transitions at the tree level. At oneloop, they are further suppressed by light quark masses (when compared to $M_{W}$ ), i.e. through the GIM mechanism [3], and by small mixing between the third and the first two generations.

However because of the possible presence of new physics the SM should be regarded as an effective "low energy" theory which, up to some scale $\Lambda$, is a good approximation to the more fundamental underlying theory. Therefore the renormalizable interactions of the SM are in general supplemented by higher dimensional interaction terms suppressed by some powers of the scale $\Lambda$. These new interactions introduce new sources of $\mathrm{CP}$ violation. In the considered case $S U(3)_{C} \times S U(2)_{W} \times U(1)_{Y}$ invariance is not sufficient any more to protect the observed strong suppression of the FCNC processes. Under these circumstances we may expect that either the scale $\Lambda$ is huge 1 or dangerous new interactions are absent because of symmetries of the underlying theory. If the only suppression of FCNC processes is due to the scale $\Lambda$, then there is a tension between the new physics scale which is required in order to solve the hierarchy problem and the one which is needed in order to satisfy the experimental bounds from flavour physics. This is the so-called new physics flavour problem [4].

In this article we consider the multiple point principle (MPP) [5]-[7] as a possible mechanism for the suppression of the flavour changing neutral current and $\mathrm{CP}$-violation effects within the general non-supersymmetric two Higgs doublet extension of the SM [8]-[9]. The violation of $\mathrm{CP}$ invariance and the existence of tree-level flavour-changing neutral currents are generic features of $S U(2)_{W} \times U(1)_{Y}$ theories with two and more Higgs doublets. Potentially large FCNC interactions appear in these models, because the diagonalization of the quark mass matrix does not automatically lead to the diagonalization of the two or even more Yukawa coupling matrices, which describe the interactions of Higgs bosons with fermionic matter. Moreover the Higgs potential of the two-Higgs doublet model $(2 \mathrm{HDM})$ contains a lot of new couplings. Some of them may be complex, resulting

\footnotetext{
${ }^{1}$ The strongest bounds are obtained from $K^{0}-\overline{K^{0}}$ mixing and $\mathrm{CP}$ violation in $K$ meson decay measurements that forbid any $\Lambda$ below $10^{4} \mathrm{TeV}$. The measurements of $\mathrm{CP}$ violation in $B$ meson decay as well as in $D^{0}-\overline{D^{0}}$ and $B^{0}-\overline{B^{0}}$ mixings imply that $\Lambda \gtrsim 10^{3} \mathrm{TeV}[4$.
} 
in $\mathrm{CP}$ violation in the Higgs sector [10]-[15]. Although one can eliminate the violation of CP invariance in the Higgs sector and tree-level FCNC transitions by imposing a discrete $Z_{2}$ symmetry, such a symmetry leads to the formation of domain walls in the early Universe [16] which create unacceptably large anisotropies in the cosmic microwave background radiation [17]. Therefore in practice it is necessary to impose only an approximate symmetry, typically broken by soft mass terms.

The MPP postulates the existence of many phases with the same energy density which are allowed by a given theory [5]-[7]. When applied to the SM, the multiple point principle implies that the Higgs effective potential possesses two degenerate minima taken to be at the electroweak and Planck scales respectively. The degeneracy of vacua at the electroweak and Planck scales can be achieved only if (see [18])

$$
M_{t}=173 \pm 4 \mathrm{GeV}, \quad M_{H}=135 \pm 9 \mathrm{GeV}
$$

This MPP prediction for the Higgs mass lies on the SM vacuum stability curve [9], [19][31] corresponding to the cut-off $\Lambda=M_{P l}$ 2. The hierarchy between the electroweak and Planck scales might also be explained by MPP within the pure SM, if there exists a third degenerate vacuum [32]-34].

If we require the vacuum we live in to be just metastable w.r.t. decay into the second vacuum, rather than being exactly degenerate with it, and otherwise make similar assumptions to those in [18], the energy density in the second vacuum falls below that of the vacuum in which we live. Consequently the Higgs mass is then predicted to be a bit smaller. With the value used in this article for the top quark mass [35], $M_{t}=171.4 \pm 2.1$ $\mathrm{GeV}$, the value predicted for the Higgs mass from borderline metastability of our vacuum, which we call meta-MPP [36], becomes $M_{H}=118.4 \pm 5 \mathrm{GeV}$. This is remarkably close to the two-standard deviation hint of a Higgs signal seen in LEP [37] at $115 \mathrm{GeV}$.

In previous papers [38]-[40] the MPP assumption has been adapted to models based on $(N=1)$ local supersymmetry - supergravity, in order to provide an explanation for the small deviation of the cosmological constant from zero. Recently we also considered the application of the MPP to the SUSY inspired two Higgs doublet model of type II [41]. We established MPP conditions in this model and discussed the restrictions on the mass of the SM-like Higgs boson caused by the MPP. Here we are going to extend this analysis to the general 2HDM.

In the next section we specify the model. In section 3 we present the derivation of the MPP conditions that result in approximate custodial $U(1)$ and $Z_{2}$ symmetries. The renormalisation group (RG) flow of Yukawa couplings within these MPP inspired two

\footnotetext{
${ }^{2}$ The requirement of the validity of perturbation theory up to the Planck scale leads to an upper bound on $M_{H}$ in the SM, which is about $180-190 \mathrm{GeV}[19]-[23$.
} 
Higgs doublet models is considered in section 4. In particular, we establish the positions of quasi-fixed points and argue that the quasi-fixed point scenarios with large $\tan \beta$ lead to unacceptably large values of the top quark mass. In section 5 we study the evolution of Higgs self-couplings and analyse the spectrum of Higgs bosons and their couplings near the quasi-fixed point at moderate $\tan \beta$. We examine the phenomenological viability of other possible MPP solutions in section 6. Our results are summarised in section 7 . In Appendix A the $\beta$-functions of Higgs self-couplings in the general two Higgs doublet extension of the SM are presented. The derivation of the other MPP conditions that do not give rise to an approximate custodial $U(1)$ symmetry is discussed in Appendix B.

\section{Two Higgs doublet extension of the SM}

The most general renormalizable $S U(2)_{W} \times U(1)_{Y}$ gauge invariant potential of the model involving two Higgs doublets is given by

$$
\begin{gathered}
V_{\text {eff }}\left(H_{1}, H_{2}\right)=m_{1}^{2}(\Phi) H_{1}^{\dagger} H_{1}+m_{2}^{2}(\Phi) H_{2}^{\dagger} H_{2}-\left[m_{3}^{2}(\Phi) H_{1}^{\dagger} H_{2}+\text { h.c. }\right]+ \\
\frac{\lambda_{1}(\Phi)}{2}\left(H_{1}^{\dagger} H_{1}\right)^{2}+\frac{\lambda_{2}(\Phi)}{2}\left(H_{2}^{\dagger} H_{2}\right)^{2}+\lambda_{3}(\Phi)\left(H_{1}^{\dagger} H_{1}\right)\left(H_{2}^{\dagger} H_{2}\right)+\lambda_{4}(\Phi)\left|H_{1}^{\dagger} H_{2}\right|^{2} \\
+\left[\frac{\lambda_{5}(\Phi)}{2}\left(H_{1}^{\dagger} H_{2}\right)^{2}+\lambda_{6}(\Phi)\left(H_{1}^{\dagger} H_{1}\right)\left(H_{1}^{\dagger} H_{2}\right)+\lambda_{7}(\Phi)\left(H_{2}^{\dagger} H_{2}\right)\left(H_{1}^{\dagger} H_{2}\right)+\text { h.c. }\right]
\end{gathered}
$$

where

$$
H_{n}=\left(\begin{array}{c}
\chi_{n}^{+} \\
\left(H_{n}^{0}+i A_{n}^{0}\right) / \sqrt{2}
\end{array}\right) \quad n=1,2 .
$$

It is easy to see that the number of couplings in the two Higgs doublet model potential compared with the SM grows from two to ten. Furthermore, four of them $m_{3}^{2}, \lambda_{5}, \lambda_{6}$ and $\lambda_{7}$ can be complex, inducing $\mathrm{CP}$-violation in the Higgs sector. In what follows we suppose that the mass parameters $m_{i}^{2}$ and Higgs self-couplings $\lambda_{i}$ of the effective potential (2) only depend on the overall sum of the squared norms of the Higgs doublets, i.e.

$$
\Phi^{2}=\Phi_{1}^{2}+\Phi_{2}^{2}, \quad \Phi_{n}^{2}=H_{n}^{\dagger} H_{n}=\frac{1}{2}\left[\left(H_{n}^{0}\right)^{2}+\left(A_{n}^{0}\right)^{2}\right]+\left|\chi_{n}^{+}\right|^{2} .
$$

The dependence of $m_{i}^{2}$ and $\lambda_{i}$ on $\Phi$ is described by the renormalization group equations, where the renormalization scale is replaced by $\Phi$.

At the physical minimum of the scalar potential (2) the Higgs fields develop vacuum expectation values

$$
<H_{1}^{0}>=v_{1}, \quad<H_{2}^{0}>=v_{2}
$$

breaking the $S U(2)_{W} \times U(1)_{Y}$ gauge symmetry to $U(1)_{e m}$ associated with electromagnetism and generating the masses of all bosons and fermions. The overall Higgs norm 
$<\Phi>=\sqrt{\frac{\left|v_{1}\right|^{2}+\left|v_{2}\right|^{2}}{2}}=\frac{v}{\sqrt{2}}=174 \mathrm{GeV}$ is fixed by the Fermi scale. At the same time the ratio of the Higgs vacuum expectation values remains arbitrary. Hence it is convenient to introduce $\tan \beta=\left|v_{2}\right| /\left|v_{1}\right|$.

As has been already mentioned in the Introduction, the Yukawa interactions of the Higgs fields $H_{1}$ and $H_{2}$ with quarks and leptons generate phenomenologically unwanted FCNC transitions. In particular these interactions contribute to the amplitude of $K^{0}-\bar{K}^{0}$ oscillations and give rise to new channels of muon decay like $\mu \rightarrow e^{-} e^{+} e^{-}$. The common way to suppress flavour changing processes is to impose a certain protecting custodial $Z_{2}$ symmetry that forbids potentially dangerous couplings of the Higgs fields to quarks and leptons [42]. Such a custodial symmetry requires the vanishing of the Higgs couplings $\lambda_{6}$ and $\lambda_{7}$. It also requires the down-type quarks to couple to just one Higgs doublet, $H_{1}$ say, while the up-type quarks couple either to the same Higgs doublet $H_{1}$ (Model I) or to the second Higgs doublet $H_{2}$ (Model II) but not both 3 . In fact, as we shall use in subsection 3.3 , it is possible to generalise the idea of such a $Z_{2}$ symmetry so that each fermion couples to just one Higgs field $\left(H_{1}\right.$ or $\left.H_{2}\right)$ but in a generation dependent way. The custodial $Z_{2}$ symmetry forbids the mixing term $m_{3}^{2}(\Phi)\left(H_{1}^{\dagger} H_{2}\right)$ in the Higgs effective potential (2). But usually a soft violation of the $Z_{2}$ symmetry by dimension-two terms is allowed, since it does not induce Higgs-mediated tree-level flavor changing neutral currents (FCNC).

The set of RG equations that determines the running of Yukawa and Higgs couplings in the two Higgs doublet model with exact and softly broken $Z_{2}$ symmetry can be found in [43]-[47]. The constraints on the Higgs masses in the 2HDM with an unbroken $Z_{2}$ symmetry have been examined in a number of publications [46]-[54]. The analysis of [54] was performed assuming vacuum stability and the applicability of perturbation theory up to a high energy scale (of order the grand unification scale), revealing that then all Higgs boson masses lie below $200 \mathrm{GeV}$. A very stringent restriction on the masses of the charged and pseudoscalar states was found. They do not exceed $150 \mathrm{GeV}$. However such a light charged Higgs boson is ruled out by the direct searches for the rare B-meson decays $\left(B \rightarrow X_{s} \gamma\right)$ in the Model II of the 2HDM, which cannot therefore be valid with an unbroken $Z_{2}$ symmetry up to the unification scale. The theoretical restrictions on the mass of the SM-like Higgs boson within the $2 \mathrm{HDM}$ with a softly broken $Z_{2}$ symmetry were studied in [55].

We emphasize that, in this article, we do not impose any custodial symmetry but rather consider the general Higgs potential (2). Instead we require that at some high energy scale $\left(M_{Z}<<\Lambda \lesssim M_{P l}\right)$, which we shall refer to as the MPP scale $\Lambda$, a large set

\footnotetext{
${ }^{3}$ Similarly the leptons are required to only couple to one Higgs doublet, usually chosen to be the same as the down-type quarks. However there are variations of Models I and II, in which the leptons couple to $H_{2}$ rather than to $H_{1}$.
} 
of degenerate vacua allowed by the $2 \mathrm{HDM}$ is realized. In compliance with the MPP, these vacua and the physical one must have the same energy density. Thus the MPP implies that the couplings $\lambda_{i}(\Lambda)$ should be adjusted so that an appropriate cancellation among the quartic terms in the effective potential (2) takes place.

Here and further we impose a certain hierarchical structure on the Yukawa couplings. To explain the observed mass hierarchy in the quark and lepton sectors, we assume that the Yukawa couplings of the quarks and leptons of the third generation are considerably larger than the quark and lepton Yukawa couplings of the first two generation. In this approximation the part of the 2HDM Lagrangian describing the interactions of quarks and leptons with the Higgs doublets $H_{1}$ and $H_{2}$ reduces to

$$
\begin{aligned}
\mathcal{L}_{Y u k} \simeq h_{t}\left(H_{2} \varepsilon Q\right) \bar{t}_{R}+g_{b}\left(H_{2}^{\dagger} Q\right) \bar{b}_{R}+g_{\tau}\left(H_{2}^{\dagger} L\right) \bar{\tau}_{R}+ \\
+g_{t}\left(H_{1} \varepsilon Q\right) \bar{t}_{R}+h_{b}\left(H_{1}^{\dagger} Q\right) \bar{b}_{R}+h_{\tau}\left(H_{1}^{\dagger} L\right) \bar{\tau}_{R}+\text { h.c. },
\end{aligned}
$$

where $Q$ and $L$ are left-handed doublets of quarks and leptons of the third generation, while $\tau_{R}, t_{R}$ and $b_{R}$ are right-handed $S U(2)_{W}$ singlet components of $\tau$-lepton, $t$ - and $b$ quarks. The running of the Yukawa couplings of the third generation obey the following set of differential equations:

$$
\begin{aligned}
\frac{d g_{t}}{d t} & =\frac{1}{16 \pi^{2}}\left[g_{t}\left(\frac{9}{2}\left|g_{t}\right|^{2}+\frac{9}{2}\left|h_{t}\right|^{2}+\frac{3}{2}\left|h_{b}\right|^{2}+\frac{1}{2}\left|g_{b}\right|^{2}+\left|h_{\tau}\right|^{2}\right)+h_{t}\left(g_{b} h_{b}^{*}+g_{\tau} h_{\tau}^{*}\right)-\right. \\
& \left.-g_{t}\left(8 g_{3}^{2}+\frac{9}{4} g_{2}^{2}+\frac{17}{12} g_{1}^{2}\right)\right], \\
\frac{d h_{t}}{d t} & =\frac{1}{16 \pi^{2}}\left[h_{t}\left(\frac{9}{2}\left|g_{t}\right|^{2}+\frac{9}{2}\left|h_{t}\right|^{2}+\frac{1}{2}\left|h_{b}\right|^{2}+\frac{3}{2}\left|g_{b}\right|^{2}+\left|g_{\tau}\right|^{2}\right)+g_{t}\left(h_{b} g_{b}^{*}+h_{\tau} g_{\tau}^{*}\right)-\right. \\
& \left.-h_{t}\left(8 g_{3}^{2}+\frac{9}{4} g_{2}^{2}+\frac{17}{12} g_{1}^{2}\right)\right], \\
\frac{d h_{b}}{d t} & =\frac{1}{16 \pi^{2}}\left[h_{b}\left(\frac{3}{2}\left|g_{t}\right|^{2}+\frac{1}{2}\left|h_{t}\right|^{2}+\frac{9}{2}\left|h_{b}\right|^{2}+\frac{9}{2}\left|g_{b}\right|^{2}+\left|h_{\tau}\right|^{2}\right)+g_{b}\left(h_{t} g_{t}^{*}+h_{\tau} g_{\tau}^{*}\right)-\right. \\
& \left.-h_{b}\left(8 g_{3}^{2}+\frac{9}{4} g_{2}^{2}+\frac{5}{12} g_{1}^{2}\right)\right], \\
\frac{d g_{b}}{d t} & =\frac{1}{16 \pi^{2}}\left[g_{b}\left(\frac{1}{2}\left|g_{t}\right|^{2}+\frac{3}{2}\left|h_{t}\right|^{2}+\frac{9}{2}\left|h_{b}\right|^{2}+\frac{9}{2}\left|g_{b}\right|^{2}+\left|g_{\tau}\right|^{2}\right)+h_{b}\left(g_{t} h_{t}^{*}+g_{\tau} h_{\tau}^{*}\right)-\right. \\
& \left.-g_{b}\left(8 g_{3}^{2}+\frac{9}{4} g_{2}^{2}+\frac{5}{12} g_{1}^{2}\right)\right], \\
\frac{d h_{\tau}}{d t} & =\frac{1}{16 \pi^{2}}\left[h_{\tau}\left(3\left|g_{t}\right|^{2}+3\left|h_{b}\right|^{2}+\frac{5}{2}\left|h_{\tau}\right|^{2}+\frac{5}{2}\left|g_{\tau}\right|^{2}\right)+3 g_{\tau}\left(h_{b} g_{b}^{*}+h_{t} g_{t}^{*}\right)-\right. \\
& \left.-h_{\tau}\left(\frac{9}{4} g_{2}^{2}+\frac{15}{4} g_{1}^{2}\right)\right], \\
\frac{d g_{\tau}}{d t} & =\frac{1}{16 \pi^{2}}\left[g_{\tau}\left(3\left|h_{t}\right|^{2}+3\left|g_{b}\right|^{2}+\frac{5}{2}\left|h_{\tau}\right|^{2}+\frac{5}{2}\left|g_{\tau}\right|^{2}\right)+3 h_{\tau}\left(g_{b} h_{b}^{*}+g_{t} h_{t}^{*}\right)-\right. \\
& \left.-g_{\tau}\left(\frac{9}{4} g_{2}^{2}+\frac{15}{4} g_{1}^{2}\right)\right],
\end{aligned}
$$

where $t=\ln \mu$ and $\mu$ is the renormalization scale. Also the $g_{i}(\mu)$ are here the gauge couplings for the $U(1)_{Y}, S U(2)_{W}$ and $S U(3)_{C}$ interactions. 


\section{MPP conditions as an origin of the approximate custodial symmetries in the $2 \mathrm{HDM}$}

\subsection{Philosophy of using MPP in 2HDM}

It is our philosophy that, unless the parameters - couplings and masses - are restricted by some symmetry or other principle, we expect them to be essentially random. A priori the 2HDM has only the gauge symmetries and the general Poincaré symmetries. Just imposing the approximate symmetry needed to rescue the $2 \mathrm{HDM}$ from immediate disagreement with experimental facts, such as the absence of FCNC, looks like an ad hoc invention to cure the model.

In this paper we argue that such an approximate custodial symmetry can originate from the multiple point principle (MPP). Indeed we know that the MPP requirement of many degenerate vacua immediately gets fulfilled in models which possess extra global symmetries. One of the most famous examples of a symmetry that leads to a set of degenerate vacua is supersymmetry (SUSY). In exact SUSY models there are typically many minima (often even flat directions) in the scalar potential with just zero vacuum energy density. However, in phenomenologically acceptable models based on softly broken supersymmetry, MPP conditions are realised automatically but only up to soft terms in the Lagrangian. Therefore here we shall similarly postulate "hard MPP", in which we impose the degeneracy of vacua with only a limited accuracy set by the size of the soft mass term 4 in the Lagrangian. In contrast with an exact MPP, "hard MPP" gives rise to approximate global symmetries.

As a concrete realisation of such "hard MPP", we ignore the mass terms in the potential (2) and establish a relation between the MPP and global custodial $U(1)$ symmetries within the $2 \mathrm{HDM}$ which we shall refer to as a generalised (i.e. generation dependent) Peccei-Quinn symmetry. The MPP requirement of a set of degenerate vacua at some high energy scale $\Lambda$ leads to a spontaneously broken global $U(1)$ custodial symmetry. Then we take into account the contribution of mass terms in the potential (2) and allow vacua to be approximately degenerate at the MPP scale. This gives rise to a set of custodial symmetry violating couplings. These couplings allows to avoid any problems related with the presence of Nambu-Goldstone bosons in the particle spectrum that are usually unacceptable phenomenologically.

\footnotetext{
${ }^{4}$ It should be noted here that in practice we also neglected the soft Higgs mass term in the vacuum degeneracy condition used in the application of MPP to the SM [18].
} 


\subsection{Symmetry derivations from MPP in the leading approxima- tion}

Now, we aim to specify the largest possible set of global minima of the 2HDM scalar potential with almost vanishing energy density, which may exist at the MPP high energy scale $\Lambda$ where the mass terms in the potential (2) can be neglected. The most general vacuum configuration takes the form:

$$
<H_{1}>=\Phi_{1}\left(\begin{array}{l}
0 \\
1
\end{array}\right), \quad<H_{2}>=\Phi_{2}\left(\begin{array}{c}
\sin \theta \\
\cos \theta e^{i \omega}
\end{array}\right),
$$

where $\Phi_{1}^{2}+\Phi_{2}^{2}=\Lambda^{2}$. Here, the gauge is fixed so that only the real part of the lower component of $H_{1}$ gets a vacuum expectation value.

Let us assume that the 2HDM scalar potential (2) possesses a set of vacua in which the energy density goes to zero for all possible values of the phase $\omega$. The degeneracy of the vacuum configuration (6) with respect to $\omega$ implies that $\cos \theta, \Phi_{1}$ and $\Phi_{2}$ gain non-zero values at the corresponding minima. It also requires that the $2 \mathrm{HDM}$ scalar potential and all its partial derivatives are independent of $\omega$ at the MPP scale, i.e.

$$
\begin{gathered}
V_{\omega}=\frac{\lambda_{5}(\Phi)}{2} \Phi_{1}^{2} \Phi_{2}^{2} \cos ^{2} \theta e^{2 i \omega}+\left[\lambda_{6}(\Phi) \Phi_{1}^{3} \Phi_{2}+\lambda_{7}(\Phi) \Phi_{1} \Phi_{2}^{3}\right] \cos \theta e^{i \omega}+h . c .=0 \\
\frac{\partial V_{\omega}}{\partial \Phi_{1}}=\left[\lambda_{5}(\Phi) \Phi_{1} \Phi_{2}^{2}+\beta_{\lambda_{5}}(\Phi) \frac{\Phi_{1}^{3} \Phi_{2}^{2}}{2 \Phi^{2}}\right] \cos ^{2} \theta e^{2 i \omega}+ \\
\quad\left[3 \lambda_{6}(\Phi) \Phi_{1}^{2} \Phi_{2}+\beta_{\lambda_{6}}(\Phi) \frac{\Phi_{1}^{4} \Phi_{2}}{\Phi^{2}}+\lambda_{7}(\Phi) \Phi_{2}^{3}+\beta_{\lambda_{7}}(\Phi) \frac{\Phi_{1}^{2} \Phi_{2}^{3}}{\Phi^{2}}\right] \cos \theta e^{i \omega}+h .\left.c\right|_{\Phi=\Lambda}=0 \\
\frac{\partial V_{\omega}}{\partial \Phi_{2}}=\left[\lambda_{5}(\Phi) \Phi_{1}^{2} \Phi_{2}+\beta_{\lambda_{5}}(\Phi) \frac{\Phi_{1}^{2} \Phi_{2}^{3}}{2 \Phi^{2}}\right] \cos ^{2} \theta e^{2 i \omega}+ \\
{\left[\lambda_{6}(\Phi) \Phi_{1}^{3}+\beta_{\lambda_{6}}(\Phi) \frac{\Phi_{1}^{3} \Phi_{2}^{2}}{\Phi^{2}}+3 \lambda_{7}(\Phi) \Phi_{2}^{2} \Phi_{1}+\beta_{\lambda_{7}}(\Phi) \frac{\Phi_{1} \Phi_{2}^{4}}{\Phi^{2}}\right] \cos \theta e^{i \omega}+h . c .\left.\right|_{\Phi=\Lambda}=0 .}
\end{gathered}
$$

Here $\beta_{\lambda_{i}}(\Phi)=\frac{d \lambda_{i}(\Phi)}{d \ln \Phi}$ is the renormalisation group beta function for the Higgs selfcoupling $\lambda_{i}(\Phi)$. It is readily verified that the vanishing of the coefficients of $e^{i \omega}$ and $e^{2 i \omega}$ in Eqs. (7) - (9) leads to the conditions:

$$
\lambda_{5}(\Lambda)=\lambda_{6}(\Lambda)=\lambda_{7}(\Lambda)=0, \quad \beta_{\lambda_{5}}(\Lambda)=\beta_{\lambda_{6}} \Phi_{1}^{2}+\beta_{\lambda_{7}} \Phi_{2}^{2}=0 .
$$

Taking into account the derived MPP conditions (10) and substituting the vacuum configuration (6) into the quartic part of the $2 \mathrm{HDM}$ potential, one finds for any $\Phi \simeq \Lambda$ :

$$
\begin{aligned}
V\left(H_{1}, H_{2}\right) \approx & \frac{1}{2}\left(\sqrt{\lambda_{1}(\Phi)} \Phi_{1}^{2}-\sqrt{\lambda_{2}(\Phi)} \Phi_{2}^{2}\right)^{2}+ \\
& +\left(\sqrt{\lambda_{1}(\Phi) \lambda_{2}(\Phi)}+\lambda_{3}(\Phi)+\lambda_{4}(\Phi) \cos ^{2} \theta\right) \Phi_{1}^{2} \Phi_{2}^{2} .
\end{aligned}
$$


The Higgs scalar potential (11) attains its minimal value for $\cos \theta=0$ if $\lambda_{4}(\Lambda)>0$ or $\cos \theta= \pm 1$ when $\lambda_{4}(\Lambda)<0$. Since the degeneracy of the vacuum configuration (6) with respect to $\omega$ may be realised only if $\cos \theta$ has a non-zero value, the self-consistent implementation of the MPP requires $\lambda_{4}(\Lambda)$ to be negative. Then around the minimum the scalar potential can be written as

$$
V\left(H_{1}, H_{2}\right) \approx \frac{1}{2}\left(\sqrt{\lambda_{1}(\Phi)} \Phi_{1}^{2}-\sqrt{\lambda_{2}(\Phi)} \Phi_{2}^{2}\right)^{2}+\tilde{\lambda}(\Phi) \Phi_{1}^{2} \Phi_{2}^{2},
$$

where $\tilde{\lambda}=\sqrt{\lambda_{1} \lambda_{2}}+\lambda_{3}+\lambda_{4}$. If near the MPP scale the combination of the Higgs selfcouplings $\tilde{\lambda}(\Phi)$ is less than zero, then there exists a minimum with huge and negative energy density that hampers the MPP implementation. Otherwise when $\tilde{\lambda}(\Phi)>0$ the Higgs potential (12) is always positive definite, which spoils the consistent implementation of the MPP as well. Thus, in order to get a set of degenerate vacua in which the energy density tends to zero for all possible values of the phase $\omega$ at the MPP scale, one has to assume that $\tilde{\lambda}(\Lambda)=0$. Then $V\left(H_{1}, H_{2}\right)$ reaches a minimal value at

$$
\Phi_{1}=\Lambda \cos \gamma, \quad \Phi_{2}=\Lambda \sin \gamma, \quad \tan \gamma=\left(\frac{\lambda_{1}}{\lambda_{2}}\right)^{1 / 4}
$$

Next we should also require the vanishing of partial derivatives of the scalar potential (12) with respect to $\Phi_{1}$ and $\Phi_{2} 5$. This results in another MPP condition:

$$
\beta_{\tilde{\lambda}}(\Lambda)=\frac{1}{2} \beta_{\lambda_{1}}(\Lambda) \sqrt{\frac{\lambda_{2}(\Lambda)}{\lambda_{1}(\Lambda)}}+\frac{1}{2} \beta_{\lambda_{2}}(\Lambda) \sqrt{\frac{\lambda_{1}(\Lambda)}{\lambda_{2}(\Lambda)}}+\beta_{\lambda_{3}}(\Lambda)+\beta_{\lambda_{4}}(\Lambda)=0 .
$$

The MPP conditions mentioned above give rise to the following set of MPP scale vacua

$$
<H_{1}>=\left(\begin{array}{c}
0 \\
\Phi_{1}
\end{array}\right), \quad<H_{2}>=\left(\begin{array}{c}
0 \\
\Phi_{2} e^{i \omega}
\end{array}\right)
$$

which have zero energy density for any $\omega$. The Higgs field norms $\Phi_{1}$ and $\Phi_{2}$ in Eq. (15) are defined by the equations for the extrema of the $2 \mathrm{HDM}$ potential whose solution is given by Eq. (13).

Combining Eqs. (10) and (13) and using the explicit form of the $\beta$-functions for the Higgs self-couplings given in Apendix A, one obtains two conditions that quark and lepton Yukawa couplings should obey if MPP is realised in Nature:

$$
\begin{gathered}
3 h_{b}^{2}(\Lambda) g_{b}^{* 2}(\Lambda)+h_{\tau}^{2}(\Lambda) g_{\tau}^{* 2}(\Lambda)=0 \\
3 h_{b}(\Lambda) g_{b}^{*}(\Lambda)\left[\sqrt{\lambda_{2}(\Lambda)}\left|h_{b}(\Lambda)\right|^{2}+\sqrt{\lambda_{1}(\Lambda)}\left|g_{b}(\Lambda)\right|^{2}\right]+ \\
+h_{\tau}(\Lambda) g_{\tau}^{*}(\Lambda)\left[\sqrt{\lambda_{2}(\Lambda)}\left|h_{\tau}(\Lambda)\right|^{2}+\sqrt{\lambda_{1}(\Lambda)}\left|g_{\tau}(\Lambda)\right|^{2}\right]=0
\end{gathered}
$$

\footnotetext{
${ }^{5}$ The partial derivative $\partial V / \partial \theta$ goes to zero when $\cos \theta \rightarrow \pm 1$.
} 
To simplify calculations we use here the basis in the field space where only one Higgs doublet $\mathrm{H}_{2}$ interacts with the top-quark at the scale $\Lambda$, i.e. $g_{t}(\Lambda)=0$. Conditions (16) -(17) are fulfilled simultaneously only if

$$
\begin{aligned}
& \text { (I) } \quad h_{b}(\Lambda)=h_{\tau}(\Lambda)=0 ; \quad(I I) \quad g_{b}(\Lambda)=g_{\tau}(\Lambda)=0 \\
& (I I I) \quad h_{b}(\Lambda)=g_{\tau}(\Lambda)=0 ; \quad(I V) \quad g_{b}(\Lambda)=h_{\tau}(\Lambda)=0 .
\end{aligned}
$$

The solutions $(I)-(I V)$ correspond to the 2HDM Model I and Model II Yukawa couplings and their leptonic variations. In these models the MPP conditions reduce to

$$
\left\{\begin{array}{l}
\lambda_{5}(\Lambda)=\lambda_{6}(\Lambda)=\lambda_{7}(\Lambda)=0 \\
\tilde{\lambda}(\Lambda)=\beta_{\tilde{\lambda}}(\Lambda)=0
\end{array}\right.
$$

The MPP conditions were formulated in exactly this form in [41, where the multiple point principle was applied to the Model II of the two Higgs doublet extension of the SM. It is worth noting that the relations corresponding to Eq.(19) are satisfied identically in the minimal SUSY model (MSSM) at any scale lying higher than the masses of the superparticles.

Usually the existence of a large set of degenerate vacua is associated with an enlarged global symmetry of the Lagrangian of the considered model. The 2HDM is not an exception. In all the models $(I-I V)$, the quartic part of the Higgs effective potential (2) and the Lagrangian describing the interactions of quarks and leptons with the Higgs fields (4) are invariant under $Z_{2}$ symmetry transformations at the MPP scale, which prevent the appearance of flavour changing neutral currents at the tree level. Moreover when $m_{3}^{2}, \lambda_{5}$, $\lambda_{6}$ and $\lambda_{7}$ vanish, the full Lagrangian of the $2 \mathrm{HDM}$ is invariant under the transformations of an $S U(2) \times[U(1)]^{2}$ global symmetry. The mixing term $m_{3}^{2}\left(H_{1}^{\dagger} H_{2}\right)$ in the Higgs effective potential (2), which we have neglected at the MPP scale, softly breaks the $Z_{2}$ and extra $U(1)$ (Peccei-Quinn) symmetries but it does not create new sources of CP-violation or FCNC transitions. Indeed, the renormalization group flow preserves the invariance of the quartic part of the Higgs effective potential (2), as well as the invariance of the Lagrangian of the interactions of fermions with the Higgs fields, under the transformations of the $Z_{2}$ and Peccei-Quinn symmetries. This means that, if the Peccei-Quinn symmetry violating Yukawa or Higgs couplings are set to zero (or small) at some scale $\Lambda$, they will remain zero (or small) at any scale below $\Lambda$. In the Higgs sector of the general 2HDM, only the imaginary parts of $m_{3}^{2}, \lambda_{5}, \lambda_{6}$ and $\lambda_{7}$ cause CP-non-conservation. Since MPP suppresses the Higgs self-couplings which are responsible for the violation of the $\mathrm{CP}$-invariance and the complex phase of $m_{3}^{2}$ can be easily absorbed by the appropriate redefinition of the Higgs fields, MPP protects the CP-invariance within the two Higgs doublet extension of the SM. The tree-level FCNC transitions also do not emerge after the soft breakdown of 
the $Z_{2}$ and Peccei-Quinn symmetries, simply because the structure of the interactions of the quarks and leptons with the Higgs doublets remains intact.

Of course, one can argue that we only derive the custodial $Z_{2}$ symmetry for the interactions of the quarks and leptons of the third generation, while the most stringent restrictions on the Peccei-Quinn symmetry violating Yukawa couplings come from the FCNC processes involving quarks and leptons of the first two generations. Indeed, the MPP conditions for the Yukawa couplings of the third generation obtained above (see Eq.(18) ) cannot be generalised to the three generation case in a straightforward way at leading order.

\subsection{MPP symmetry derivation to one loop accuracy}

In this subsection we shall discuss symmetry derivation from MPP to one loop accuracy and shall indeed achieve the derivation of a Peccei-Quinn-like symmetry even for the lower mass generations. In the case when three generations of quarks and leptons have nonnegligible couplings to the Higgs doublets, all the SM bosons and fermions contribute to the Higgs effective potential. It is then convenient to present the potential in the following form

$$
V_{\text {eff }}\left(H_{1}, H_{2}\right)=\sum_{n=0}^{\infty} V_{n}\left(H_{1}, H_{2}\right),
$$

where $V_{0}$ corresponds to the tree level Higgs boson potential, while $V_{n}$ represents the $n$-loop contribution to $V_{\text {eff }}$. In the one-loop approximation we have

$$
V_{1}=\frac{1}{64 \pi^{2}} \operatorname{Str}|M|^{4}\left[\log \frac{|M|^{2}}{\mu^{2}}-C\right],
$$

where $M$ is the mass matrix for the bosons and fermions in the model. Here the supertrace operator counts positively (negatively) the number of degrees of freedom for the different bosonic (fermionic) fields, $C$ is a diagonal matrix which depends on the renormalisation scheme, while $\mu$ is the renormalisation scale. Previously we restricted our consideration to the leading $\log$ approximation, i.e. we replaced $\log \frac{|M|^{2}}{\mu^{2}}$ by $\log \frac{\Phi^{2}}{\mu^{2}}$ in Eq. (21) and summed all the leading logs using the renormalisation group equations. A more accurate analysis, which we shall perform in the next paragraph, requires us to take into account all terms in Eq. (21).

The independence of the Higgs effective potential on $\omega$ at the MPP scale implies that any order partial derivatives of $V_{\text {eff }}$ with respect to $\omega$ vanish at this scale. From Eq. (21) it becomes clear that this can be achieved only when the masses of all the particles are independent of $\omega$ near the MPP scale vacuat. Near the vacuum configuration parametrized

\footnotetext{
${ }^{6}$ This is expected intuitively and can be proved formally by considering $V_{1}$ as an analytic function of $z=e^{i \omega}$, which is required to be constant for $|z|=1$
} 
by Eqs. (13) and (15), the mass terms of the quarks and leptons take the form

$$
\begin{gathered}
\mathcal{L}_{\text {mass }}=\sum_{f=u, d, l}\left(\bar{f}_{R} \bar{f}_{L}\right)\left(\begin{array}{cc}
0 & M_{f} \\
M_{f}^{\dagger} & 0
\end{array}\right)\left(\begin{array}{c}
f_{R} \\
f_{L}
\end{array}\right), \\
M_{u}=H_{u} \Phi_{2} e^{i \omega}+G_{u} \Phi_{1}, \quad M_{d}=G_{d} \Phi_{2} e^{i \omega}+H_{d} \Phi_{1}, \\
M_{l}=G_{l} \Phi_{2} e^{i \omega}+H_{l} \Phi_{1} .
\end{gathered}
$$

Here $H_{f}$ and $G_{f}$ are $3 \times 3$ matrices that replace $h_{f}$ and $g_{f}$ in the Lagrangian (4)). Instead of the mass matrices of quarks and leptons $\left(\mathcal{M}_{f}\right)$ one can consider

$$
\mathcal{M}_{f} \mathcal{M}_{f}^{\dagger}=\left(\begin{array}{cc}
M_{f} M_{f}^{\dagger} & 0 \\
0 & M_{f}^{\dagger} M_{f}
\end{array}\right),
$$

whose eigenvalues are positive definite and equal to the absolute values of the fermion masses squared. The eigenvalues of $\mathcal{M}_{f} \mathcal{M}_{f}^{\dagger}$ will not depend on $\omega$ only if

$$
G_{f}^{\dagger} H_{f}=H_{f}^{\dagger} G_{f}=G_{f} H_{f}^{\dagger}=H_{f} G_{f}^{\dagger}=0
$$

The conditions (25) can be satisfied only when either $\operatorname{det} G_{f}=0$ or $\operatorname{det} H_{f}=0$ or both determinants vanish. By means of unitary transformations of the right-handed and lefthanded states, one can easily diagonalise the Yukawa matrices $H_{f}$. Then it can be readily shown that the solutions of Eq. (25) can be written as follows:

$$
H_{f}=\left(\begin{array}{ccc}
h_{f_{1}} & 0 & 0 \\
0 & h_{f_{2}} & 0 \\
0 & 0 & h_{f_{3}}
\end{array}\right), \quad G_{f}=\left(\begin{array}{ccc}
g_{f_{1}} & 0 & 0 \\
0 & g_{f_{2}} & 0 \\
0 & 0 & g_{f_{3}}
\end{array}\right), \quad h_{f_{i}} \cdot g_{f_{i}}=0 .
$$

The solutions obtained above guarantee the suppression of FCNC processes at the MPP scale vacua. Since according to the solutions (26) either $h_{f_{i}}$ or $g_{f_{i}}$ equals zero, each fermion eigenstate couples to only one Higgs doublet (either $H_{1}$ or $H_{2}$ ) so that the conditions of the Glashow-Weinberg theorem [42] are satisfied and non-diagonal flavour transitions are forbidden at the tree level. Moreover the MPP solution for the Yukawa couplings derived above implies that the Lagrangian for the Higgs-fermion interactions is invariant under the symmetry transformations:

$$
\begin{array}{llll}
H_{1} \rightarrow e^{i \alpha} H_{1}, & u_{R_{i}}^{\prime} \rightarrow e^{i \alpha} u_{R_{i}}^{\prime}, & d_{R_{i}}^{\prime} \rightarrow e^{-i \alpha} d_{R_{i}}^{\prime}, & e_{R_{i}}^{\prime} \rightarrow e^{-i \alpha} e_{R_{i}}^{\prime}, \\
H_{2} \rightarrow e^{i \beta} H_{2}, & u_{R_{i}}^{\prime \prime} \rightarrow e^{i \beta} u_{R_{i}}^{\prime \prime}, & d_{R_{i}}^{\prime \prime} \rightarrow e^{-i \beta} d_{R_{i}}^{\prime \prime}, & e_{R_{i}}^{\prime \prime} \rightarrow e^{-i \beta} e_{R_{i}}^{\prime \prime} .
\end{array}
$$

Here $u_{R_{i}}^{\prime}, d_{R_{i}}^{\prime}, e_{R_{i}}^{\prime}$ are right-handed quarks and leptons which couple to $H_{1}$ while $u_{R_{i}}^{\prime \prime}$, $d_{R_{i}}^{\prime \prime}, e_{R_{i}}^{\prime \prime}$ are right-handed fermions that interact with $H_{2}$. These two global $U(1)$ symmetries (27) are responsible for the suppression of FCNC effects in the considered MPP 
scenarid7. Really one linear combination of these two symmetries - namely the one for which $\alpha=\beta$ - is just a symmetry inherited from the Standard Model. It just corresponds to a combination of the well-known accidental symmetries of baryon number and lepton number conservation together with the weak hypercharge gauge symmetry. The other $U(1)$ symmetry, corresponding to $\alpha=-\beta$, is a generalisation of the Peccei-Quinn chiral $U(1)$ symmetry. It is, of course, only the latter that is truly derived from MPP. It should be remarked that the $Z_{2}$ subgroup of this generalised Peccei-Quinn symmetry, obtained by setting $\alpha=\pi$ and $\beta=0$, acts as a custodial symmetry to prevent FCNC.

The renormalisation group flow of Yukawa couplings does not spoil the invariance of the Lagrangian describing the interactions of quarks and leptons with the Higgs bosons under the custodial symmetry transformations (27). As a consequence the same symmetries forbid non-diagonal flavour transitions near the electroweak scale vacuum at the tree level. Thus MPP provides a reliable mechanism for the suppression of FCNC processes. Some of the MPP solutions given by Eq. (26) are very well known. For example, when either $G_{f}$ or $H_{f}$ vanishes the suppression of non-diagonal flavour processes is caused by the usual Peccei-Quinn symmetry. In this sense the MPP solutions derived above may be considered as generalisations of the well-known Peccei-Quinn symmetric solution of the FCNC problem.

There is an important feature that may allow us to distinguish the softly broken Peccei-Quinn symmetric solution of the FCNC problem from the MPP inspired two Higgs doublet models. The point is that, in the MPP inspired two Higgs doublet extension of the SM, the Peccei-Quinn-like symmetry violating Yukawa couplings can have non-zero values. Actually, one may notice that we did not require exact degeneracy of vacua at the electroweak and MPP scale. Since we ignore all mass terms in the 2HDM potential (2) during the derivation of the MPP conditions, the energy density of the vacua at the scale $\Lambda$ is expected to be of the order of $v^{2} \Lambda^{2}$ while the total vacuum energy density at the physical vacuum is set by $v^{4}$. Thus MPP here postulates the degeneracy of all vacua with the accuracy $v^{2} \Lambda^{2}$. It means that the Higgs self-couplings $\lambda_{5,6,7}(\Lambda)$ which break the Peccei-Quinn-like symmetry may take on small but non-zero values, i.e.

$$
\left|\lambda_{5}(\Lambda)\right|,\left|\lambda_{6}(\Lambda)\right|,\left|\lambda_{7}(\Lambda)\right| \lesssim \frac{v^{2}}{\Lambda^{2}}
$$

Because of this the $\beta$-functions $\beta_{\lambda_{5}}(\Lambda)$ and $\beta_{\lambda_{6}}(\Lambda) \Phi_{1}^{2}+\beta_{\lambda_{7}}(\Lambda) \Phi_{2}^{2}$ appearing in Eqs. (8) $-(9)$ do not vanish exactly as well. This permits us to establish constraints on the values of

\footnotetext{
7 In principle we should have included a kinetic mixing term $\varkappa\left[\left(D_{\mu} H_{1}\right)^{\dagger}\left(D_{\mu} H_{2}\right)+\right.$ h.c. $]$ in the Lagrangian. However one can show that the existence of a set of degenerate vacua with respect to $\omega$ implies that $\varkappa=0$ at the scale $\Lambda$. Nevertheless small custodial symmetry violating couplings would induce a small non-zero value of $\varkappa$
} 
the Peccei-Quinn-like symmetry violating Yukawa couplings

$$
\begin{gathered}
\beta_{\lambda_{5}}(\Lambda) \simeq \frac{h^{2}(\Lambda) g^{2}(\Lambda)}{(4 \pi)^{2}} \lesssim v^{2} / \Lambda^{2} \\
\beta_{\lambda_{6}}(\Lambda) \cos ^{2} \gamma+\beta_{\lambda_{7}}(\Lambda) \sin ^{2} \gamma \simeq \frac{h^{3}(\Lambda) g(\Lambda)}{(4 \pi)^{2}} \lesssim v^{2} / \Lambda^{2}
\end{gathered}
$$

where $h$ should be associated with the Yukawa couplings which preserve the PecceiQuinn-like symmetry, while $g$ is a typical value of the Peccei-Quinn-like symmetry violating Yukawa couplings. Here it is worth emphasizing that any Yukawa coupling which breaks the Peccei-Quinn-like symmetry will contribute to the left-hand side of Eqs. (29). Therefore the inequalities (29) constrain all Peccei-Quinn-like symmetry violating Yukawa couplings, including the ones which induce FCNC transitions of quarks and leptons of the first two generations.

If $\Lambda$ is quite close to the Planck scale then all couplings that break Peccei-Quinn-like or custodial $Z_{2}$ symmetries are really tiny: $\lambda_{5,6,7} \lesssim 10^{-34}$ and $g \lesssim 10^{-32} / h^{3}$. When $h$ varies from 1 to $10^{-5}$, the limits on the Peccei-Quinn-like symmetry violating Yukawa couplings change from $10^{-32}$ to $10^{-17}$. For such tiny values of $\lambda_{5,6,7}$ and $g$, all $\mathrm{CP}$-violation and FCNC effects are extremely strongly suppressed and do not lead to any phenomena which could be observed in the near future.

However, in order to get suitable suppression of the FCNC transitions observed experimentally, the MPP scale does not necessarily have to be as large as the Planck scale. For instance, at very large values of $\tan \beta$ in model II of the $2 \mathrm{HDM}$, when $h_{b} \sim h_{\tau} \sim 1$, the appropriate suppression of the Peccei-Quinn-like symmetry violating Yukawa couplings in $K$ meson physics may be obtained even for $\Lambda \simeq 100-1000 \mathrm{TeV}$, if we assume that all the Peccei-Quinn-like symmetry violating couplings are of the same order of magnitude or that their pattern exhibits a hierarchical structure similar to the one suggested by Cheng and Sher [56].

If the $b$-quark and $\tau$ lepton Yukawa couplings are as small as in the SM, i.e. $h \sim 10^{-2}$, then to ensure the absence of large FCNC transitions the MPP scale should be pushed up to $10^{5}-10^{6} \mathrm{TeV}$. The contribution of non-renormalisable terms, arising from new physics at such high scales $\Lambda$, to FCNC processes is also negligibly small. In this case $\left|\lambda_{5,6,7}\right| \lesssim 10^{-12}-10^{-14}$.

\section{The running of the Yukawa couplings and the quasi-fixed point solutions}

Now we consider the RG flow of the Yukawa couplings within the MPP inspired 2HDM with approximate generalised Peccei-Quinn and $Z_{2}$ symmetries. When the MPP scale 
is relatively high the Peccei-Quinn-like symmetry violating Yukawa and Higgs couplings are extremely small, which allows us to suppress non-diagonal flavour transitions and $\mathrm{CP}$-violating interactions. Meanwhile, if the interval between the MPP and electroweak scales is large enough, the solutions of the RG equations are concentrated in the vicinity of the quasi-fixed points. The quasi-fixed point scenario in the $2 \mathrm{HDM}$ was analysed in [43], [57]-62].

\subsection{Quasi-fixed point scenario at moderate $\tan \beta$}

Because the Yukawa couplings of quarks and leptons of the first two generation, as well as the Peccei-Quinn-like symmetry violating Yukawa and Higgs couplings, are negligibly small they are irrelevant for our analysis of RG flow. So we return back to the four MPP solutions (18) derived in the previous section. Moreover at moderate values of $\tan \beta$ ( $\tan \beta \lesssim 10$ ), the Yukawa couplings of the $b$-quark and $\tau$-lepton are also very small and can be safely ignored. As a result the renormalisation group equations (5) are simplified drastically and an exact analytic solution for the top quark Yukawa coupling may be obtained. It can be written as follows:

$$
\begin{gathered}
Y_{t}(\mu)=\frac{\frac{2 E(l)}{9 F(l)}}{1+\frac{2}{9 Y_{t}(\Lambda) F(l)}}, \quad \tilde{\alpha}_{i}(\mu)=\frac{\tilde{\alpha}_{i}(\Lambda)}{1+b_{i} \tilde{\alpha}_{i}(\Lambda) l}, \\
E(l)=\left[\frac{\tilde{\alpha}_{3}(\mu)}{\tilde{\alpha}_{3}(\Lambda)}\right]^{8 / 7}\left[\frac{\tilde{\alpha}_{2}(\mu)}{\tilde{\alpha}_{2}(\Lambda)}\right]^{3 / 4}\left[\frac{\tilde{\alpha}_{1}(\mu)}{\tilde{\alpha}_{1}(\Lambda)}\right]^{-17 / 84}, \quad F(l)=\int_{0}^{l} E\left(l^{\prime}\right) d l^{\prime},
\end{gathered}
$$

where the index $i$ varies from 1 to $3, b_{1}=7, b_{2}=-3, b_{3}=-7, l=\ln \left(\Lambda^{2} / \mu^{2}\right)$, $\tilde{\alpha}_{i}(\mu)=\left(\frac{g_{i}(\mu)}{4 \pi}\right)^{2}, Y_{t}(\mu)=\left(\frac{h_{t}(\mu)}{4 \pi}\right)^{2}$. If the MPP scale is very high and $h_{t}^{2}(\Lambda) \gtrsim 1$, the second term in the denominator of the expression describing the evolution of $Y_{t}(\mu)$ is much smaller than unity at the electroweak scale. For example, when $\Lambda$ is of the order of the Planck scale and $l=l_{0}=\ln \left(\Lambda^{2} / M_{t}^{2}\right)$, the term $\frac{2}{9 Y_{t}(\Lambda) F\left(l_{0}\right)}$ is approximately equal to $\frac{1}{7 h_{t}^{2}(\Lambda)}$. Due to the small numerical coefficient in front of $1 / h_{t}^{2}(\Lambda)$, the dependence of $h_{t}^{2}(\mu)$ on its initial value $h_{t}^{2}(\Lambda)$ disappears when $h_{t}^{2}(\Lambda) \gtrsim 1$ and all solutions of the $\mathrm{RG}$ equation for the top quark Yukawa coupling are concentrated in a narrow interval near the quasi-fixed point [43], 63]:

$$
Y_{\mathrm{QFP}}\left(M_{t}\right)=\frac{2 E\left(l_{0}\right)}{9 F\left(l_{0}\right)}
$$

Formally a solution of this type can be obtained in the limit when $Y_{t}(\Lambda)$ is infinitely large. But in reality the convergence of RG solutions to the quasi-fixed point (31) does not 
require extremely large values of the top quark Yukawa coupling at the MPP scale, if $\Lambda$ is high enough.

In Fig. 1a we examine the deviations of the solutions of the RG equations from the quasi-fixed point (31) at the electroweak scale as a function of the MPP scale. The dashdotted, solid and dashed curves represent the quasifixed point solution $\left(h_{t}(\Lambda)>>1\right)$ and the solutions to the RG equations that correspond to $h_{t}^{2}(\Lambda)=10$ and $h_{t}^{2}(\Lambda)=2.25$. The dash-dotted, solid and dashed lines are rather close to each other at large values of $\Lambda$ $\left(\Lambda \gtrsim 10^{13} \mathrm{GeV}\right)$. This demonstrates that the solutions of the RG equation for $h_{t}(\mu)$ are attracted towards the quasi-fixed point relatively strongly. At low values of the MPP scale, $\Lambda \simeq 10^{4}-10^{7} \mathrm{GeV}$, the convergence of $h_{t}(\mu)$ to the quasi-fixed point is quite weak, so that it is rather difficult to get a reasonable prediction for the top quark Yukawa coupling at the electroweak scale. Generally the quasi-fixed point solution provides an upper bound on $h_{t}\left(M_{t}\right)$.

The convergence of the RG solutions to the quasi-fixed point allows us to predict the value of the top quark Yukawa coupling at the electroweak scale for each fixed value of the MPP scale. Then, using the relation between the running mass and Yukawa coupling of the $t$-quark

$$
m_{t}\left(M_{t}\right)=\frac{h_{t}\left(M_{t}\right)}{\sqrt{2}} v \sin \beta,
$$

one can find the value of $\tan \beta$ that corresponds to the quasi-fixed point (31). Here we use the world average mass of the top quark $M_{t}=171.4 \pm 2.1 \mathrm{GeV}$ (see [35]) and the relationship between the $t$-quark pole $\left(M_{t}\right)$ and running $\left(m_{t}(\mu)\right)$ masses [64]-68],

$$
m_{t}\left(M_{t}\right)=M_{t}\left[1-1.333 \frac{\alpha_{s}\left(M_{t}\right)}{\pi}-9.125\left(\frac{\alpha_{s}\left(M_{t}\right)}{\pi}\right)^{2}\right] .
$$

We find that, in the two-loop approximation, $m_{t}\left(M_{t}\right) \simeq 161.6 \pm 2 \mathrm{GeV}$.

The results of our calculations are summarised in Fig. 1b, where we set $m_{t}\left(M_{t}\right) \simeq 161.6 \mathrm{GeV}$. In Fig. $1 \mathrm{~b}$ we plot the values of $\tan \beta$ that correspond to $h_{t}^{2}(\Lambda)>>1, h_{t}^{2}(\Lambda)=10$ and $h_{t}^{2}(\Lambda)=2.25$ (dash-dotted, solid and dashed lines respectively) as a function of the scale $\Lambda$. From this figure it becomes clear that, at large values of $h_{t}(\Lambda) \gtrsim 1.5$, the RG solutions for the top quark Yukawa coupling are gathered in the vicinity of $\tan \beta=1$ at the electroweak scale. The dash-dotted curve in Fig. 1(b) corresponds to the maximal possible value of $h_{t}\left(M_{t}\right)$ and, as a consequence, represents the lower bound on $\tan \beta$.

\subsection{Quasi-fixed point solutions at large $\tan \beta$}

When the values of $\tan \beta$ are large the solutions of the $\mathrm{RG}$ equations are also focused near quasi-fixed points, if the appropriate Yukawa couplings at the MPP scale are relatively 
large. But in this case the position of the quasi-fixed point is model dependent. For example, in the model $(I)$ (see Eq.(18) $) g_{b}(\Lambda)$ and $g_{\tau}(\Lambda)$ cannot be large, because this results in large masses for the $b$-quark and $\tau$-lepton $\sim 100 \mathrm{GeV}$. Therefore, in model $(I)$ of the MPP inspired 2HDM, there is only one phenomenologically acceptable quasi-fixed point solution which is given by Eq. (31).

In the case of the MPP solution $(I V)$ both $h_{t}(\Lambda)$ and $h_{b}(\Lambda)$ are allowed to be large, because the masses of the top and bottom quarks are generated by two different Higgs doublets whose vacuum expectation values can be very different and be used to induce a large hierarchy between $m_{t}\left(M_{t}\right)$ and $m_{b}\left(M_{t}\right)$. To ensure that $m_{t}\left(M_{t}\right)>>m_{\tau}\left(M_{t}\right)$ in the considered model, the $\tau$-lepton Yukawa coupling has to be always much smaller than the top quark one. Therefore we can neglect $g_{\tau}$ in our analysis of the RG flow. Then the two remaining RG equations, which describe the evolution of $h_{t}(\mu)$ and $h_{b}(\mu)$, are invariant under the interchange $h_{t}(\mu) \leftrightarrow h_{b}(\mu)$, if we ignore the $U(1)_{Y}$ gauge coupling which does not much affect the running of the Yukawa couplings. In the limit when $g_{1} \rightarrow 0$ and $Y_{t}(\Lambda)=Y_{b}(\Lambda)=Y_{0}$, the solutions of the RG equations take the form

$$
\begin{gathered}
Y_{t}(\mu) \simeq Y_{b}(\mu) \simeq \frac{\frac{E_{1}(l)}{5 F_{1}(l)}}{1+\frac{1}{5 Y_{0} F_{1}(l)}}, \\
E_{1}(l)=\left[\frac{\tilde{\alpha}_{3}(\mu)}{\tilde{\alpha}_{3}(\Lambda)}\right]^{8 / 7}\left[\frac{\tilde{\alpha}_{2}(\mu)}{\tilde{\alpha}_{2}(\Lambda)}\right]^{3 / 4}, \quad F_{1}(l)=\int_{0}^{l} E_{1}\left(l^{\prime}\right) d l^{\prime},
\end{gathered}
$$

where $Y_{b}(\mu)=\left(\frac{h_{b}(\mu)}{4 \pi}\right)^{2}$. Eq. (34) demonstrates that, at large values of $Y_{0}$, the solutions of the RG equations for $Y_{t}(\mu)$ and $Y_{b}(\mu)$ approach the quasi-fixed point at the electroweak scale:

$$
Y_{t}\left(M_{t}\right) \simeq Y_{b}\left(M_{t}\right) \simeq \frac{E_{1}\left(l_{0}\right)}{5 F_{1}\left(l_{0}\right)} .
$$

Substituting the obtained prediction for the $b$-quark Yukawa coupling into the equation

$$
m_{b}\left(M_{t}\right)=\frac{h_{b}\left(M_{t}\right)}{\sqrt{2}} v \cos \beta
$$

which relates the running $b$-quark mass $m_{b}\left(M_{t}\right)$ with its Yukawa coupling at the electroweak scale, one can determine the value of $\tan \beta$ that corresponds to the quasi-fixed point solution (35). Because $h_{t}\left(M_{t}\right) \simeq h_{b}\left(M_{t}\right)$, one finds that $\tan \beta \simeq m_{t}\left(M_{t}\right) / m_{b}\left(M_{t}\right)=55-60$. For such large values of $\tan \beta$ the running mass of the $t$-quark does not depend on $\tan \beta$, i.e. $m_{t}\left(M_{t}\right) \approx \frac{h_{t}\left(M_{t}\right)}{\sqrt{2}} v$, and can also be predicted. The results of our numerical computations are presented in Table 1. From Table 
1 it is obvious that the quasi-fixed point solution (35) for the MPP solution (IV) results in an unacceptably large value for the running top quark mass.

Another quasi-fixed point arises in the framework of the MPP solution (III) (see Eq. (18)). In this case the non-zero $b$-quark Yukawa coupling $g_{b}$ has to be negligibly small to guarantee that $m_{b}\left(M_{t}\right)<<m_{t}\left(M_{t}\right)$. Nevertheless the $\tau$-lepton Yukawa coupling may be comparable with the top quark one at the MPP scale, since the masses of the $t$-quark and $\tau$-lepton are induced by different Higgs doublets. In the limit when the $b$-quark Yukawa coupling goes to zero, the RG flow of $h_{t}(\mu)$ and $h_{\tau}(\mu)$ is described by two independent first order differential equations which can be solved analytically. The corresponding analytic solution for the top quark Yukawa coupling is given by Eq. (30), while the solution for the $\tau$-lepton one can be written in the following form:

$$
\begin{gathered}
Y_{\tau}(\mu)=\frac{\frac{2 E_{2}(l)}{5 F_{2}(l)}}{1+\frac{2}{5 Y_{\tau}(\Lambda) F_{2}(l)}}, \\
E_{2}(l)=\left[\frac{\tilde{\alpha}_{2}(\mu)}{\tilde{\alpha}_{2}(\Lambda)}\right]^{3 / 4}\left[\frac{\tilde{\alpha}_{1}(\mu)}{\tilde{\alpha}_{1}(\Lambda)}\right]^{-15 / 28}, \quad F_{2}(l)=\int_{0}^{l} E_{2}\left(l^{\prime}\right) d l^{\prime},
\end{gathered}
$$

where $Y_{\tau}(\mu)=\left(\frac{h_{\tau}(\mu)}{4 \pi}\right)^{2}$.

In the $\left(\rho_{t}, \rho_{\tau}\right)$ plane, where $\rho_{t}(\mu)=Y_{t}(\mu) / \tilde{\alpha}_{3}(\mu)=\left(h_{t}(\mu) / g_{3}(\mu)\right)^{2}$ and $\rho_{\tau}(\mu)=Y_{\tau}(\mu) / \tilde{\alpha}_{3}(\mu)=\left(h_{\tau}(\mu) / g_{3}(\mu)\right)^{2}$, the allowed part of the parameter space at the electroweak scale is limited by two perpendicular lines

$$
\rho_{t}=\frac{2 E\left(l_{0}\right)}{9 \tilde{\alpha}_{3}\left(M_{t}\right) F\left(l_{0}\right)}, \quad \quad \rho_{\tau}=\frac{2 E_{2}\left(l_{0}\right)}{5 \tilde{\alpha}_{3}\left(M_{t}\right) F_{2}\left(l_{0}\right)},
$$

where $l_{0}=\ln \left(\Lambda^{2} / M_{t}^{2}\right)$. The two lines (38) together form a quasi-fixed (or Hill type effective) line. The solutions of the RG equations (30) and (37) are gathered near this line, when the Yukawa couplings at the MPP scale increase. At the same time if $l /(4 \pi) \gtrsim 1$, $\rho_{t}(\mu)$ and $\rho_{\tau}(\mu)$ are attracted towards the invariant line, which can be parametrised as:

$$
\left\{\begin{aligned}
\rho_{t}(\mu) & =\frac{2 E(l)}{9 \tilde{\alpha}_{3}(\mu) F(l)} \\
\rho_{\tau}(\mu) & =\frac{2 E_{2}(l)}{5 \tilde{\alpha}_{3}(\mu) F_{2}(l)} .
\end{aligned}\right.
$$

Infrared fixed lines and surfaces, as well as their properties, were studied in detail in [69][71]. When $l=\ln \left(\Lambda^{2} / \mu^{2}\right)$ goes to zero, the invariant line (39) approaches its asymptotic limit where $\rho_{t}, \rho_{\tau}>>1$ and $\rho_{\tau} \rightarrow 1.8 \rho_{t}$, which is a fixed point of the RG equations for the Yukawa couplings in the gaugeless limit $\left(g_{1}=g_{2}=g_{3}=0\right)$. The invariant line connects 
this fixed point with the infrared stable fixed point $\left(\frac{2}{9}, 0\right)$ to which all solutions converge when either $l \rightarrow \infty$ or $g_{3}(\mu)$ approaches a Landau pole. At the electroweak scale $\rho_{t}(\mu)$ and $\rho_{\tau}(\mu)$ are concentrated near the quasi-fixed point $\left(\frac{2 E\left(l_{0}\right)}{9 \tilde{\alpha}_{3}\left(M_{t}\right) F\left(l_{0}\right)}, \frac{2 E_{2}\left(l_{0}\right)}{5 \tilde{\alpha}_{3}\left(M_{t}\right) F_{2}\left(l_{0}\right)}\right)$, which coincides with the intersection point of the invariant and Hill type effective lines [72]-74].

The value of $\tan \beta$ at which this quasi-fixed point solution is realised can be found from the relation between the running mass and the Yukawa coupling of the $\tau$-lepton:

$$
m_{\tau}\left(M_{t}\right)=\frac{h_{\tau}\left(M_{t}\right)}{\sqrt{2}} v \cos \beta .
$$

Eq. (40) results in extremely large values of $\tan \beta=90-100$. At these values of $\tan \beta$, the running mass of the $t$-quark is set by $h_{t}\left(M_{t}\right)$ alone. This permits us to evaluate $m_{t}\left(M_{t}\right)$ in the vicinity of the considered quasi-fixed point. However the prediction obtained for $m_{t}\left(M_{t}\right)$ is considerably higher than the experimental running mass of the $t$-quark calculated by means of Eq. (33) (see Table 1).

The method of computation of the quasi-fixed point coordinates discussed above can be applied to the determination of the position of the quasi-fixed point in the MPP scenario $(I V)$ as well. Once again the Hill type effective line restricts the allowed range of the parameter space in the $\left(\rho_{t}, \rho_{b}\right)$ plane at the electroweak scale. Here $\rho_{b}(\mu)=Y_{b}(\mu) / \tilde{\alpha}_{3}(\mu)=\left(h_{b}(\mu) / g_{3}(\mu)\right)^{2}$. Outside this range the solutions of the renormalisation group equations for $h_{t}(t)$ and $h_{b}(t)$ develop a Landau pole below the scale $\Lambda$. The quasi-fixed point in this model appears as a result of the intersection of the Hill type effective line and the invariant line, which connects a fixed point of the RG equations in the gaugeless limit $\left(\rho_{t}=\rho_{b}\right)$ with the infrared stable fixed point $\left(\rho_{t}=\rho_{b}=1 / 5\right)[72]$-[74].

The most difficult case for the analysis of RG flow is the MPP solution $(I I)$ where $h_{t}(\Lambda), h_{b}(\Lambda)$ and $h_{\tau}(\Lambda)$ can be large simultaneously, while the mass hierarchy within the third generation of fermions is caused by large values of $\tan \beta$. In the model $(I I)$, for each allowed set of top quark and $b$-quark Yukawa couplings at the electroweak scale, the interval of variation of $h_{\tau}\left(M_{t}\right)$ is limited from above. This theoretical restriction comes from the requirement of the validity of perturbation theory up to the MPP scale. A change of $h_{t}\left(M_{t}\right)$ and $h_{b}\left(M_{t}\right)$ leads either to a growth or a reduction in the upper limit on $h_{\tau}\left(M_{t}\right)$. As a result, at the electroweak scale, the allowed range of Yukawa couplings in the $\left(\rho_{t}, \rho_{b}, \rho_{\tau}\right)$ space is limited by a Hill type effective surface. With increasing $h_{t}(\Lambda)$, $h_{b}(\Lambda)$ and $h_{\tau}(\Lambda)$, the solutions of the RG equations are gathered near this surface. At the same time if the interval of evolution is relatively large, i.e. $l_{0}=\ln \frac{\Lambda^{2}}{M_{t}^{2}} \gtrsim 4 \pi$, the solutions of the RG equations for $h_{t}(\mu), h_{b}(\mu)$ and $h_{\tau}(\mu)$ are also attracted to the invariant line, which joins together a fixed point of the $\mathrm{RG}$ equations in the gaugeless 
$\operatorname{limit}\left(\rho_{b}=\frac{11}{15} \rho_{t}, \rho_{\tau}=\frac{16}{15} \rho_{t}\right)$ and an infrared stable fixed point $\left(\rho_{t}=\rho_{b}=1 / 5, \rho_{\tau}=0\right)$ [69]-[71]. Thus, at the electroweak scale, the solutions of the RG equations for the Yukawa couplings are concentrated near the quasi-fixed point, which is located at the intersection of the invariant line with the Hill type effective surface [72]-[74].

The values of $\rho_{t}\left(M_{t}\right), \rho_{b}\left(M_{t}\right)$ and $\rho_{\tau}\left(M_{t}\right)$ that correspond to this quasi-fixed point are given in Table 1. As before, using the relation between the running $\tau$-lepton mass and $h_{\tau}\left(M_{t}\right)$ (40), one can find $\tan \beta$. Substituting the obtained value of $\tan \beta$ into Eqs. (32) and (36), the running quark masses $m_{t}\left(M_{t}\right)$ and $m_{b}\left(M_{t}\right)$ can be predicted anew. From Table 1 it becomes clear that the running top quark mass is still unacceptably large, while the prediction for $m_{b}\left(M_{t}\right)$ is too small compared to the experimental value [75] of $m_{b}\left(M_{t}\right)=2.75 \pm 0.09 \mathrm{GeV}$. As a consequence we conclude that, in the MPP scenarios considered above, it is rather difficult to get a self-consistent solution if two or three Yukawa couplings are greater than unity at the scale $\Lambda$, because in the dominant part of parameter space $m_{t}\left(M_{t}\right)$ tends to be significantly higher than $160-170 \mathrm{GeV}$.

\section{Higgs phenomenology}

\subsection{The RG flow of the Higgs self-couplings near the quasi-fixed point}

Nevertheless a self-consistent solution can be obtained in the case when only $h_{t}(\Lambda) \gtrsim 1$, while all other Yukawa couplings are small. In this limit only the top quark Yukawa coupling is relevant and the solutions of the $\mathrm{RG}$ equations for $h_{t}(\mu)$ are attracted to the quasi-fixed point (31). With increasing $h_{t}(\Lambda)$, the solutions of the RG equations for the Higgs self-couplings are also gathered near the quasi-fixed points. To establish the positions of the quasi-fixed points for $\lambda_{i}(\mu)$, we apply the method of determination developed in Section 4. For the purposes of our RG studies, it is convenient to introduce

$$
\rho_{i}(\mu)=\frac{\lambda_{i}(\mu)}{g_{3}^{2}(\mu)}, \quad R_{i}(\mu)=\frac{\rho_{i}(\mu)}{\rho_{t}(\mu)}=\frac{\lambda_{i}(\mu)}{h_{t}^{2}(\mu)}
$$

where the index $i$ runs from 1 to 4 . When $\lambda_{1}(\Lambda), \lambda_{2}(\Lambda), \lambda_{3}(\Lambda), \lambda_{4}(\Lambda)$ and the top quark Yukawa coupling at the MPP scale grow, the corresponding solutions of the RG equations are focused near the intersection point of the invariant line and the Hill type effective surface that sets an upper limit on the values of $\rho_{1}, \rho_{2}, \rho_{3}$ and $\rho_{4}$ at the electroweak scale (see [72]-[74]).

As was revealed in the previous subsection, the invariant line connects a stable fixed point of the $\mathrm{RG}$ equations in the gaugeless limit with an infrared fixed point. When the 
strong gauge coupling approaches a Landau pole, all solutions of the RG equations are concentrated near the infrared fixed point

$$
\rho_{t}=\frac{2}{9}, \quad \rho_{1}=0, \quad \rho_{2}=\frac{\sqrt{689}-25}{36} \simeq 0.0347, \quad \rho_{3}=0, \quad \rho_{4}=0 .
$$

This is the only stable fixed point in the infrared region. In the gaugeless limit fixed points obey the following system of nonlinear algebraic equations:

$$
\left\{\begin{array}{l}
12 R_{1}^{2}+4 R_{3}^{2}+4 R_{3} R_{4}+2 R_{4}^{2}-9 R_{1}=0 \\
12 R_{2}^{2}+4 R_{3}^{2}+4 R_{3} R_{4}+2 R_{4}^{2}+3 R_{2}-12=0 \\
2\left(R_{1}+R_{2}\right)\left(3 R_{3}+R_{4}\right)+4 R_{3}^{2}+2 R_{4}^{2}-3 R_{3}=0 \\
2 R_{4}\left(R_{1}+R_{2}+4 R_{3}+2 R_{4}\right)-3 R_{4}=0 .
\end{array}\right.
$$

The equations (43) come from the requirement that the beta-functions of the $R_{i}$ vanish in the limit $g_{i} \rightarrow 0$. The numerical solutions $R_{i}^{0}$ of Eqs. (43) are given in Table 2. The solutions with negative or zero values of $R_{1}^{0}$ and $R_{2}^{0}$ do not satisfy the vacuum stability constraints:

$$
\lambda_{1}(\Phi)>0, \quad \lambda_{2}(\Phi)>0, \quad \tilde{\lambda}(\Phi)>0 .
$$

The conditions (44) must be fulfilled everywhere from the electroweak scale to the MPP scale. Otherwise another minimum of the Higgs effective potential (2) arises at some intermediate scale, destabilising the physical and MPP scale vacua.

Near the fixed points the RG equations for $R_{i}(t)$ can be linearised, i.e $R_{i}(t) \simeq R_{i}^{0}+r_{i}(t)$. The linearised system of $\mathrm{RG}$ equations for $r_{i}(t)$ can be written in the following form

$$
\frac{d r_{i}}{d t}=\left.\sum_{j=1}^{4} \frac{\partial \beta_{R_{i}}}{\partial R_{j}}\right|_{R_{i}=R_{i}^{0}} r_{j},\left.\quad \frac{\partial \beta_{R_{i}}}{\partial R_{j}}\right|_{R_{i}=R_{i}^{0}}=\frac{h_{t}^{2}}{16 \pi^{2}} a_{i j}
$$

where

$$
a_{i j}=\left(\begin{array}{cccc}
24 R_{1}^{0}-9 & 0 & 8 R_{3}^{0}+4 R_{4}^{0} & 4\left(R_{3}^{0}+R_{4}^{0}\right) \\
0 & 24 R_{2}^{0}+3 & 8 R_{3}^{0}+4 R_{4}^{0} & 4\left(R_{3}^{0}+R_{4}^{0}\right) \\
6 R_{3}^{0}+2 R_{4}^{0} & 6 R_{3}^{0}+2 R_{4}^{0} & 6\left(R_{1}^{0}+R_{2}^{0}\right)+8 R_{3}^{0}-3 & 2\left(R_{1}^{0}+R_{2}^{0}+2 R_{4}^{0}\right) \\
2 R_{4}^{0} & 2 R_{4}^{0} & 8 R_{4}^{0} & 2\left(R_{1}^{0}+R_{2}^{0}+4 R_{3}^{0}+4 R_{4}^{0}\right)-3
\end{array}\right)
$$

The fixed point is stable when all the eigenvalues of the matrix $a_{i j}$ are positive. Only in this case do all the $r_{i}(t)$ tend to zero in the infrared region.

The analysis of the convergence of the solutions of the linearised system of RG equations (45), in the vicinity of the fixed points listed in Table 2, reveals that there is only one stable fixed point solution which corresponds to

$$
R_{1}=\frac{3}{4}, \quad R_{2}=\frac{\sqrt{65}-1}{8} \simeq 0.883, \quad R_{3}=0, \quad R_{4}=0 .
$$


Choosing a large value of the top quark Yukawa coupling at the scale $\Lambda\left(\right.$ say $\left.h_{t}^{2}(\Lambda)=10\right)$ and using the fixed point solution (46) as a boundary condition for the RG equations, one establishes the position of the quasi-fixed point at the electroweak scale. If the MPP scale is close to $M_{P l}$ we get

$$
\begin{gathered}
\rho_{t}\left(M_{t}\right) \simeq 1.174, \quad \rho_{1}\left(M_{t}\right) \simeq 0.341, \quad \rho_{2}\left(M_{t}\right) \simeq 0.694 \\
\rho_{3}\left(M_{t}\right) \simeq-0.011, \quad \rho_{4}\left(M_{t}\right) \simeq-0.013 .
\end{gathered}
$$

It turns out that, for large values of the top quark Yukawa coupling at the scale $\Lambda$, the allowed range of the Higgs self-couplings is rather strongly constrained. Stringent constraints on $\lambda_{i}(\Lambda)$ come from the MPP conditions (19). Using the equations $\tilde{\lambda}(\Lambda)=0$ and $\beta_{\tilde{\lambda}}(\Lambda)=0$, one can express $\lambda_{3}(\Lambda)$ and $\lambda_{4}(\Lambda)$ in terms of the other gauge, Yukawa and Higgs self-couplings, i.e.

$$
\begin{gathered}
\lambda_{3}(\Lambda)=-\sqrt{\lambda_{1}(\Lambda) \lambda_{2}(\Lambda)}-\lambda_{4}(\Lambda), \\
\lambda_{4}^{2}(\Lambda)=\frac{6 h_{t}^{4}(\Lambda) \lambda_{1}(\Lambda)}{\left(\sqrt{\lambda_{1}(\Lambda)}+\sqrt{\lambda_{2}(\Lambda)}\right)^{2}}-2 \lambda_{1}(\Lambda) \lambda_{2}(\Lambda) \\
-\frac{3}{8}\left(3 g_{2}^{4}(\Lambda)+2 g_{2}^{2}(\Lambda) g_{1}^{2}(\Lambda)+g_{1}^{4}(\Lambda)\right),
\end{gathered}
$$

where $\lambda_{4}(\Lambda)<0$. Thus the RG flow of the Higgs self-couplings, in the MPP inspired 2HDM with an approximate generalised Peccei-Quinn symmetry, is determined by $h_{t}(\Lambda)$, $\lambda_{1}(\Lambda)$ and $\lambda_{2}(\Lambda)$. Varying $\lambda_{1}(\Lambda)$ and $\lambda_{2}(\Lambda)$, one can obtain the restrictions on their values. Because $\lambda_{4}(\Lambda)$ is a real quantity, Eq. (49) limits the allowed range of $\lambda_{1}(\Lambda)$ and $\lambda_{2}(\Lambda)$ from above. For instance, when $\lambda_{1}(\Lambda)=\lambda_{2}(\Lambda)=\lambda_{0}$ the quantity $\lambda_{4}^{2}(\Lambda)$ remains positive only if $\lambda_{0}<\frac{\sqrt{3}}{2} h_{t}^{2}(\Lambda)$. The lower bound on the Higgs self-couplings originates from the vacuum stability conditions (44). Indeed, if $\lambda_{1}(\Lambda)=\lambda_{2}(\Lambda)=\lambda_{0}$ is sufficiently small then $\tilde{\lambda}(\mu)$ tends to be negative at some intermediate scale, destabilising the physical and MPP scale vacua. Our numerical studies show that, for $\Lambda=M_{P l}$ and $R_{1}\left(M_{P l}\right)=R_{2}\left(M_{P l}\right)=R_{0}$, the value of $R_{0}$ can vary only within a very narrow interval from 0.79 to 0.87 if $h_{t}(\Lambda) \gtrsim 1.5$. Moreover the allowed range of $R_{0}$ shrinks further when $h_{t}(\Lambda)$ increases. For $h_{t}(\Lambda) \gtrsim 2.5$ the value of $R_{0}$ can vary only between 0.83 and 0.87 .

In Figs. $2 \mathrm{a}$ and $2 \mathrm{~b}$ we present the restrictions on the Higgs self-couplings $\lambda_{1}(\Lambda)$ and $\lambda_{2}(\Lambda)$ for $h_{t}(\Lambda)=3$ and two different values of the MPP scale $\Lambda=M_{P l}$ and $\Lambda=10 \mathrm{TeV}$. In these plots the allowed region of the parameter space in the $R_{1}(\Lambda)-R_{2}(\Lambda)$ plane is limited by the dotted and solid lines. The dotted line represents the vacuum stability constraints (44). For any point in the $R_{1}(\Lambda)-R_{2}(\Lambda)$ plane below the dotted curve, the vacuum stability conditions are violated at some intermediate scale between $\Lambda$ and $M_{t}$ preventing the consistent implementation of the MPP. The solid line constrains the allowed range of the Higgs self-couplings from above. For any point above this line $\lambda_{4}^{2}(\Lambda)$ 
is negative. From Fig. 2a one can see that vacuum stability and MPP conditions set stringent constraints on the Higgs self-couplings for $R_{1}(\Lambda)>0.3$, if the MPP scale is relatively high. In the considered case only a very narrow strip in the $R_{1}(\Lambda)-R_{2}(\Lambda)$ plane is not ruled out. When the MPP scale decreases, the allowed range of $\lambda_{1}(\Lambda)$ and $\lambda_{2}(\Lambda)$ enlarges. From Fig. 2b it follows that the position of the solid line does not change significantly with decreasing scale $\Lambda$, whereas the restrictions on the Higgs self-couplings caused by the vacuum stability constraints (44) become less stringent. It is worth noticing here that, independently of the MPP scale, the stable fixed point (46), which is shown as an open circle in Fig. 2, always lies in the allowed region of parameter space.

In Fig. 3 we plot the RG flow of the Higgs self-couplings from $\Lambda=M_{P l}$ to the electroweak scale. As boundary conditions we use a set of points $R_{1}(\Lambda)$ and $R_{2}(\Lambda)$ from the allowed part of the parameter space shown in Fig. 2a. The Higgs self-couplings $\lambda_{3}(\Lambda)$ and $\lambda_{4}(\Lambda)$ are chosen so that the MPP conditions (48)-(49) are fulfilled. Figs. 3a and 3b demonstrate that the trajectories, which represent different solutions of the RG equations for $\lambda_{1}(\mu), \lambda_{2}(\mu)$, and $\lambda_{3}(\mu)$, are focused in a narrow region near the quasi-fixed points at low energies. At the same time the trajectories in the $R_{4}(\mu)-R_{1}(\mu)$ plane are rather spread out in the infrared region (see Fig. 3c). This is an indication that the solutions of the RG equations for $\lambda_{4}(\mu)$ are attracted very weakly to the corresponding quasi-fixed point.

In Fig. 4 we examine the convergence of the solutions of the RG equations to the quasi-fixed points, as a function of the MPP scale. In Figs. $4 \mathrm{a}-4 \mathrm{~d}$ we set $R_{1}(\Lambda)=0.75$ and $R_{2}(\Lambda)=0.883$ and choose $R_{3}(\Lambda)$ and $R_{4}(\Lambda)$ so that the MPP conditions (48)-(49) are satisfied. The solid and dashed lines in Fig. 4 represent the dependence of the $R_{i}\left(M_{t}\right)$ on the scale $\Lambda$ for $h_{t}^{2}(\Lambda)=10$ and $h_{t}^{2}(\Lambda)=2.25$ respectively. From Fig. 4a and $4 \mathrm{~b}$ one can see that $\lambda_{1}\left(M_{t}\right)$ and $\lambda_{2}\left(M_{t}\right)$ do not change substantially, when $h_{t}^{2}(\Lambda)$ varies from 10 to 2.25. This demonstrates the good convergence rate of the solutions of the RG equations for $\lambda_{1}(\mu)$ and $\lambda_{2}(\mu)$ to the corresponding quasi-fixed points. The values of $\lambda_{3}\left(M_{t}\right)$ are quite sensitive to the choice of the MPP scale and $h_{t}(\Lambda)$ (see Fig. 4c). If $\Lambda$ is relatively high $\left(\Lambda \gtrsim 10^{13} \mathrm{GeV}\right)$, the solutions of the RG equations for $\lambda_{3}(\mu)$ are gathered near zero at the electroweak scale. But for relatively low $\Lambda\left(\Lambda \lesssim 10^{3} \mathrm{TeV}\right)$, the value of $\lambda_{3}\left(M_{t}\right)$ changes considerably when $h_{t}^{2}(\Lambda)$ is reduced from 10 to 2.25 . In general the convergence of the solutions of the RG equations for $\lambda_{1}(\mu), \lambda_{2}(\mu)$ and $\lambda_{3}(\mu)$ becomes worse when the MPP scale decreases. Finally Fig. 4d indicates a strong dependence of $\lambda_{4}\left(M_{t}\right)$ on the scale $\Lambda$ and $h_{t}^{2}(\Lambda)$, which makes it rather difficult to get any reasonable prediction for the value of this Higgs self-coupling at the electroweak scale. 


\subsection{Higgs masses and couplings}

Relying on the results of the analysis of the RG flow in the MPP inspired 2HDM, we can explore the Higgs spectrum at the electroweak scale. The Higgs sector of the 2HDM involves two charged and three neutral scalar states. Since our MPP solutions conserve $\mathrm{CP}$, one of the neutral Higgs bosons is purely $\mathrm{CP}$-odd. The charged and pseudoscalar Higgs states gain masses:

$$
m_{\chi^{ \pm}}^{2}=m_{A}^{2}-\frac{\lambda_{4}}{2} v^{2}, \quad m_{A}^{2}=\frac{2 m_{3}^{2}}{\sin 2 \beta} .
$$

In the case of the MPP solution (II), the direct searches for the rare B-meson decays $\left(B \rightarrow X_{s} \gamma\right)$ place a lower limit on the charged Higgs boson mass [76]-[77]: $m_{\chi^{ \pm}}>350 \mathrm{GeV}$.

The $\mathrm{CP}$-even states are mixed and form a $2 \times 2$ mass matrix. It is convenient to introduce a new field space basis $(h, H)$ rotated by the angle $\beta$ with respect to the initial one:

$$
\begin{aligned}
& H_{1}^{0}=\left(h \cos \beta-H \sin \beta+v_{1}\right), \\
& H_{2}^{0}=\left(h \sin \beta+H \cos \beta+v_{2}\right) .
\end{aligned}
$$

Then the field $h$ is the analogue of the SM Higgs field with vacuum expectation value $<h>=v$ and is solely responsible for the symmetry breaking, while the field $H$ has zero vacuum expectation value and is irrelevant for symmetry breaking. In this new basis the mass matrix of the Higgs scalars takes the form (see also [78]-[80])

$$
\begin{aligned}
& M^{2}=\left(\begin{array}{cc}
M_{11}^{2} & M_{12}^{2} \\
M_{21}^{2} & M_{22}^{2}
\end{array}\right)=\left(\begin{array}{ll}
\frac{\partial^{2} V}{\partial v^{2}} & \frac{1}{v} \frac{\partial^{2} V}{\partial v \partial \beta} \\
\frac{1}{v} \frac{\partial^{2} V}{\partial v \partial \beta} & \frac{1}{v^{2}} \frac{\partial^{2} V}{\partial \beta^{2}}
\end{array}\right), \\
& M_{11}^{2}=\left(\lambda_{1} \cos ^{4} \beta+\lambda_{2} \sin ^{4} \beta+\frac{\lambda}{2} \sin ^{2} 2 \beta\right) v^{2}, \\
& M_{12}^{2}=M_{21}^{2}=\frac{v^{2}}{2}\left(-\lambda_{1} \cos ^{2} \beta+\lambda_{2} \sin ^{2} \beta+\lambda \cos 2 \beta\right) \sin 2 \beta, \\
& M_{22}^{2}=m_{A}^{2}+\frac{v^{2}}{4}\left(\lambda_{1}+\lambda_{2}-2 \lambda\right) \sin ^{2} 2 \beta,
\end{aligned}
$$

where $\lambda=\lambda_{3}+\lambda_{4}$. The masses of the two $\mathrm{CP}$-even eigenstates obtained by diagonalizing the matrix (52) are given by

$$
m_{h_{1}, h_{2}}^{2}=\frac{1}{2}\left(M_{11}^{2}+M_{22}^{2} \mp \sqrt{\left(M_{22}^{2}-M_{11}^{2}\right)^{2}+4 M_{12}^{4}}\right) .
$$

The qualitative pattern of the Higgs spectrum depends very strongly on the mass $m_{A}$ of the pseudoscalar Higgs boson. With increasing $m_{A}$ the masses of all the Higgs particles grow. At very large values of $m_{A}\left(m_{A}^{2}>>v^{2}\right)$, the lightest Higgs boson mass approaches its theoretical upper limit $\sqrt{M_{11}^{2}}$. 
In the rotated field basis $(h, H)$ the trilinear part of the Lagrangian, which determines the interactions of the neutral Higgs states with the $Z$-boson, is simplified [81]-[83]:

$$
L_{A Z H}=\frac{\bar{g}}{2} M_{Z} Z_{\mu} Z_{\mu} h+\frac{\bar{g}}{2} Z_{\mu}\left[H\left(\partial_{\mu} A\right)-\left(\partial_{\mu} H\right) A\right] .
$$

where $\bar{g}=\sqrt{g_{2}^{2}+g_{1}^{2}}$. Following the traditional notations we define normalised $R$ couplings of the neutral Higgs states to vector bosons as follows: $g_{V V h_{i}}=R_{V V h_{i}} \times \mathrm{SM}$ coupling (i.e. $\left.\frac{\bar{g}}{2} M_{V}\right) ; g_{Z A h_{i}}=\frac{\bar{g}}{2} R_{Z A h_{i}}$, where $V$ is a $W^{ \pm}$or a $Z$ boson. The relative couplings $R_{Z Z h_{i}}$ and $R_{Z A h_{i}}$ are given in terms of the angles $\alpha$ and $\beta$ [84]:

$$
\begin{gathered}
R_{Z Z h_{1}}=R_{W W h_{1}}=-R_{Z A h_{2}}=\sin (\beta-\alpha), \\
R_{Z Z h_{2}}=R_{W W h_{2}}=R_{Z A h_{1}}=\cos (\beta-\alpha),
\end{gathered}
$$

where the angle $\alpha$ is defined as follows:

$$
\begin{gathered}
h_{1}=-\left(H_{1}^{0}-v_{1}\right) \sin \alpha+\left(H_{2}^{0}-v_{2}\right) \cos \alpha, \\
h_{2}=\left(H_{1}^{0}-v_{1}\right) \cos \alpha+\left(H_{2}^{0}-v_{2}\right) \sin \alpha, \\
\tan \alpha=\frac{\left(\lambda v^{2}-m_{A}^{2}\right) \sin \beta \cos \beta}{m_{A}^{2} \sin ^{2} \beta+\lambda_{1} v^{2} \cos ^{2} \beta-m_{h_{1}}^{2}} .
\end{gathered}
$$

The absolute values of the $R$-couplings $R_{V V h_{i}}$ and $R_{Z A h_{i}}$ vary from zero to unity.

The couplings of the Higgs eigenstates to the top quark $g_{t \bar{t} h_{i}}$ can also be presented as a product of the corresponding SM coupling and the $R$-coupling $R_{t \bar{t} h_{i}}$ :

$$
R_{t \bar{t} h_{1}}=\frac{\cos \alpha}{\sin \beta}, \quad R_{t \bar{t} h_{2}}=\frac{\sin \alpha}{\sin \beta} .
$$

Since the $R_{t \bar{t} h_{i}}$ are inversely proportional to $\sin \beta$ and near the quasi-fixed point $\tan \beta \lesssim 1$, the values of $R_{t \bar{t} h_{i}}$ can be substantially larger than unity.

As follows from Eqs. (50)-(57), the spectrum and couplings of Higgs bosons in the MPP inspired 2HDM, with softly broken Peccei-Quinn and $Z_{2}$ symmetry, is parametrized in terms of $m_{A}$, $\tan \beta$ and four Higgs self-couplings $\lambda_{1}\left(M_{t}\right), \lambda_{2}\left(M_{t}\right), \lambda_{3}\left(M_{t}\right)$ and $\lambda_{4}\left(M_{t}\right)$. In our study of the phenomenology of the Higgs sector, we concentrate on the quasi-fixed point scenario. In particular, at the MPP scale we set $R_{1}(\Lambda)=0.75, R_{2}(\Lambda) \simeq 0.883$ and $h_{t}^{2}(\Lambda)=10$, which correspond to the quasi-fixed point solution. At the same time we do not keep $R_{3}(\Lambda)=R_{4}(\Lambda)=0$. Instead, we find appropriate values of $\lambda_{3}(\Lambda)$ and $\lambda_{4}(\Lambda)$ that obey the MPP conditions (48)-(49). Then we evolve the top quark Yukawa and Higgs couplings down to the electroweak scale. The results of our calculations have already been discussed in the previous sections. According to our analysis near the quasi-fixed points, the Higgs self-couplings, the top quark Yukawa coupling and $\tan \beta$ depend only on the MPP scale (see Figs. 1 and 4). As a result, in the considered scenario, all Higgs masses and couplings are functions of the scale $\Lambda$ and the pseudoscalar mass $m_{A}$. Therefore, at 
the next stage, we examine the dependence of the Higgs masses and couplings on the pseudoscalar mass for each fixed value of the MPP scale.

The results of our investigations are summarised in Fig. 5-7. In Fig. 5 we plot the masses and couplings of the CP-even Higgs eigenstates for the MPP scale $\Lambda=M_{P l}$. From Fig. 5a it is clear that the masses of the Higgs particles change considerably when $m_{A}$ varies. In particular, the masses of the heaviest $\mathrm{CP}$-even and charged Higgs states rise with increasing pseudoscalar mass. At large values of $m_{A} \gtrsim 300 \mathrm{GeV}$, the corresponding Higgs states are almost degenerate around $m_{A}$. The mass of the lightest CP-even Higgs boson is not so sensitive to the variations of the pseudoscalar mass. It varies from $80 \mathrm{GeV}$ to $120 \mathrm{GeV}$. The lightest Higgs scalar $h_{1}$ can be identified as being predominantly the SM-like superposition $h$ of the neutral components of Higgs doublets, because its relative coupling to a $Z$ pair is always close to unity (see Fig. 5b). The contribution of the orthogonal combination of neutral components of Higgs doublets $H$ to $h_{1}$ is considerably smaller. As a result the coupling of the lightest CP-even Higgs state to the Higgs pseudoscalar and $Z$ is suppressed. But, at low values of $m_{A} \lesssim 100 \mathrm{GeV}$, the $\mathrm{R}$-coupling $R_{Z A h_{1}}$ is still large enough that the lightest $\mathrm{CP}-$ even and pseudoscalar Higgs states could have been produced in $e^{+} e^{-}$collisions at LEP. Because $R_{Z Z h_{1}}$ is rather close to unity, the associated production of the lightest Higgs scalar and the $Z$ boson would also have been possible. Consequently the non-observation of the SM-like Higgs particle at LEP rules out most of the parameter space near the quasi-fixed point solution if the scale $\Lambda$ is relatively high, i.e. $\Lambda \gtrsim 10^{15}$. This is a consequence of the stringent bound on the mass of the SM-like Higgs caused by the RG flow of Higgs self-couplings from $\Lambda$ to the electroweak scale. In Fig. 6 the theoretical upper bound on $m_{h_{1}}$ as a function of the MPP scale $\Lambda$ is presented. If $\Lambda \gtrsim 10^{10} \mathrm{GeV}$ the lightest $\mathrm{CP}$-even Higgs boson is lighter than $125 \mathrm{GeV}$. The upper bound on $m_{h_{1}}$ grow from $125 \mathrm{GeV}$ to $140 \mathrm{GeV}$, when the MPP scale is lowered from $10^{10} \mathrm{GeV}$ to $10^{7} \mathrm{GeV}$ (see Fig. 6)

When $\Lambda$ is near the Planck scale the $H_{1}^{0}$ component of the lightest CP-even Higgs scalar is larger than the $H_{2}^{0}$ component for $m_{A}<400 \mathrm{GeV}$. This is essentially because, at large values of the MPP scale, $\lambda_{1}\left(M_{t}\right)$ is less than $\lambda_{2}\left(M_{t}\right)$ while $v_{1} \simeq v_{2}$ in the vicinity of the quasi-fixed point. Since $H_{1}^{0}$ is the larger component, the coupling of the lightest $\mathrm{CP}_{-}$ even Higgs eigenstate $h_{1}$ to the top quark is smaller than the coupling of the heaviest one for $m_{A}<400 \mathrm{GeV}$. The dependence of the relative couplings of the $\mathrm{CP}$-even Higgs bosons to the top quark, $R_{t \bar{t} h_{i}}$, on $m_{A}$ is examined in Fig. 5c. From this figure one can see that $R_{t \bar{t} h_{2}}$ is more than twice as big as $R_{t \bar{t} h_{1}}$ at low values of $m_{A} \lesssim 100 \mathrm{GeV}$. However such small values of the pseudoscalar mass are excluded by the unsuccessful Higgs searches at LEP. With increasing $m_{A}$ the heaviest $\mathrm{CP}$-even, $\mathrm{CP}$-odd and charged Higgs states decouple. As a consequence, the couplings of the lightest Higgs boson to a $Z$ pair and to 
the top quark approach the SM ones (see Fig. 5 c), i.e. $h_{1} \simeq h$. Our numerical analysis reveals that for $\Lambda \gtrsim 10^{8} \mathrm{GeV}$ the relative coupling $R_{t \bar{t} h_{1}} \lesssim 1$.

The situation changes significantly when the MPP scale is relatively low. In Fig. 7 we study the dependence of the masses and couplings of the Higgs bosons on $m_{A}$ for the MPP scale $\Lambda=100 \mathrm{TeV}$. As before the masses of the heaviest $\mathrm{CP}$-even, $\mathrm{CP}$-odd and charged Higgs states are set by $m_{A}$. When $m_{A}$ grows, all Higgs masses increase and at large values of the pseudoscalar Higgs mass $m_{\chi^{ \pm}} \simeq m_{h_{2}} \simeq m_{A}$ (see Fig. 7a). However the upper bound on the lightest $\mathrm{CP}$-even Higgs scalar mass $m_{h_{1}}$ increases significantly. If $\Lambda \simeq 100 \mathrm{TeV}$ the upper bound on the mass of the lightest CP-even Higgs state changes from $140 \mathrm{GeV}$ to $180 \mathrm{GeV}$. Once again the main contribution to the wave function of the lightest Higgs scalar corresponds to the SM-like superposition of neutral components of Higgs doublets $h$ so that $R_{Z Z h_{1}} \approx 1$ (see Fig. $7 \mathrm{~b}$ ). However the values of $m_{A} \gtrsim M_{Z}$ are not excluded by LEP data, because the associated lightest Higgs scalar production with either a $Z$ boson or a Higgs pseudoscalar is kinematically forbidden. At low values of the pseudoscalar mass $\left(m_{A}<250 \mathrm{GeV}\right)$ the $H_{2}^{0}$ component of the lightest CP-even Higgs state is now larger than the $H_{1}^{0}$ component. Despite $\lambda_{2}\left(M_{t}\right)$ still being larger than $\lambda_{1}\left(M_{t}\right)$, the vacuum expectation value $v_{2}$ becomes considerably smaller than $v_{1}(\tan \beta \sim 0.5)$ resulting in $\lambda_{1} v_{1}^{2}>\lambda_{2} v_{2}^{2}$. This gives rise to a realignment in the Higgs spectrum. As can be seen from Fig. $7 \mathrm{c}$, the change in content of $h_{1}$ leads to a substantial increase in the coupling of the lightest Higgs scalar to the top quark. Our numerical studies demonstrate that, for values of the MPP scale $\Lambda$ below $1000 \mathrm{TeV}$, there is some range of $m_{A}$ in the quasi-fixed point scenario where $R_{t \bar{t} h_{1}} \gtrsim R_{t \bar{t} h_{2}} \gtrsim 1$. Due to the significant growth of the coupling of the lightest CP-even Higgs state to the top quark, the production cross section of the SM-like Higgs in the $2 \mathrm{HDM}$ can be $1.5-2$ times larger than in the SM [60]-662]. The enhanced production of the SM-like Higgs boson allows us to distinguish the quasi-fixed point scenario in the MPP inspired 2HDM with low MPP scale from the SM and its supersymmetric extensions, even if the extra Higgs states are heavy $\left(m_{A} \gtrsim 400-500 \mathrm{GeV}\right)$.

\section{Other MPP solutions}

The MPP solution that corresponds to the set of degenerate vacua, in which the energy density vanishes near the scale $\Lambda$ for any $\omega$, might not be a unique one in the two Higgs doublet extension of the SM. Indeed, in Appendix B we present the derivation of other MPP conditions, which correspond to the set of vacua which have zero vacuum energy density at the MPP scale for any choice of $\theta$ or $\gamma$. These scenarios were not discussed in our previous article [41], where we considered the implementation of the multiple point principle in the two Higgs doublet model of type II. 
It turns out that it is quite difficult to achieve a self-consistent realization of these other MPP solutions, if we restrict our consideration to the simplest two Higgs doublet extension of the SM with a minimal matter content. Indeed, in the Higgs field basis where only $H_{2}$ couples to the $t$-quark at the MPP scale, the observed mass hierarchy within the third generation of fermions implies that $h_{t}(\Lambda)>>g_{b}(\Lambda), g_{\tau}(\Lambda)$. At the same time, for small values of these Peccei-Quinn symmetry violating Yukawa couplings, some of the MPP conditions derived in the Appendix B cannot be satisfied.

For instance, let us consider the MPP conditions (B.8), that result in the vacuum configuration in which the energy density goes to zero for arbitrary values of the ratio of the Higgs vacuum expectation values $\Phi_{2} / \Phi_{1}$ when $\lambda_{4}(\Lambda)<\left|\lambda_{5}(\Lambda)\right|$. Substituting the explicit expressions for $\beta_{\lambda_{3}}, \beta_{\lambda_{4}}$ and $\beta_{\lambda_{5}}$ in the last MPP condition of Eqs. (B.8), we find

$$
\begin{gathered}
\beta_{\lambda_{3}}(\Lambda)+\beta_{\lambda_{4}}(\Lambda)+\operatorname{Re} \beta_{\lambda_{5}}(\Lambda)=\frac{1}{16 \pi^{2}}\left[2 \lambda_{3}^{2}(\Lambda)+4 \lambda_{5}^{2}(\Lambda)+\frac{9}{4} g_{2}^{4}(\Lambda)+\right. \\
+\frac{3}{2} g_{2}^{2}(\Lambda) g_{1}^{2}(\Lambda)+\frac{3}{4} g_{1}^{4}(\Lambda)-24\left|h_{b}(\Lambda)\right|^{2}\left|g_{b}(\Lambda)\right|^{2}-8\left|h_{\tau}(\Lambda)\right|^{2}\left|g_{\tau}(\Lambda)\right|^{2}- \\
\left.-\left(6 h_{b}^{2}(\Lambda) g_{b}^{* 2}(\Lambda)+2 h_{\tau}^{2}(\Lambda) g_{\tau}^{* 2}(\Lambda)+\text { h.c. }\right)\right]=0 .
\end{gathered}
$$

Here we have redefined the Higgs fields, so that $\lambda_{5}(\Lambda)$ is real and negative. From Eq. (58) it becomes clear that the positive contribution of the Higgs and gauge couplings to the corresponding combination of $\beta$-functions cannot be compensated by the negative contribution coming from the Yukawa interactions if $g_{b}(\Lambda) \sim g_{\tau}(\Lambda) \sim 10^{-2}$, unless $\left|h_{b}(\Lambda)\right|^{2}$ or $\left|h_{\tau}(\Lambda)\right|^{2} \gtrsim 10$. However such large values of $\left|h_{b}(\Lambda)\right|$ and $\left|h_{\tau}(\Lambda)\right|$ would spoil the validity of perturbation theory.

Due to similar reasons, it is not possible to achieve the degeneracy of vacua with respect to $\theta$. The MPP conditions that ensure the existence of such a set of degenerate minima of the Higgs effective potential at the MPP scale are given by Eqs. (B.13). After the substitution of explicit expressions for $\beta_{\lambda_{4}}$ and $\beta_{\lambda_{5}}$, one of the MPP conditions, $\beta_{\lambda_{4}}(\Lambda)+\operatorname{Re} \beta_{\lambda_{5}}(\Lambda)=0$, reduces to

$$
\begin{gathered}
12\left|h_{t}(\Lambda)\right|^{2}\left|h_{b}(\Lambda)\right|^{2}+3 g_{2}^{2}(\Lambda) g_{1}^{2}(\Lambda)-12\left|h_{b}(\Lambda)\right|^{2}\left|g_{b}(\Lambda)\right|^{2}-4\left|h_{\tau}(\Lambda)\right|^{2}\left|g_{\tau}(\Lambda)\right|^{2}- \\
-\left(6 h_{b}^{2}(\Lambda) g_{b}^{* 2}(\Lambda)+2 h_{\tau}^{2}(\Lambda) g_{\tau}^{* 2}(\Lambda)+\text { h.c. }\right)=0 .
\end{gathered}
$$

To satisfy the MPP condition (59), either $g_{b}(\Lambda)$ or $g_{\tau}(\Lambda)$ should be large. This makes the generation of the observed mass hierarchy rather problematic.

Nevertheless there is one new set of the MPP conditions whose realisation does not require large Peccei-Quinn symmetry violating Yukawa couplings. Indeed the MPP conditions (B.6), which lead to the presence of vacua in which the energy density tends to zero for any ratio of the Higgs vacuum expectation values $\Phi_{2} / \Phi_{1}$ at the MPP scale 
when $\lambda_{4}(\Lambda)>\left|\lambda_{5}(\Lambda)\right|$, can be fulfilled even if $g_{b}(\Lambda)$ and $g_{\tau}(\Lambda)$ are negligibly small. The corresponding set of degenerate minima of the Higgs effective potential

$$
<H_{1}>=\left(\begin{array}{c}
0 \\
\Phi_{1}
\end{array}\right), \quad<H_{2}>=\left(\begin{array}{c}
\Phi_{2} \\
0
\end{array}\right), \quad \Phi_{1}^{2}+\Phi_{2}^{2}=\Lambda^{2},
$$

arises if the Higgs self-couplings $\lambda_{1}(\Lambda), \lambda_{2}(\Lambda), \lambda_{3}(\Lambda)$ and their $\beta$-functions $\beta_{\lambda_{1}}(\Lambda), \beta_{\lambda_{2}}(\Lambda)$, $\beta_{\lambda_{3}}(\Lambda)$ vanish. The vanishing of the three $\beta$-functions (see Eq. (A.2)) for the Higgs selfcouplings $\lambda_{1}, \lambda_{2}$ and $\lambda_{3}$ at the MPP scale can be achieved only if the Yukawa couplings of the third generation obey two relationships:

$$
\begin{gathered}
\left|h_{t}(\Lambda)\right|^{4}=\left|h_{b}(\Lambda)\right|^{4}+\frac{1}{3}\left|h_{\tau}(\Lambda)\right|^{4}, \\
\left|h_{t}(\Lambda)\right|^{4}=\left|h_{t}(\Lambda)\right|^{2}\left|h_{b}(\Lambda)\right|^{2}+\frac{1}{4} g_{2}^{2}(\Lambda) g_{1}^{2}(\Lambda) .
\end{gathered}
$$

In Eq. (61) we neglect $g_{b}(\Lambda)$ and $g_{\tau}(\Lambda)$.

The relations (61) allows us to express the $b$-quark and $\tau$-lepton Yukawa couplings at the scale $\Lambda$ in terms of $h_{t}(\Lambda)$. Thus, for each fixed value of the top quark Yukawa coupling at the MPP scale, one can calculate the RG flow of $h_{t}(\mu), h_{b}(\mu)$ and $h_{\tau}(\mu)$ from the scale $\Lambda$ to $\mu=M_{t}$. Then Eq. (40) can be used for the determination of $\tan \beta$. Since at the electroweak scale $h_{t}\left(M_{t}\right) \sim h_{b}\left(M_{t}\right) \sim h_{\tau}\left(M_{t}\right)$, the relation (40) results in large values of $\tan \beta \sim m_{t} / m_{b}$. In the considered part of the parameter space $m_{t}\left(M_{t}\right)$ is almost independent of $\tan \beta$, i.e. $m_{t}\left(M_{t}\right) \simeq h_{t}\left(M_{t}\right) v / \sqrt{2}$.

On the other hand $m_{t}\left(M_{t}\right)$ can be determined rather precisely from experiment, using the relationship between the top quark pole and running masses (33). In the MPP scenario discussed here, the top quark Yukawa coupling at the scale $\Lambda$ can be adjusted so that the observed value of $M_{t}$ is reproduced. This permits us to evaluate all Yukawa couplings at the electroweak scale and to predict the values of $\tan \beta$ and $m_{b}\left(M_{t}\right)$ using Eqs. (36), (40). The results of our numerical studies are summarised in Table 3, where we explore the dependence of $\tan \beta$ and $m_{b}\left(M_{t}\right)$ on the scale $\Lambda$. One can see that the value of $\tan \beta$ is always rather close to 50 , while $m_{b}\left(M_{t}\right)$ changes from $3.2 \mathrm{GeV}$ to $2.6 \mathrm{GeV}$ when the MPP scale grows from $10 \mathrm{TeV}$ to $M_{P l}$.

The prediction for the running $b$-quark mass at the electroweak scale can be easily improved, if we include the Peccei-Quinn symmetry violating Yukawa couplings $g_{b}$ and $g_{\tau}$. Small values of these couplings affect neither the relations between the Yukawa couplings (61) nor the running of $h_{t}(\mu), h_{b}(\mu)$ and $h_{\tau}(\mu)$. However even very small values of the corresponding couplings $\left(\sim 10^{-2}\right)$ change the predictions for $m_{b}\left(M_{t}\right)$ and $\tan \beta$ significantly. As a result one can easily reproduce the experimental value of the running $b$-quark mass, $m_{b}\left(M_{t}\right)=2.75 \pm 0.09 \mathrm{GeV}$. But even zero values of the Peccei-Quinn symmetry violating Yukawa couplings lead to a reasonable prediction for $m_{b}\left(M_{t}\right)$ for large values of $\Lambda$. 
A stringent restriction on the MPP scale in the considered scenario comes from the non-observation of the Higgs particle at LEP. In Tables 3 and 4 we examine the upper bound on the mass of the SM-like Higgs particle as a function of the scale $\Lambda$ and the Higgs self-couplings $\lambda_{4}(\Lambda)$ and $\lambda_{5}(\Lambda)$. In order to ensure that the vacua (60) are stable at the MPP scale, $\lambda_{4}(\Lambda)$ has to be positive and the absolute value of $\lambda_{5}(\Lambda)$ should be less than $\lambda_{4}(\Lambda)$. To guarantee that the Higgs effective potential is positive definite everywhere between the MPP and electroweak scales, which makes the consistent implementation of the MPP possible, the following conditions must be fulfilled:

$$
\begin{gathered}
\lambda_{1}(\Phi)>0, \quad \lambda_{2}(\Phi)>0, \\
\widehat{\lambda}(\Phi)=\sqrt{\lambda_{1}(\Phi) \lambda_{2}(\Phi)}+\lambda_{3}(\Phi)+\min \left\{0, \lambda_{4}(\Phi)-\left|\lambda_{5}(\Phi)\right|\right\}>0 .
\end{gathered}
$$

For $\lambda_{5}=0$, the inequalities $(\underline{62})-(\underline{63})$ coincide with the vacuum stability constraints derived in our previous work [41]. It turns out that $\widehat{\lambda}(\Phi)$ is only positive for any value of $\Phi$ between $\Lambda$ and $M_{t}$ when $\left|\lambda_{5}(\Lambda)\right|<0.83 \cdot \lambda_{4}(\Lambda)$. In Fig. 8a and $8 \mathrm{~b}$ we plot the running of $\lambda_{1}(\mu), \lambda_{2}(\mu)$ and $\widehat{\lambda}(\mu)$ for $\lambda_{5}=0$ and two different values of the MPP scale: $\Lambda=M_{P l}$ and $\Lambda=10 \mathrm{TeV}$.

The upper bound $m_{h}$ on the mass of the SM-like Higgs boson, which is given by

$$
\begin{gathered}
m_{h_{1}}^{2} \lesssim m_{h}^{2}=v^{2}\left(\lambda_{1}\left(M_{t}\right) \cos ^{4} \beta+\lambda_{2}\left(M_{t}\right) \sin ^{4} \beta+\frac{\lambda\left(M_{t}\right)}{2} \sin ^{2} 2 \beta\right) \\
\lambda\left(M_{t}\right)=\lambda_{3}\left(M_{t}\right)+\lambda_{4}\left(M_{t}\right)+\lambda_{5}\left(M_{t}\right)
\end{gathered}
$$

does not vary substantially when $\lambda_{4}(\Lambda)$ and $\lambda_{5}(\Lambda)$ change (see Table 4). This weak dependence of the theoretical restriction on the SM-like Higgs mass on $\lambda_{4}(\Lambda)$ and $\lambda_{5}(\Lambda)$ is a result of the suppression of their contribution to $m_{h_{1}}^{2}$ at large values of $\tan \beta$. At the same time the upper bound on $m_{h_{1}}^{2}$ decreases significantly when the MPP scale $\Lambda$ varies from $M_{P l}$ to $10 \mathrm{TeV}$. In the case when $\Lambda \simeq 10 \mathrm{TeV}$ the SM-like Higgs mass does not exceed $75 \mathrm{GeV}$. Such small values of $m_{h_{1}}$ have been already ruled out by LEP. To satisfy LEP constraints on the mass of the Higgs boson, the MPP scale should be larger than $10^{8} \mathrm{GeV}$. In the considered MPP scenario the upper bound on the SM-like Higgs mass attains its maximum value of $140 \mathrm{GeV}$ for $\Lambda \simeq M_{P l}$.

Although the MPP scenario discussed here is not excluded from the phenomenological point of view, it seems to be rather problematic to achieve the degeneracy of vacua at the MPP scale with the accuracy $v^{2} \Lambda^{2}$ in this case. Indeed, in order to guarantee that the vacua at the MPP scale are really degenerate with respect to either $\gamma$ or $\theta$, we have to require, as in the case of degeneracy of vacua with respect to $\omega$, that the masses of all the fermions and bosons should not change when $\gamma$ or $\theta$ varies. Otherwise quantum corrections to the Higgs boson potential (21) spoil the degeneracy of the considered vacua. 
In general, when the Higgs fields acquire vacuum expectation values (6) , the $S U(2) \times U(1)$ gauge bosons gain the following masses

$$
M_{1,2}^{2}=\frac{g_{2}^{2}}{2} \Phi^{2}, \quad M_{3,4}^{2}=\left[\frac{g_{2}^{2}+g_{1}^{2}}{2} \Phi^{2} \pm \sqrt{\left(\frac{g_{2}^{2}+g_{1}^{2}}{2} \Phi^{2}\right)^{2}-4 g_{2}^{2} g_{1}^{2} \Phi_{2}^{2} \Phi_{1}^{2} \sin ^{2} \theta}\right] .
$$

In the vicinity of the MPP scale $\Phi^{2}=\Lambda^{2}, \Phi_{1}=\Lambda \cos \gamma$ and $\Phi_{2}=\Lambda \sin \gamma$. Eq. (65) reveals that the degeneracy of the MPP scale vacua with respect to $\gamma$ can be achieved only for $\sin \theta=08$. This means that the MPP solution (60) which corresponds to $\sin \theta= \pm 1$ will not get, when one loop corrections are included, a true continuum of degenerate vacua to accuracy $v^{2} \Lambda^{2}$. Thus if we interpret MPP to mean that we should choose the solution with the largest number of degenerate vacua, the solution (60) is beaten by the solutions with a Lie group symmetry such as the solution (15) mainly studied in the present article. This is connected with the fact that we do not have any custodial symmetry for solution (60), which also means that it does not exclude FCNC and CP violation in the Higgs sector automatically in contrast to the solution (15). So the solution (60) for the set of vacua parameterized by $\gamma$ is disfavoured by: 1) giving formally fewer vacua, 2) not explaining the absence of FCNC and 3) generically having $\mathrm{CP}$ violation in the Higgs sector.

Eq. (65) also illustrates the fact that the vacuum energy density of the Higgs effective potential cannot be the same for different values of $\theta$, because the masses of two gauge bosons depend rather strongly on this parameter. Since the masses of the gauge bosons change when $\theta$ varies, quantum corrections would spoil the degeneracy of the MPP scale vacua with respect to $\theta$.

Because at $\sin \theta=0$ the masses of the gauge bosons are invariant under the variations of $\gamma$ and $\omega$, one can try to find a vacuum configuration in which the energy density goes to zero for arbitrary values of $\gamma$ and $\omega$ at the MPP scale. Then the independence of the vacuum energy density on the phase $\omega$ implies that the Yukawa and Higgs self-couplings obey the MPP conditions (18)-(19). In this case the degeneracy of the vacua with respect to $\gamma$ can be achieved only when $\lambda_{1}(\Lambda)=\lambda_{2}(\Lambda)=0$. However, in our previous publication [41, we argued that either $\lambda_{1}(\Phi)$ or $\tilde{\lambda}(\Phi)$ tends to be negative just below the MPP scale if $\lambda_{1}(\Lambda)=\lambda_{2}(\Lambda)=0$. As a result, near the scale $\Lambda$, there exists another minimum of the Higgs effective potential with a huge and negative vacuum energy density. This prohibits the self-consistent implementation of the MPP for arbitrary values of both $\gamma$ and $\omega$.

\footnotetext{
${ }^{8}$ One can show that in the limit $\sin \theta \rightarrow 0$ the set of degenerate vacua with respect to $\gamma$ in one field basis is equivalent to the set of degenerate vacua with respect to $\omega=2 \gamma$ in another field basis.
} 


\section{Conclusions}

In this paper we have considered the application of the multiple point principle (MPP) to the non-supersymmetric two Higgs doublet extension of the SM. In general new couplings, which appear in this model, give rise to potentially large flavour changing neutral currents and $\mathrm{CP}$-violation effects. We have argued that MPP can be used as a mechanism for the suppression of FCNC and CP-violating interactions. Indeed, MPP postulates the existence of a large set of degenerate vacua, which are allowed by a given theory. These vacua might not have exactly the same vacuum energy density. Here we assumed that the vacua at the electroweak and at the high MPP scale $\Lambda$ are degenerate with the accuracy $v^{2} \Lambda^{2}$. Normally the presence of a large set of degenerate vacua is associated with an enlarged global symmetry of the Lagrangian of the considered model. This is also the case in the 2HDM. The most favourable solution we found implies that the quartic part of the Higgs potential and the Lagrangian for the Higgs-fermion interactions are invariant under the transformations of a set of global $U(1)$ symmetries (27), which forbid nondiagonal flavour transitions and $\mathrm{CP}$ violating couplings. One example of such a custodial symmetry is a Peccei-Quinn symmetry that contains $Z_{2}$ symmetry as a subgroup. This $Z_{2}$ discrete symmetry ensures the suppression of FCNC processes in the the 2HDM of type II. At the same time MPP allows us to avoid problems that usually arise in the framework of the two Higgs doublet models with exact global $U(1)$ or $Z_{2}$ symmetries. This is because we did not require the set of vacua at the MPP scale to be exactly degenerate. Therefore global custodial symmetries appearing in the MPP inspired 2HDM can be approximate. As a consequence, in our favourable MPP solution, the breakdown of electroweak symmetry does not give rise to either an axion or domain walls. Meanwhile the custodial symmetry violating couplings are expected to be small $O\left(v^{2} / \Lambda^{2}\right)$. This leads to the suppression of FCNC and CP-violating effects.

We explored the RG flow of the Yukawa and Higgs couplings within the MPP inspired 2HDM with approximate custodial symmetries and studied the phenomenology of the Higgs sector in the framework of these models. In our analysis we concentrated on the quasi-fixed point scenarios. The positions of the quasi-fixed points at moderate and large values of $\tan \beta$ have been established. We argued that the quasi-fixed point scenarios which correspond to large $\tan \beta$ lead to unacceptably large values of the top quark running mass. Nevertheless we found a self-consistent solution when only $h_{t}(\Lambda) \gtrsim 1$, while all other Yukawa couplings are small. In this case $\tan \beta$ can be chosen so that the appropriate value of the top quark mass is reproduced. We also demonstrated that the RG solutions for the Higgs self-couplings $\lambda_{1}(\mu), \lambda_{2}(\mu)$ and $\lambda_{3}(\mu)$ are focused in a narrow interval near the quasi-fixed points at low energies, if the MPP scale is relatively high $\left(\Lambda \gtrsim 10^{13} \mathrm{GeV}\right)$. 
The solutions of the RG equations for $\lambda_{4}(\mu)$ are attracted to the quasi-fixed point rather weakly.

In the considered quasi-fixed point scenario, the spectrum and couplings of the Higgs bosons depend on the MPP scale and pseudoscalar Higgs mass $m_{A}$ predominantly. The masses of all the Higgs states rise with increasing pseudoscalar mass. At large values of $m_{A} \gtrsim 300 \mathrm{GeV}$ the heaviest $\mathrm{CP}$-even, $\mathrm{CP}$-odd and charged Higgs bosons are almost degenerate around $m_{A}$. When $m_{A}$ is large the lightest Higgs boson mass approaches its theoretical upper bound. The results of our numerical studies show that $m_{h_{1}}$ does not exceed $125 \mathrm{GeV}$ if $\Lambda \gtrsim 10^{10} \mathrm{GeV}$. At the same time, when $\Lambda \simeq 100-10 \mathrm{TeV}$, the lightest Higgs boson mass can reach $180-220 \mathrm{GeV}$. With increasing $m_{A}$ the heaviest $\mathrm{CP}-$ even, $\mathrm{CP}-$ odd and charged Higgs states decouple and the couplings of the lightest Higgs boson approach the SM ones. However our numerical analysis revealed that, for MPP scales $\Lambda$ below $1000 \mathrm{TeV}$, there is a range of $m_{A}$ where the couplings of the lightest Higgs scalar to the top quark is considerably larger than in the SM. This leads to the enhanced production of the lightest Higgs particle at the LHC, which would allow us to distinguish the quasi-fixed point scenario in the MPP inspired 2HDM from the SM and its supersymmetric extensions.

We also discussed other possible scenarios, which appear in the 2HDM as a result of the implementation of the MPP. In contrast to our favourable MPP solution, the other scenarios do not result in either exact or approximate global symmetries. Moreover, in most cases which we considered, it is extremely difficult to reproduce the observed mass hierarchy in the quark and lepton sector. Nevertheless we found one new scenario in which the corresponding MPP conditions can be fulfilled in the leading approximation. This new MPP solution leads to a set of vacua in which the energy density tends to zero for arbitrary values of the ratio of the Higgs vacuum expectation values, $\tan \gamma=\Phi_{2} / \Phi_{1}$, at the MPP scale $\left(\Lambda^{2}=\Phi_{1}^{2}+\Phi_{2}^{2}\right)$. In this scenario the SM-like Higgs boson attains its maximum value of $140 \mathrm{GeV}$ when $\Lambda \simeq M_{P l}$ whereas low values of the MPP scale $\left(\Lambda<10^{8} \mathrm{GeV}\right)$ lead to a too light Higgs boson, which has already been ruled out by LEP. However, because the considered MPP scenario is not related with the invariance of the Lagrangian under global symmetry transformations, the inclusion of the complete set of one-loop corrections to the Higgs boson potential spoils the degeneracy of vacua at the MPP scale. Therefore the degeneracy of vacua with the accuracy $v^{2} \Lambda^{2}$ cannot be achieved.

\section{Acknowledgements}

The authors are grateful to L. V. Laperashvili and M. Sher for valuable comments and remarks. We would also like to thank S. F. King, D. J. Miller, S. Moretti, L. B. Okun, M. Shifman, D. Sutherland and R. St.Denis for fruitful discussions. The authors acknowl- 
edge support from the SHEFC grant HR03020 SUPA 36878. 


\section{Appendix A: Renormalization of the Higgs self- couplings in general $2 \mathrm{HDM}$}

The structure of the renormalization group equations for the Higgs self-couplings in the $2 \mathrm{HDM}$ is fixed by the set of the $\beta$-functions

$$
\frac{d \lambda_{i}}{d t}=\beta_{\lambda_{i}}
$$

In Eq.(A.1) index $i$ runs from 1 to 7 . The variable $t$ is defined in the conventional way: $t=\ln \mu$, where $\mu$ is the renormalization scale. When $\lambda_{6}=\lambda_{7}=0$, we obtain:

$$
\begin{aligned}
& \beta_{\lambda_{1}}=\frac{1}{16 \pi^{2}}\left[12 \lambda_{1}^{2}+4 \lambda_{3}^{2}+4 \lambda_{3} \lambda_{4}+2 \lambda_{4}^{2}+2\left|\lambda_{5}\right|^{2}+\frac{9}{4} g_{2}^{4}+\frac{3}{2} g_{2}^{2} g_{1}^{2}+\frac{3}{4} g_{1}^{4}-\right. \\
& \left.-\lambda_{1}\left(3\left(3 g_{2}^{2}+g_{1}^{2}\right)-12\left|h_{b}\right|^{2}-4\left|h_{\tau}\right|^{2}\right)-12\left|h_{b}\right|^{4}-4\left|h_{\tau}\right|^{4}\right] \text {, } \\
& \beta_{\lambda_{2}}=\frac{1}{16 \pi^{2}}\left[12 \lambda_{2}^{2}+4 \lambda_{3}^{2}+4 \lambda_{3} \lambda_{4}+2 \lambda_{4}^{2}+2\left|\lambda_{5}\right|^{2}+\frac{9}{4} g_{2}^{4}+\frac{3}{2} g_{2}^{2} g_{1}^{2}+\frac{3}{4} g_{1}^{4}-\right. \\
& \left.-\lambda_{2}\left(3\left(3 g_{2}^{2}+g_{1}^{2}\right)-12\left|h_{t}\right|^{2}-12\left|g_{b}\right|^{2}-4\left|g_{\tau}\right|^{2}\right)-12\left|h_{t}\right|^{4}-12\left|g_{b}\right|^{4}-4\left|g_{\tau}\right|^{4}\right], \\
& \beta_{\lambda_{3}}=\frac{1}{16 \pi^{2}}\left[2\left(\lambda_{1}+\lambda_{2}\right)\left(3 \lambda_{3}+\lambda_{4}\right)+4 \lambda_{3}^{2}+2 \lambda_{4}^{2}+2\left|\lambda_{5}\right|^{2}+\frac{9}{4} g_{2}^{4}-\frac{3}{2} g_{2}^{2} g_{1}^{2}+\frac{3}{4} g_{1}^{4}-\right. \\
& -\lambda_{3}\left(3\left(3 g_{2}^{2}+g_{1}^{2}\right)-6\left|h_{t}\right|^{2}-6\left|g_{b}\right|^{2}-2\left|g_{\tau}\right|^{2}-6\left|h_{b}\right|^{2}-2\left|h_{\tau}\right|^{2}\right)-12\left|h_{t}\right|^{2}\left|h_{b}\right|^{2}- \\
& \left.-12\left|h_{b}\right|^{2}\left|g_{b}\right|^{2}-4\left|h_{\tau}\right|^{2}\left|g_{\tau}\right|^{2}\right] \\
& \beta_{\lambda_{4}}=\frac{1}{16 \pi^{2}}\left[2 \lambda_{4}\left(\lambda_{1}+\lambda_{2}+4 \lambda_{3}+2 \lambda_{4}\right)+8\left|\lambda_{5}\right|^{2}+3 g_{2}^{2} g_{1}^{2}-\lambda_{4}\left(3\left(3 g_{2}^{2}+g_{1}^{2}\right)-\right.\right. \\
& \left.-6\left|h_{t}\right|^{2}-6\left|g_{b}\right|^{2}-2\left|g_{\tau}\right|^{2}-6\left|h_{b}\right|^{2}-2\left|h_{\tau}\right|^{2}\right)+12\left|h_{t}\right|^{2}\left|h_{b}\right|^{2}-12\left|h_{b}\right|^{2}\left|g_{b}\right|^{2}- \\
& \left.-4\left|h_{\tau}\right|^{2}\left|g_{\tau}\right|^{2}\right] \\
& \beta_{\lambda_{5}}=\frac{1}{16 \pi^{2}}\left[2 \lambda_{5}\left(\lambda_{1}+\lambda_{2}+4 \lambda_{3}+6 \lambda_{4}\right)-\lambda_{5}\left(3\left(3 g_{2}^{2}+g_{1}^{2}\right)-6\left|h_{t}\right|^{2}-6\left|g_{b}\right|^{2}-\right.\right. \\
& \left.\left.-2\left|g_{\tau}\right|^{2}-6\left|h_{b}\right|^{2}-2\left|h_{\tau}\right|^{2}\right)-12 h_{b}^{2} g_{b}^{* 2}-4 h_{\tau}^{2} g_{\tau}^{* 2}\right], \\
& \beta_{\lambda_{6}}=\frac{1}{16 \pi^{2}}\left[\left(\lambda_{1}+\lambda_{3}+\lambda_{4}\right)\left(3 g_{b}^{*} h_{b}+h_{\tau} g_{\tau}^{*}\right)+\lambda_{5}\left(3 h_{b}^{*} g_{b}+h_{\tau}^{*} g_{\tau}\right)-12\left|h_{b}\right|^{2} h_{b} g_{b}^{*}-\right. \\
& \left.-4\left|h_{\tau}\right|^{2} h_{\tau} g_{\tau}^{*}\right] \\
& \beta_{\lambda_{7}}=\frac{1}{16 \pi^{2}}\left[\left(\lambda_{2}+\lambda_{3}+\lambda_{4}\right)\left(3 g_{b}^{*} h_{b}+h_{\tau} g_{\tau}^{*}\right)+\lambda_{5}\left(3 h_{b}^{*} g_{b}+h_{\tau}^{*} g_{\tau}\right)-12\left|g_{b}\right|^{2} h_{b} g_{b}^{*}-\right. \\
& \left.-4\left|g_{\tau}\right|^{2} h_{\tau} g_{\tau}^{*}\right] \text {. }
\end{aligned}
$$

The Yukawa couplings $h_{t}, g_{b}, g_{\tau}$ and $h_{b}, h_{\tau}$ appearing on the right-hand side of Eqs.(A.2) determine the strength of the interactions of the Higgs doublets $H_{2}$ and $H_{1}$ with fermions (see (4)). Eqs. (A.2) are derived by assuming that only one Higgs doublet $H_{2}$ couples to 
$t_{R}$. This can be easily achieved by the appropriate redefinition of the Higgs doublets at the MPP scale. Although $g_{t}(\Lambda)=0$, a non-zero value of this coupling can be generated below the MPP scale, due to the renormalization group flow (see (5)), in the absence of a custodial symmetry.

\section{Appendix B: the degeneracy of vacua with respect to $\tan \gamma$ or $\theta$.}

In this section we consider possible sets of degenerate minima of the Higgs effective potential with vanishing vacuum energy density, which are not related with the presence of a Peccei Quinn symmetry. In the case when there is a vacuum configuration in which the energy density tends to zero for arbitrary values of the ratio of the Higgs vacuum expectation values $\tan \gamma=\Phi_{2} / \Phi_{1}$, the terms involving different powers of $\Phi_{2}$ and $\Phi_{1}$ in the Higgs effective potential must go to zero irrespective of each other. This leads to the conditions:

$$
\left\{\begin{array}{l}
\lambda_{1}(\Lambda)=\lambda_{2}(\Lambda)=0 \\
\lambda_{3}(\Lambda)+\lambda_{4}(\Lambda) \cos ^{2} \theta+\left(\frac{\lambda_{5}(\Lambda)}{2} e^{2 i \omega} \cos ^{2} \theta+\text { h.c. }\right)=0 \\
\lambda_{k}(\Lambda) e^{i \omega} \cos \theta+\text { h.c. }=0
\end{array}\right.
$$

where $k=6,7$. The same should happen in the conditions for the extrema of the Higgs effective potential, if it attains its minimum at the considered vacuum expectation values of the Higgs fields. Applying this requirement to the conditions $\frac{\partial V}{\partial \omega}=\frac{\partial V}{\partial \theta}=0$, one obtains:

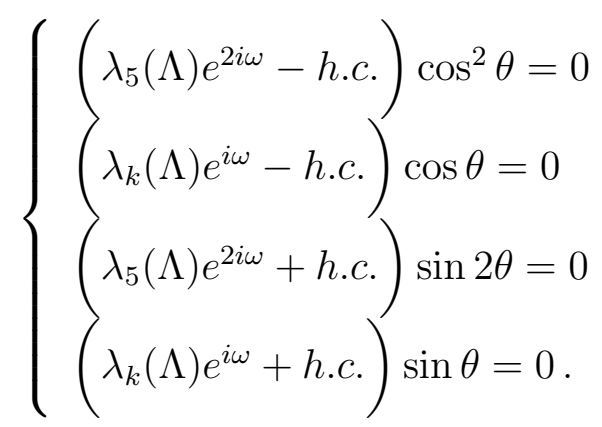

In a similar way the minimization conditions $\frac{\partial V}{\partial \Phi_{1}}=\frac{\partial V}{\partial \Phi_{2}}=0$ constrain the $\beta$-functions of the Higgs self-couplings:

$$
\left\{\begin{array}{l}
\beta_{\lambda_{1}}(\Lambda)=\beta_{\lambda_{2}}(\Lambda)=0 \\
\beta_{\lambda_{3}}(\Lambda)+\beta_{\lambda_{4}}(\Lambda) \cos ^{2} \theta+\left(\frac{\beta_{\lambda_{5}}(\Lambda)}{2} e^{2 i \omega}+\text { h.c. }\right) \cos ^{2} \theta=0 \\
\left(\beta_{\lambda_{k}}(\Lambda) e^{i \omega}+\text { h.c. }\right) \cos \theta=0 .
\end{array}\right.
$$

The relationships (B.3) for $\beta_{\lambda_{i}}$ are deduced by assuming that the conditions (B.1) are fulfilled. 
Some of the MPP conditions $(\overline{B .1})-(\overline{B .3})$ are satisfied when $\cos \theta$ goes to zero. For $\cos \theta=0$, the MPP conditions (B.1) reduce to $\lambda_{1}(\Lambda)=\lambda_{2}(\Lambda)=\lambda_{3}(\Lambda)=0$. In this limit, the Higgs effective potential near the scale $\Lambda$ can be written in the following form:

$$
\begin{gathered}
V\left(H_{1}, H_{2}\right)=\left(\lambda_{4}(\Lambda)+\left(\frac{\lambda_{5}(\Lambda)}{2} e^{2 i \omega}+\text { h.c. }\right)\right) \Phi_{1}^{2} \Phi_{2}^{2} \cos ^{2} \theta+ \\
+2\left(X^{2}+Y^{2}\right)^{1 / 2} \Phi_{2} \Phi_{1} \cos \theta \cos (\omega+\varphi),
\end{gathered}
$$

where

$$
\begin{gathered}
\varphi=\tan ^{-1}\left(\frac{Y}{X}\right), \quad X=\operatorname{Re}\left(\lambda_{6}(\Lambda) \Phi_{1}^{2}+\lambda_{7}(\Lambda) \Phi_{2}^{2}\right) \\
Y=\operatorname{Im}\left(\lambda_{6}(\Lambda) \Phi_{1}^{2}+\lambda_{7}(\Lambda) \Phi_{2}^{2}\right) .
\end{gathered}
$$

The value of $\cos \theta=0$ corresponds to the minimum of the scalar potential (B.4) only if $\lambda_{4}(\Lambda)>\left|\lambda_{5}(\Lambda)\right|>0$ and $X=Y=0$. Otherwise, $\cos \theta$ tends to get a non-zero value and the vacuum energy becomes negative. The real and imaginary parts of $\lambda_{6}(\Lambda) \Phi_{1}^{2}+\lambda_{7}(\Lambda) \Phi_{2}^{2}$ only get zero values independently of $\tan \gamma$ in the case when both $\lambda_{6}(\Lambda)$ and $\lambda_{7}(\Lambda)$ vanish identically. For $\lambda_{6}(\Lambda)=\lambda_{7}(\Lambda)=0$, the Higgs scalar potential at the MPP scale simplifies further, so that finally we get:

$$
V\left(H_{1}, H_{2}\right)=\left(\lambda_{4}(\Lambda)+\left(\frac{\lambda_{5}(\Lambda)}{2} e^{2 i \omega}+\text { h.c. }\right)\right) \Phi_{1}^{2} \Phi_{2}^{2} \cos ^{2} \theta
$$

The scalar potential (B.5) reaches a minimum at $\cos \theta=0$, where $V\left(H_{1}, H_{2}\right)$ vanishes. Substituting $\cos \theta=0$ into Eq.(B.3) , we find the MPP conditions that provide a degeneracy of vacua with different values of $\tan \gamma$ :

$$
\left\{\begin{array}{l}
\lambda_{4}(\Lambda)>\left|\lambda_{5}(\Lambda)\right| \\
\lambda_{1}(\Lambda)=\lambda_{2}(\Lambda)=\lambda_{3}(\Lambda)=\lambda_{6}(\Lambda)=\lambda_{7}(\Lambda)=0 \\
\beta_{\lambda_{1}}(\Lambda)=\beta_{\lambda_{2}}(\Lambda)=\beta_{\lambda_{3}}(\Lambda)=0
\end{array}\right.
$$

If $\cos \theta \neq 0$, then the degeneracy of vacua with respect to $\tan \gamma$ can be achieved only when $\lambda_{6}(\Lambda)=\lambda_{7}(\Lambda)=0$ (see Eq.(B.1) $-(\overline{B .2})$ ). In this case the Higgs effective potential at the MPP scale takes the form:

$$
V\left(H_{1}, H_{2}\right)=\left[\lambda_{3}(\Lambda)+\lambda_{4}(\Lambda) \cos ^{2} \theta+\left(\frac{\lambda_{5}(\Lambda)}{2} e^{2 i \omega}+\text { h.c. }\right) \cos ^{2} \theta\right] \Phi_{1}^{2} \Phi_{2}^{2},
$$

where $\lambda_{3}(\Lambda), \lambda_{4}(\Lambda)$ and $\lambda_{5}(\Lambda)$ obey Eq.(B.1) $-(\overline{B .2})$. Minima of the scalar potential (B.7) with a non-zero value of $\cos \theta$ arise when $\lambda_{4}<\left|\lambda_{5}\right|$. In this part of parameter space, the vacuum energy density decreases with increasing $\cos ^{2} \theta$ and reaches its minimum value at $\cos \theta= \pm 1$. If we redefine the Higgs fields so that $\lambda_{5}(\Lambda)$ becomes real and negative, 
the minimum of the Higgs effective potential (B.7) corresponds to $\omega=0$. Then the MPP conditions (B.1) $-(\overline{B .3})$ reduce to:

$$
\left\{\begin{array}{l}
\lambda_{4}(\Lambda)<\left|\lambda_{5}(\Lambda)\right| \\
\lambda_{1}(\Lambda)=\lambda_{2}(\Lambda)=\lambda_{6}(\Lambda)=\lambda_{7}(\Lambda)=0 \\
\lambda_{3}(\Lambda)+\lambda_{4}(\Lambda)+\lambda_{5}(\Lambda)=0 \\
\beta_{\lambda_{1}}(\Lambda)=\beta_{\lambda_{2}}(\Lambda)=\operatorname{Re} \beta_{\lambda_{6}}(\Lambda)=\operatorname{Re} \beta_{\lambda_{7}}(\Lambda)=0 \\
\beta_{\lambda_{3}}(\Lambda)+\beta_{\lambda_{4}}(\Lambda)+\operatorname{Re} \beta_{\lambda_{5}}(\Lambda)=0 .
\end{array}\right.
$$

Let us now consider vacuum configurations in which the energy density vanishes for arbitrary values of $\theta$. The Higgs effective potential will not depend on $\cos \theta$ near the MPP scale, only if the following conditions are satisfied:

$$
\begin{aligned}
& \lambda_{4}(\Lambda)+\left(\frac{\lambda_{5}(\Lambda)}{2} e^{2 i \omega}+\text { h.c. }\right)=0 \\
& \left(\lambda_{6}(\Lambda) \Phi_{1}^{2}+\lambda_{7}(\Lambda) \Phi_{2}^{2}\right) e^{i \omega}+\text { h.c. }=0 .
\end{aligned}
$$

If the relationships (B.9) between the Higgs self-couplings are fulfilled, the scalar potential can be written as:

$$
V\left(H_{1}, H_{2}\right) \simeq \frac{1}{2}\left(\sqrt{\lambda_{1}(\Lambda)} \Phi_{1}^{2}-\sqrt{\lambda_{2}(\Lambda)} \Phi_{2}^{2}\right)^{2}+\left(\sqrt{\lambda_{1}(\Lambda) \lambda_{2}(\Lambda)}+\lambda_{3}(\Lambda)\right) \Phi_{1}^{2} \Phi_{2}^{2} .
$$

Its minimum value goes to zero when

$$
\bar{\lambda}(\Lambda)=\sqrt{\lambda_{1}(\Lambda) \lambda_{2}(\Lambda)}+\lambda_{3}(\Lambda)=0
$$

If the Higgs effective potential has a local minimum at the MPP scale then $\left.\frac{d \bar{\lambda}}{d \Phi}\right|_{\Phi=\Lambda}$ vanishes as well.

The presence of the set of degenerate vacua with respect to $\theta$ implies that the terms which are proportional to $\cos \theta$ and $\cos ^{2} \theta$ in the conditions for the extrema $\frac{\partial V}{\partial \omega}=\frac{\partial V}{\partial \Phi_{1}}=\frac{\partial V}{\partial \Phi_{2}}=0$ should vanish separately. It imposes extra constraints on the Yukawa and Higgs self-couplings, which are given by:

$$
\left\{\begin{array}{l}
\frac{\lambda_{5}(\Lambda)}{2} e^{2 i \omega}-\text { h.c. }=0 \\
\left(\lambda_{6}(\Lambda) \Phi_{1}^{2}+\lambda_{7}(\Lambda) \Phi_{2}^{2}\right) e^{i \omega}-\text { h.c. }=0 \\
\beta_{\lambda_{4}}(\Lambda)+\left[\frac{\beta_{\lambda_{5}}(\Lambda)}{2} e^{2 i \omega}+\text { h.c. }\right]=0 \\
{\left[\left(3 \lambda_{6}(\Lambda) \Phi_{1}^{2}+\lambda_{7}(\Lambda) \Phi_{2}^{2}\right) \Phi_{2}+\left(\beta_{\lambda_{6}}(\Lambda) \Phi_{1}^{2}+\beta_{\lambda_{7}}(\Lambda) \Phi_{2}^{2}\right) \frac{\Phi_{1}^{2} \Phi_{2}}{\Phi^{2}}\right] e^{i \omega}+\text { h.c. }=0} \\
{\left[\left(\lambda_{6}(\Lambda) \Phi_{1}^{2}+3 \lambda_{7}(\Lambda) \Phi_{2}^{2}\right) \Phi_{1}+\left(\beta_{\lambda_{6}}(\Lambda) \Phi_{1}^{2}+\beta_{\lambda_{7}}(\Lambda) \Phi_{2}^{2}\right) \frac{\Phi_{1} \Phi_{2}^{2}}{\Phi^{2}}\right] e^{i \omega}+\text { h.c. }=0}
\end{array}\right.
$$


The first two relationships between the $\lambda_{i}(\Lambda)$ in Eq.(B.12) come from $\frac{\partial V}{\partial \omega}=0$, whereas the other three conditions follow from the assumption that $\frac{\partial V}{\partial \Phi_{1}}$ and $\frac{\partial V}{\partial \Phi_{2}}$ are independent of $\cos \theta$ near the minima of the Higgs scalar potential at the MPP scale. The derivative of $V\left(H_{1}, H_{2}\right)$ with respect to $\theta$ vanishes automatically, if the MPP conditions (B.9) are satisfied.

To simplify the analysis, we restrict our consideration to real and negative values of $\lambda_{5}(\Lambda)$, which can be arranged by the appropriate redefinition of the Higgs fields. Then the first MPP condition in Eq.(B.12) enforces $\omega$ to take a discrete set of values $\frac{\pi}{2} n$, where $n$ is an integer number. However only even values of $n$ correspond to a minimum of the Higgs scalar potential. Substituting $\omega=\pi m$ into Eq.(B.9) and Eq.(B.11)-(B.12)

$$
\left\{\begin{array}{l}
\bar{\lambda}(\Lambda)=\beta_{\bar{\lambda}}(\Lambda)=0 \\
\lambda_{4}(\Lambda)+\lambda_{5}(\Lambda)=0 \\
\operatorname{Re} \lambda_{6}(\Lambda)=\operatorname{Re} \lambda_{7}(\Lambda)=0 \\
\operatorname{Im}\left[\lambda_{6}(\Lambda) \Phi_{1}^{2}+\lambda_{7}(\Lambda) \Phi_{2}^{2}\right]=0 \\
\beta_{\lambda_{4}}(\Lambda)+\operatorname{Re} \beta_{\lambda_{5}}(\Lambda)=0 \\
\operatorname{Re}\left[\beta_{\lambda_{6}}(\Lambda) \Phi_{1}^{2}+\beta_{\lambda_{7}}(\Lambda) \Phi_{2}^{2}\right]=0
\end{array}\right.
$$

Relying on the MPP conditions (B.13) and taking into account that $V\left(H_{1}, H_{2}\right)$ vanishes near the MPP scale when $\Phi_{2}^{2}=\sqrt{\lambda_{1}(\Lambda) / \lambda_{2}(\Lambda)} \Phi_{1}^{2}$, one can easily deduce the complete expression for the vacuum energy density at the scale $\Lambda$ :

$$
\begin{aligned}
V\left(H_{1}, H_{2}\right) & \simeq \frac{1}{2}\left(\sqrt{\lambda_{1}(\Lambda)} \Phi_{1}^{2}-\sqrt{\lambda_{2}(\Lambda)} \Phi_{2}^{2}+\varkappa \Phi_{1} \Phi_{2} \cos \theta \sin \omega\right)^{2}+ \\
& +2\left(\lambda_{4}(\Lambda)-\frac{\varkappa^{2}}{4}\right) \Phi_{1}^{2} \Phi_{2}^{2} \cos ^{2} \theta \sin ^{2} \omega
\end{aligned}
$$

where

$$
\varkappa=\frac{-2 \operatorname{Im} \lambda_{6}(\Lambda)}{\sqrt{\lambda_{1}(\Lambda)}}=\frac{2 \operatorname{Im} \lambda_{7}(\Lambda)}{\sqrt{\lambda_{2}(\Lambda)}},
$$

which is valid for arbitrary values of $\gamma, \theta$ and $\omega$. From Eq.(B.14) it is obvious that the stable minima at $\omega=\pi m$, which lead to degeneracy of the vacuua with respect to $\theta$, are attained only for positive values of $\lambda_{4}(\Lambda)$ when $\lambda_{4}(\Lambda)>\varkappa^{2} / 4$. 


\section{References}

[1] N. Cabibbo, Phys. Rev. Lett. 10 (1963) 531.

[2] M. Kobayashi and T. Maskawa, Prog. Theor. Phys. 49 (1973) 652.

[3] S. L. Glashow, J. Iliopoulos and L. Maiani, Phys. Rev. D 2 (1970) 1285.

[4] Y. Grossman, Int. J. Mod. Phys. A 19 (2004) 907.

[5] D. L. Bennett, H. B. Nielsen, Int.J.Mod.Phys. A 9, 5155 (1994).

[6] D. L. Bennett, H. B. Nielsen, Int.J.Mod.Phys. A 14, 3313 (1999).

[7] D. L. Bennett, C. D. Froggatt, H. B. Nielsen, in Proceedings of the 27th International Conference on High Energy Physics, Glasgow, Scotland, 1994, p.557.

[8] J. F. Gunion, H. E. Haber, G. Kane, S. Dawson, The Higgs Hunter's Guide, (Addison-Wesley, Redwood City, CA, 1990).

[9] M. Sher, Phys. Rept. 179 (1989) 273.

[10] S. Weinberg, Phys. Rev. Lett. 63 (1989) 2333.

[11] S. Weinberg, Phys. Rev. D 42 (1990) 860.

[12] S. M. Barr and A. Zee, Phys. Rev. Lett. 65 (1990) 21.

[13] D. Chang, W. Y. Keung and T. C. Yuan, Phys. Rev. D 43 (1991) 14.

[14] J. F. Gunion and F. Vega, Phys. Lett. B 251 (1991) 211.

[15] R. C. Leigh, S. Pabin and R. M. Xu, Nucl. B Phys. 352 (1991) 45.

[16] Y. B. Zeldovich, I. Y. Kobzarev and L. B. Okun, Zh. Eksp. Teor. Fiz. 67 (1974) 3 [Sov. Phys. JETP 40 (1974) 1].

[17] A. Vilenkin, Phys. Rept. 121 (1985) 263.

[18] C. D. Froggatt and H. B. Nielsen, Phys. Lett. B 368 (1996) 96.

[19] N. Cabibbo, L. Maiani, G. Parisi, R. Petronzio, Nucl. B Phys. 158 (1979) 295.

[20] M. A. Beg, C. Panagiotakopolus, A. Sirlin, Phys. Rev. Lett. 52 (1984) 883.

[21] M. Lindner, Z. Phys. C 31 (1986) 295.

[22] P. Q. Hung, G. Isidori, Phys. Lett. B 402 (1997) 122. 
[23] T. Hambye, K. Reisselmann, Phys. Rev. D 55 (1997) 7255.

[24] M. Lindner, M. Sher, H. W. Zaglauer, Phys. Lett. B 228 (1989) 139.

[25] N. V. Krasnikov, S. Pokorski, Phys. Lett. B 288 (1991) 184.

[26] M. Sher, Phys. Lett. B 317 (1993) 159.

[27] M. Sher, Phys. Lett. B 331 (1994) 448.

[28] N. Ford, D. R. T. Jones, P. W. Stephenson, M. B. Einhorn, Nucl. Phys. B 395 (1993) 17.

[29] G. Altarelli, G. Isidori, Phys. Lett. B 337 (1994) 14.

[30] J. A. Casas, J. R. Espinosa, M. Quiros, Phys. Lett. B 342 (1995) 171.

[31] M. A. Diaz, T. A. Ter Veldius, T. J. Weiler, Phys. Rev. D 54 (1996) 5855.

[32] C. D. Froggatt and H. B. Nielsen, Surveys High Energ. Phys. 18 (2003) 55.

[33] C. D. Froggatt, H. B. Nielsen and L. V. Laperashvili, Int. J. Mod. Phys. A 20 (2005) 1268.

[34] C. D. Froggatt, PASCOS 2004, Part I: Particles, Strings and Cosmology, Boston, 2004, p.325 (World Scientific Publishing Company, 2005) arXiv:hep-ph/0412337.

[35] E. Brubaker et al. [Tevatron Electroweak Working Group], hep-ex/0608032.

[36] C. D. Froggatt, H. B. Nielsen and Y. Takanishi, Phys. Rev. D 64 (2001) 113014.

[37] R. Barate et al. [LEP Working Group for Higgs boson searches], Phys. Lett. B 565 (2003) 61.

[38] C. Froggatt, L. Laperashvili, R. Nevzorov and H. B. Nielsen, Phys. Atom. Nucl. 67 (2004) 582 [Yad. Fiz. 67 (2004) 601].

[39] C. Froggatt, L. Laperashvili, R. Nevzorov and H. B. Nielsen, arXiv:hep-ph/0411273.

[40] C. Froggatt, R. Nevzorov and H. B. Nielsen, Nucl. Phys. B 743 (2006) 133.

[41] C. D. Froggatt, L. Laperashvili, R. Nevzorov, H. B. Nielsen and M. Sher, Phys. Rev. D 73 (2006) 095005.

[42] S. L. Glashow and S. Weinberg, Phys. Rev. D 15 (1977) 1958.

[43] C. T. Hill, C. N. Leung and S. Rao, Nucl. Phys. B 262 (1985) 517; 
[44] K. Inoue, A. Kakuto, Y. Nakano, Prog. Theor. Phys. 63 (1980) 234.

[45] H. E. Haber, R. Hempfling, Phys. Rev. D 48 (1993) 4280.

[46] H. Komatsu, Prog. Theor. Phys. 67 (1982) 1177.

[47] D. Kominis, R. S. Chivukula, Phys. Lett. B 304 (1993) 152.

[48] R. Flores, M. Sher, Ann. Phys. 148 (1983) 295.

[49] A. Bovier, D. Wyler, Phys. Lett. B 154 (1985) 43.

[50] A. J. Davies, G. C. Joshi, Phys. Rev. Lett. 58 (1987) 1919.

[51] J. Maalampi, J. Sirkka, I. Vilja, Phys. Lett. B 265 (1991) 371.

[52] S. Kanemura, T. Kubota, E. Takasugi, Phys. Lett. B 313 (1993) 155.

[53] A. G. Akeroyd, A. Arhrib, E. Naimi, Phys. Lett. B 490 (2000) 119.

[54] S. Nie and M. Sher, Phys. Lett. B 449 (1999) 89.

[55] S. Kanemura, T. Kasai and Y. Okada, Phys. Lett. B 471 (1999) 182.

[56] T. P. Cheng and M. Sher, Phys. Rev. D 35 (1987) 3484.

[57] J. Bagger, S. Dimopoulos and E. Masso, Phys. Lett. B 156 (1985) 357.

[58] C. D. Froggatt, I. G. Knowles and R. G. Moorhouse, Phys. Lett. B 249 (1990) 273.

[59] C. D. Froggatt, I. G. Knowles and R. G. Moorhouse, Nucl. Phys. B 386 (1992) 63.

[60] C. D. Froggatt, R. Nevzorov, H. B. Nielsen and D. Thompson, Phys. Lett. B 657 (2007) 95 [arXiv:0708.2903 [hep-ph]].

[61] C. D. Froggatt, R. Nevzorov and H. B. Nielsen, J. Phys. Conf. Ser. 110 (2008) 062010 arXiv:0708.2905 [hep-ph]].

[62] C. D. Froggatt, R. Nevzorov and H. B. Nielsen, in Proceedings of the 15th International Conference on Supersymmetry and the Unification of Fundamental Interactions, Karlsruhe, Germany, 2007, p.710 [arXiv:0710.2457 [hep-ph]].

[63] C. T. Hill, Phys. Rev. D 24 (1981) 691.

[64] R. Tarrach, Nucl. Phys. B 183 (1981) 384.

[65] N. Gray, D. J. Broadhurst, W. Grafe and K. Schilcher, Z. Phys. C 48 (1990) 673. 
[66] D. J. Broadhurst, N. Gray and K. Schilcher, Z. Phys. C 52 (1991) 111.

[67] K. G. Chetyrkin and M. Steinhauser, Phys. Rev. Lett. 83 (1999) 4001.

[68] K. G. Chetyrkin and M. Steinhauser, Nucl. Phys. B 573 (2000) 617.

[69] B. Schrempp and F. Schrempp, Phys. Lett. B 299 (1993) 321.

[70] B. Schrempp, Phys. Lett. B 344 (1995) 193.

[71] B. Schrempp and M. Wimmer, Prog. Part. Nucl. Phys. 37 (1996) 1.

[72] R. B. Nevzorov and M. A. Trusov, Phys. Atom. Nucl. 64 (2001) 1299 [Yad. Fiz. 64 (2001) 1375].

[73] R. B. Nevzorov and M. A. Trusov, Phys. Atom. Nucl. 65 (2002) 335 [Yad. Fiz. 65 (2002) 359].

[74] R. B. Nevzorov, K. A. Ter-Martirosyan and M. A. Trusov, arXiv:hep-ph/0301068.

[75] Z. Z. Xing, H. Zhang and S. Zhou, Phys. Rev. D 77 (2008) 113016 arXiv:0712.1419 [hep-ph]].

[76] M. Ciuchini, G. Degrassi, P. Gambino, G. F. Giudice, Nucl. Phys. B 527 (1998) 21.

[77] P. Gambino, M. Misiak, Nucl. Phys. B 611 (2001) 338.

[78] P. A. Kovalenko, R. B. Nevzorov and K. A. Ter-Martirosian, Phys. Atom. Nucl. 61 (1998) 812 [Yad. Fiz. 61 (1998) 898].

[79] R. B. Nevzorov and M. A. Trusov, J. Exp. Theor. Phys. 91 (2000) 1079 [Zh. Eksp. Teor. Fiz. 91 (2000) 1251].

[80] R. B. Nevzorov, K. A. Ter-Martirosyan and M. A. Trusov, Phys. Atom. Nucl. 65 (2002) 285 [Yad. Fiz. 65 (2002) 311].

[81] D. J. Miller, R. Nevzorov and P. M. Zerwas, Nucl. Phys. B 681 (2004) 3.

[82] R. Nevzorov and D. J. Miller, arXiv:hep-ph/0411275.

[83] S. F. King, S. Moretti and R. Nevzorov, Phys. Rev. D 73 (2006) 035009.

[84] For a recent review, see: M. Carena and H. E. Haber, Prog. Part. Nucl. Phys. 50 (2003) 63. 


\section{Figure captions}

Fig.1. (a) The top quark Yukawa coupling at $\mu=M_{t}$ versus the MPP scale $\Lambda$. (b) The dependence of $\tan \beta$ on the scale $\Lambda$. The solid and dashed curves correspond to $h_{t}^{2}(\Lambda)=10$ and $h_{t}^{2}(\Lambda)=2.25$. The dash-dotted lines represent the quasi-fixed point solution (31). Here we set $m_{t}\left(M_{t}\right)=161.6 \mathrm{GeV}$ and $\alpha_{3}\left(M_{Z}\right)=0.117$. The MPP scale $\Lambda$ is given in $\mathrm{GeV}$.

Fig.2. The allowed range of $R_{1}(\Lambda)$ and $R_{2}(\Lambda)$ for (a) $\Lambda=M_{P l}$ and (b) $\Lambda=10 \mathrm{TeV}$. The solid line corresponds to the upper bound on $R_{2}(\Lambda)$, which comes from the requirement that $\lambda_{4}^{2}(\Lambda) \geq 0$. The dotted curve represents the lower bound on $R_{2}(\Lambda)$ caused by the vacuum stability condition. The open circle indicates the position of the fixed point in the gaugeless limit. Other parameters are fixed as follows: $h_{t}(\Lambda)=3, m_{t}\left(M_{t}\right)=161.6 \mathrm{GeV}$ and $\alpha_{3}\left(M_{Z}\right)=0.117$.

Fig.3. The renormalisation group flow of $R_{i}(\mu)$ from the Planck scale to the electroweak scale in (a) the $\left(R_{1}, R_{2}\right)$ plane, (b) the $\left(R_{1}, R_{3}\right)$ plane and (c) the $\left(R_{1}, R_{4}\right)$ plane, for different initial values of $R_{1}(\Lambda)$ and $R_{2}(\Lambda)$ from the allowed part of parameter space (see Fig.2). The open circle indicates the position of the quasi-fixed point (47). Here we set $h_{t}(\Lambda)=3$. The initial values of $\lambda_{3}(\Lambda)$ and $\lambda_{4}(\Lambda)$ satisfy the MPP conditions (48) and (49).

Fig.4. The dependence of (a) $R_{1}\left(M_{t}\right)$, (b) $R_{2}\left(M_{t}\right)$, (c) $R_{3}\left(M_{t}\right)$ and (d) $R_{4}\left(M_{t}\right)$ on the MPP scale near the quasi-fixed point. Solid and dashed lines correspond to $h_{t}^{2}(\Lambda)=10$ and $h_{t}^{2}(\Lambda)=2.25$ respectively. The Higgs self-couplings $\lambda_{1}(\Lambda)$ and $\lambda_{2}(\Lambda)$ are fixed so that $R_{1}(\Lambda)=0.75$ and $R_{2}(\Lambda)=0.883$, whereas $\lambda_{3}(\Lambda)$ and $\lambda_{4}(\Lambda)$ obey the MPP conditions (48) and (49).

Fig.5. Higgs masses and couplings for $\Lambda=M_{P l}, h_{t}^{2}\left(M_{P l}\right)=10, R_{1}\left(M_{P l}\right)=0.75$ and $R_{2}\left(M_{P l}\right)=0.883$. (a) The dependence of the spectrum of Higgs bosons on the pseudoscalar Higgs mass $m_{A}$. The dash-dotted and dashed lines correspond to the $\mathrm{CP}$-even Higgs boson masses, while the solid line represents the mass of the charged Higgs states. All masses are given in GeV. (b) Absolute values of the relative couplings $R_{Z Z h_{i}}$ of the Higgs scalars to $Z$ pairs. The solid and dashed-dotted curves represent the dependence of the couplings of the lightest and heaviest $\mathrm{CP}$-even Higgs states to $\mathrm{Z}$ pairs on $m_{A}$. (c) Absolute values of the relative couplings $R_{t \bar{t} h_{i}}$ of the lightest (solid curve) and heaviest (dashed-dotted curve) $\mathrm{CP}$-even Higgs bosons to the top quark as a function of $m_{A}$. Here the Higgs self-couplings $\lambda_{3}(\Lambda)$ and $\lambda_{4}(\Lambda)$ satisfy the MPP conditions (48) and (49). 
Fig.6. Upper bound on the mass of the SM-like Higgs boson versus the MPP scale $\Lambda$ in the quasi-fixed point scenario. The solid and dashed curves correspond to $h_{t}^{2}(\Lambda)=10$ and $h_{t}^{2}(\Lambda)=2.25$. The Higgs self-couplings $\lambda_{1}(\Lambda)$ and $\lambda_{2}(\Lambda)$ are fixed so that $R_{1}(\Lambda)=0.75$ and $R_{2}(\Lambda)=0.883$, whereas $\lambda_{3}(\Lambda)$ and $\lambda_{4}(\Lambda)$ obey the MPP conditions (48) and (49). The value of $\tan \beta$ is chosen so that $m_{t}\left(M_{t}\right)=161.6 \mathrm{GeV}$. The MPP scale $\Lambda$ is given in $\mathrm{GeV}$.

Fig.7. Higgs masses and couplings for $\Lambda=100 \mathrm{TeV}, h_{t}^{2}(\Lambda)=10, R_{1}(\Lambda)=0.75$ and $R_{2}(\Lambda)=0.883$. (a) Spectrum of Higgs bosons versus $m_{A}$. (b) Absolute values of the relative couplings $R_{Z Z h_{i}}$ of the Higgs scalars to $Z$ pairs. (c) Absolute values of the relative couplings $R_{t \bar{t} h_{i}}$ of the $\mathrm{CP}-$ even Higgs bosons to the top quark as a function of $m_{A}$. Here $\lambda_{3}(\Lambda)$ and $\lambda_{4}(\Lambda)$ obey MPP conditions. The notations are the same as in Fig. 5.

Fig.8. The running of $\lambda_{1}(\mu), \lambda_{2}(\mu)$ and $\widehat{\lambda}(\mu)$ in the MPP scenario which implies the existence of a set of vacua degenerate with respect to $\tan \gamma$ at the scale $\Lambda$. (a) The renormalisation group flow of these Higgs self-couplings below $\Lambda=M_{P l}$. (b) The evolution of $\lambda_{1}(\mu), \lambda_{2}(\mu)$ and $\widehat{\lambda}(\mu)$ below $\Lambda=10 \mathrm{TeV}$. The solid, dashed and dash-dotted lines correspond to $\lambda_{1}(\mu), \lambda_{2}(\mu)$ and $\widehat{\lambda}(\mu)$ respectively. Here we fix $g_{b}(\Lambda)=g_{\tau}(\Lambda)=\lambda_{5}(\Lambda)=0$, $m_{t}\left(M_{t}\right)=165 \mathrm{GeV}$ and $\alpha_{3}\left(M_{Z}\right)=0.117$. Other parameters are specified in Table 3 . 


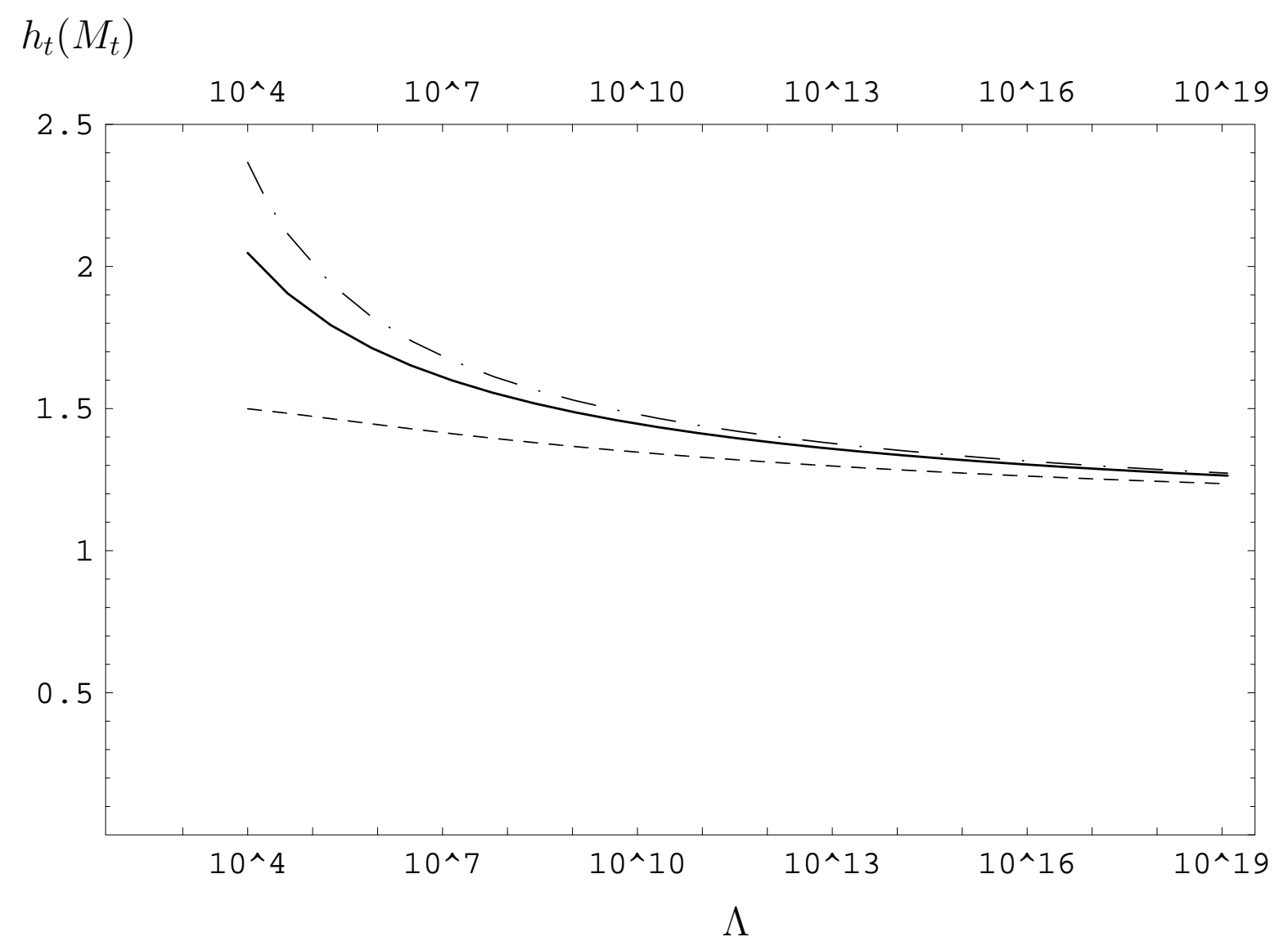

Fig.1a

$\tan \beta$

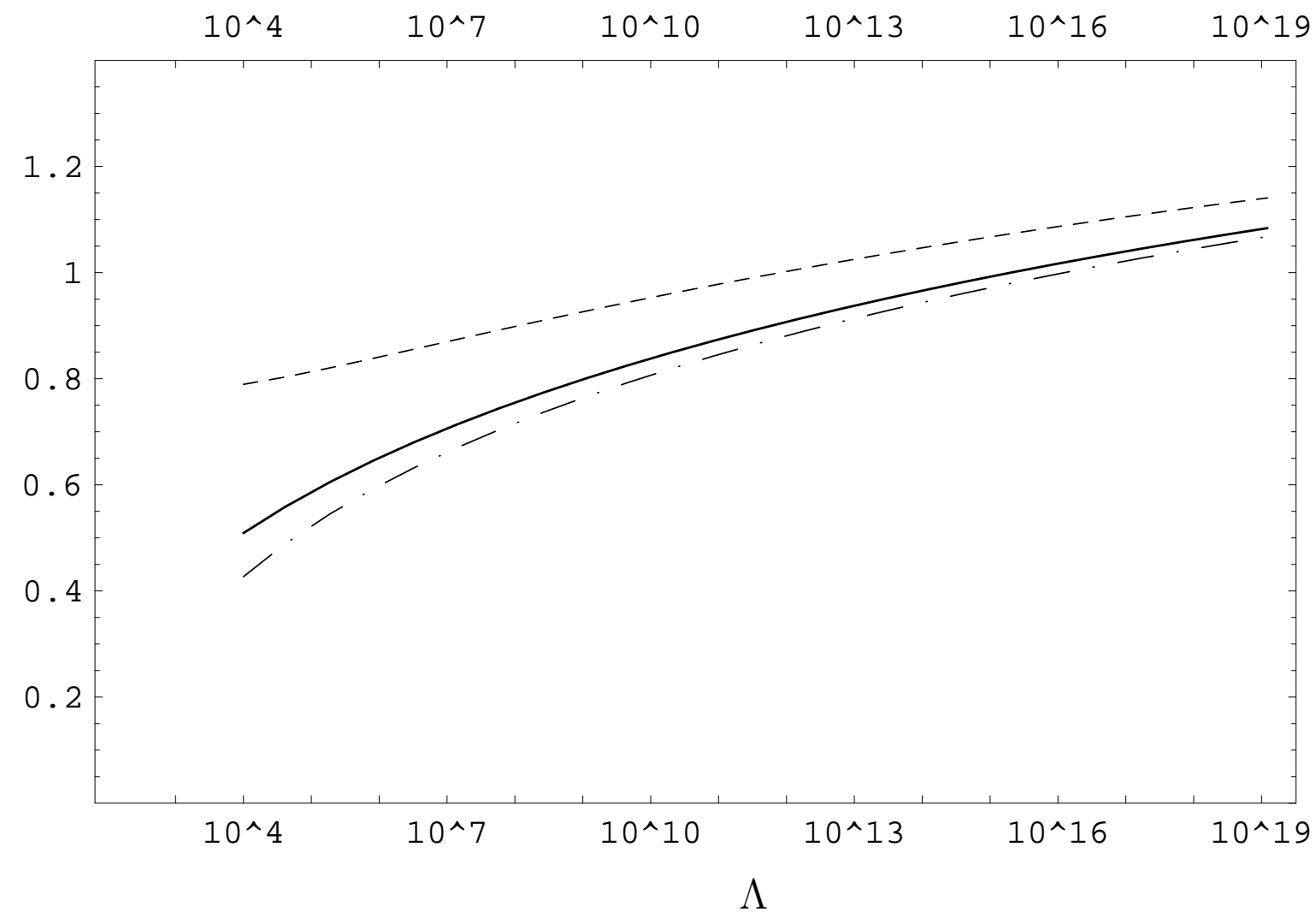

Fig.1b 


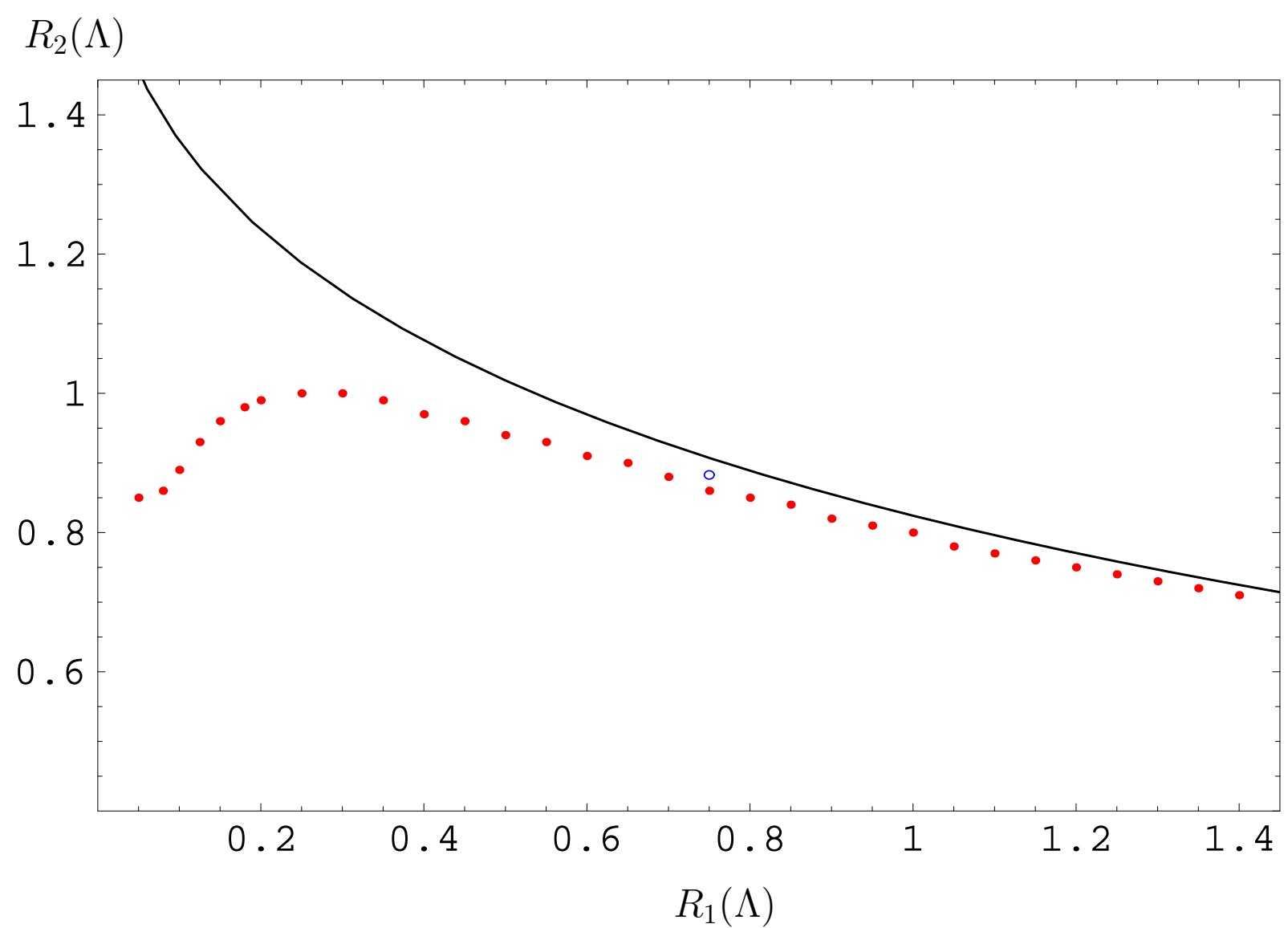

Fig.2a

$R_{2}(\Lambda)$

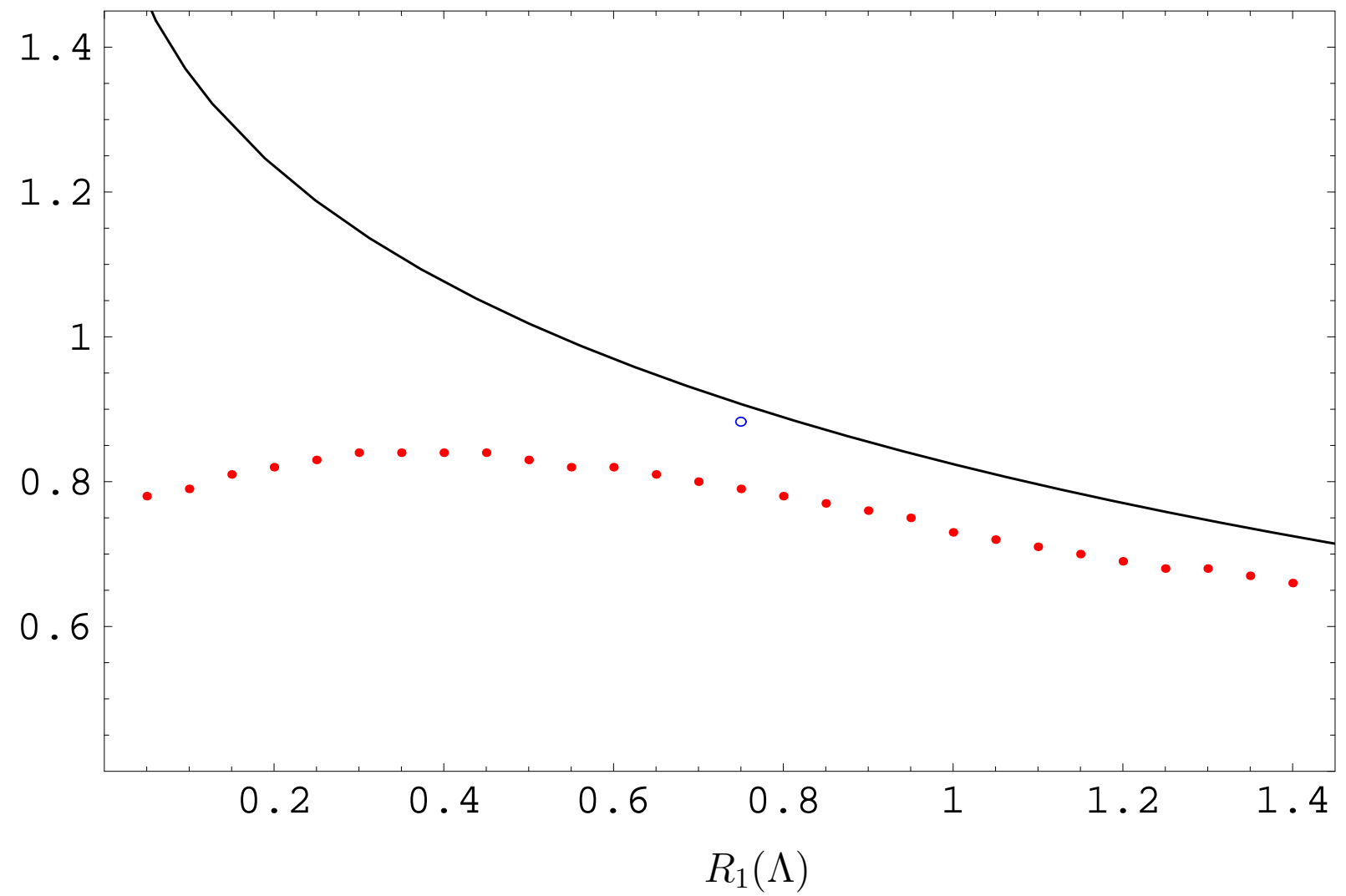

Fig. 2b 


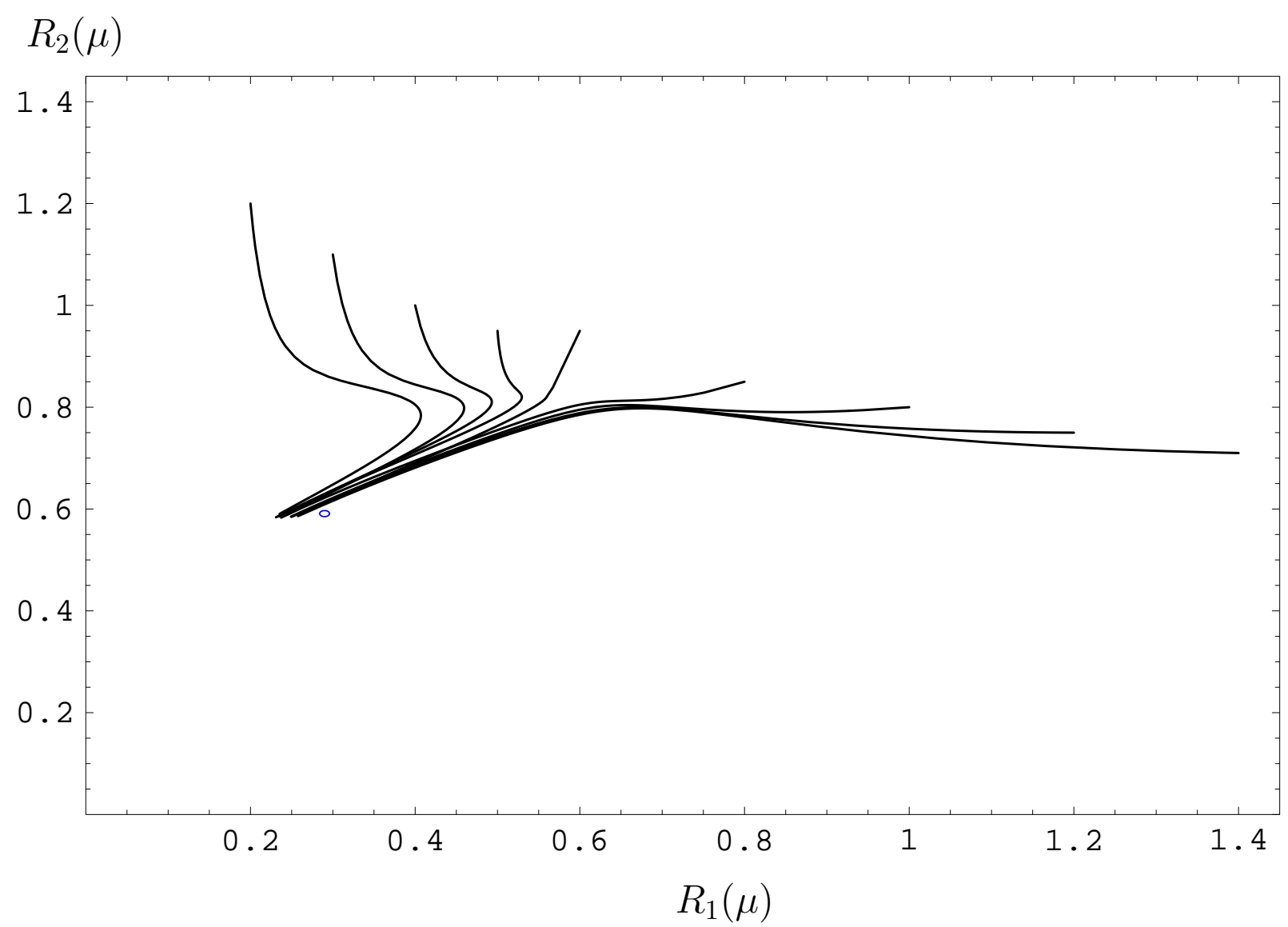

Fig.3a

$R_{3}(\mu)$

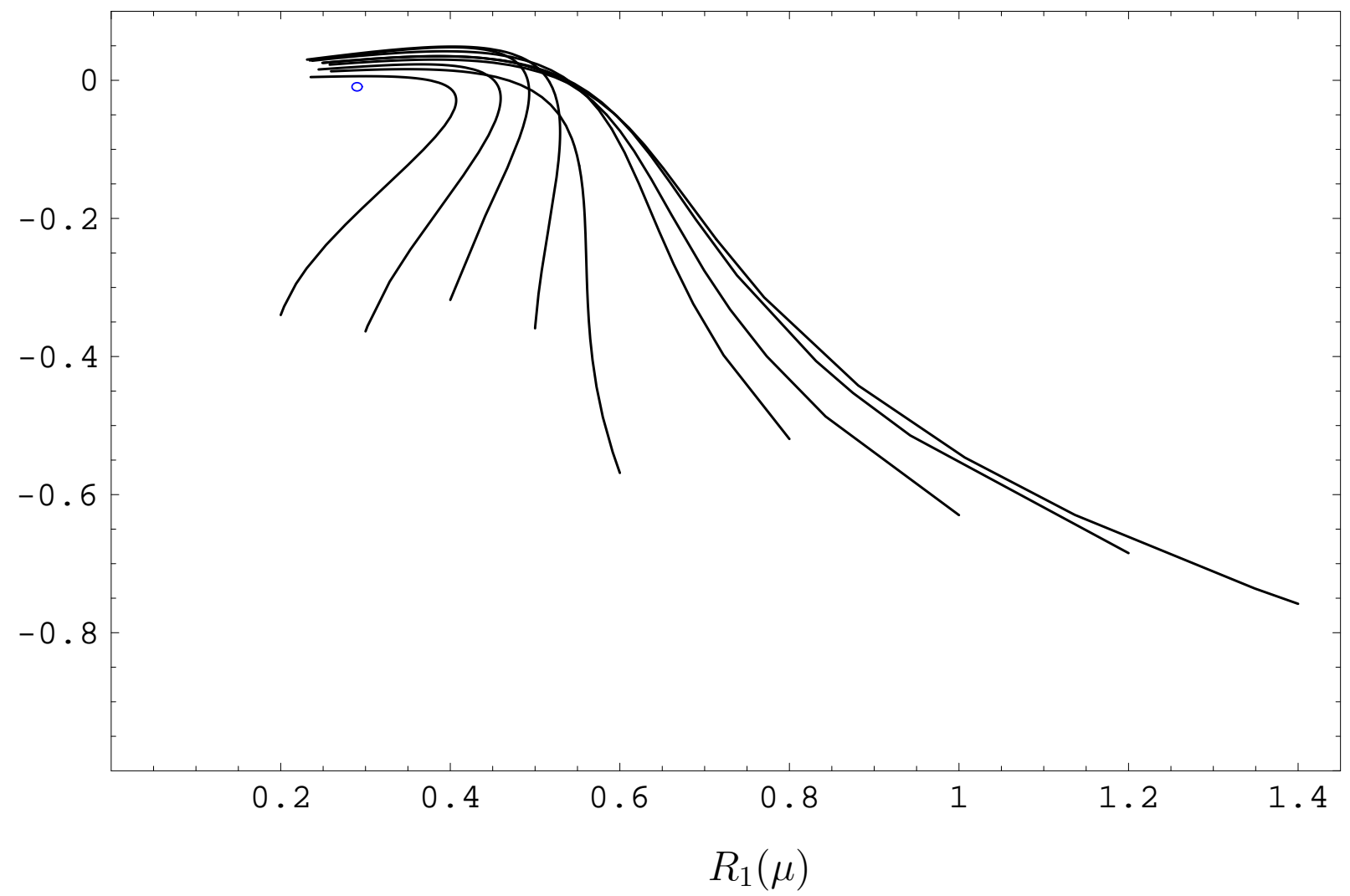

Fig.3b 


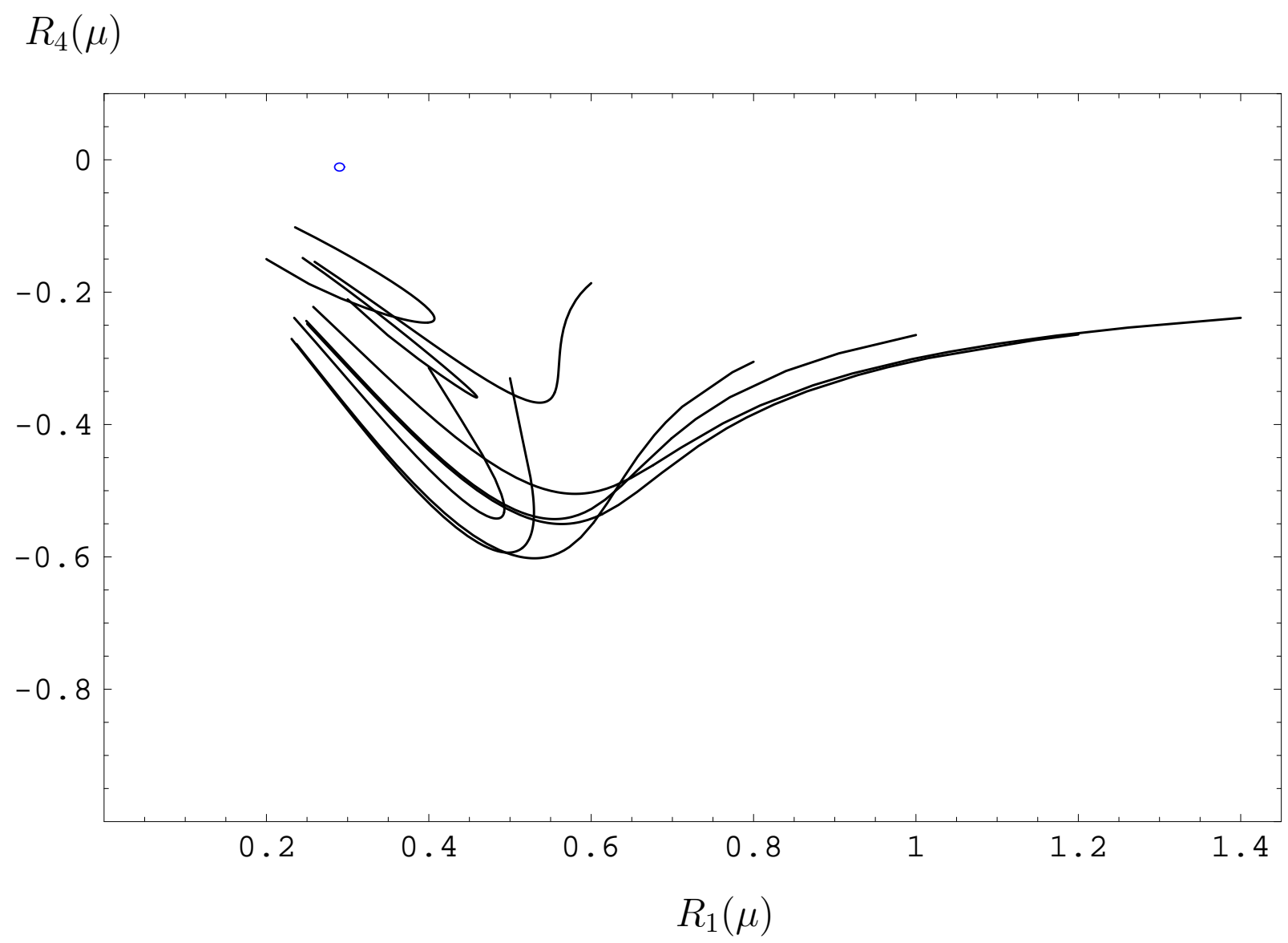

Fig.3c 


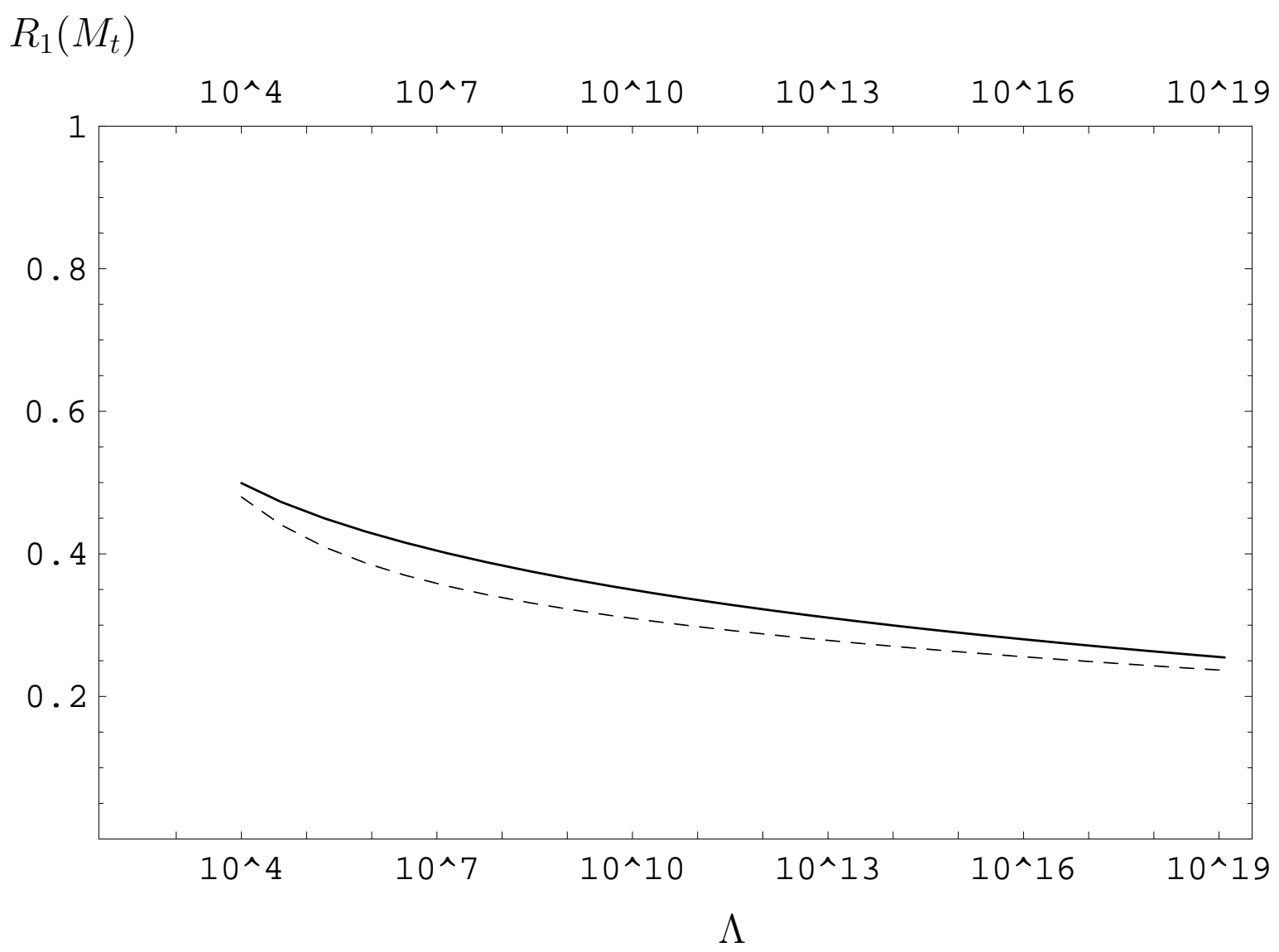

Fig.4a

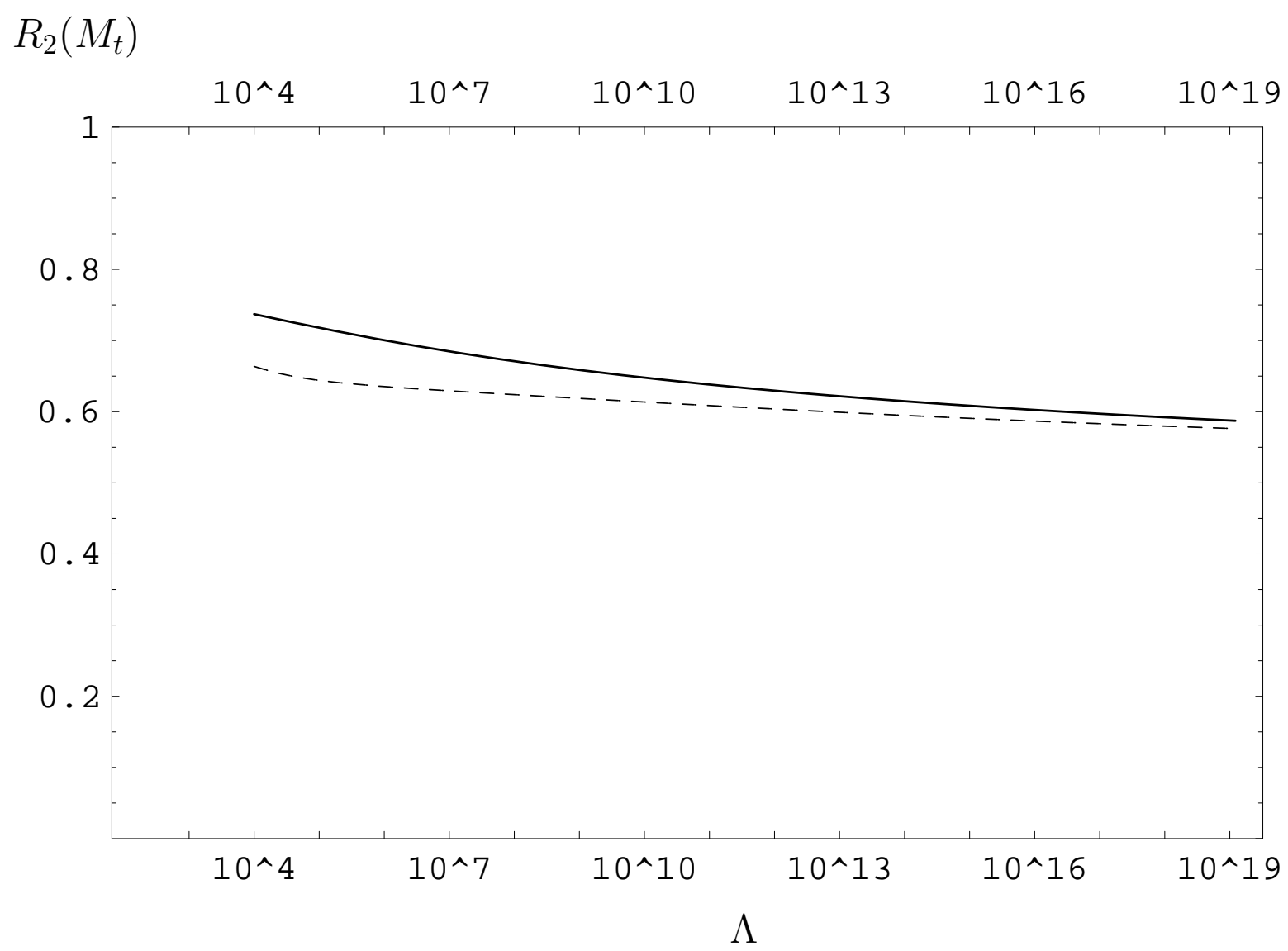

Fig.4b 


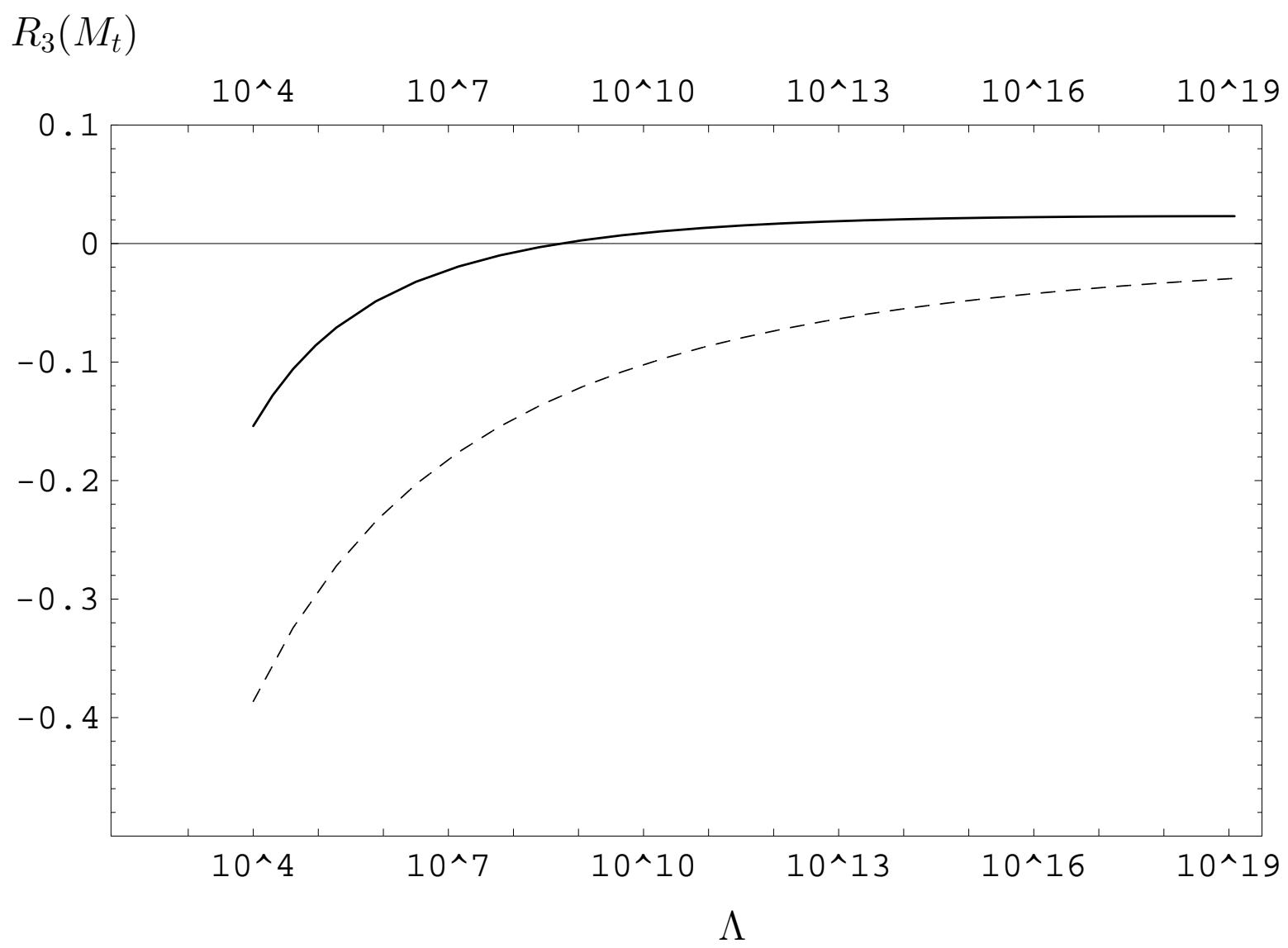

Fig.4c

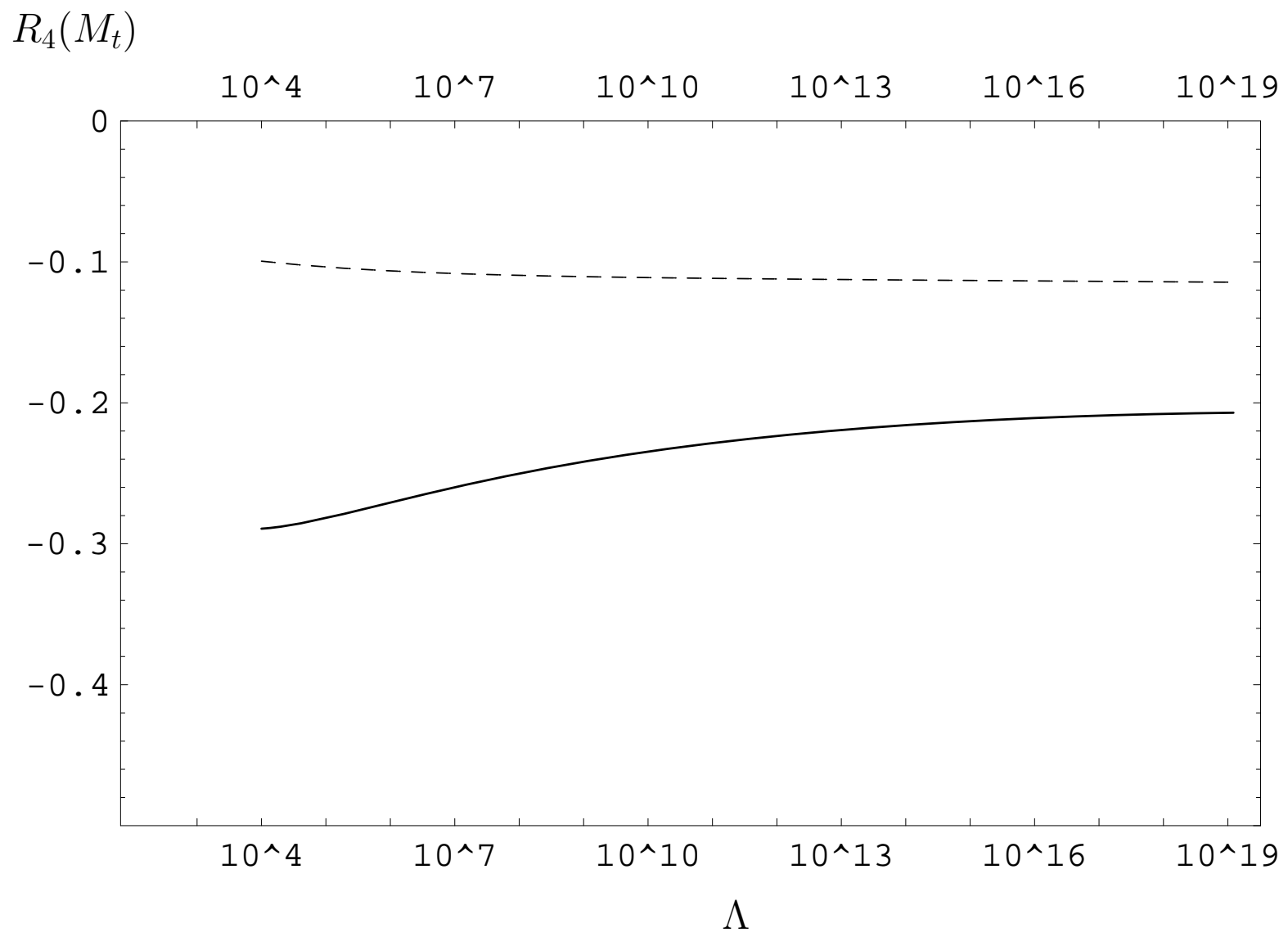

Fig.4d 


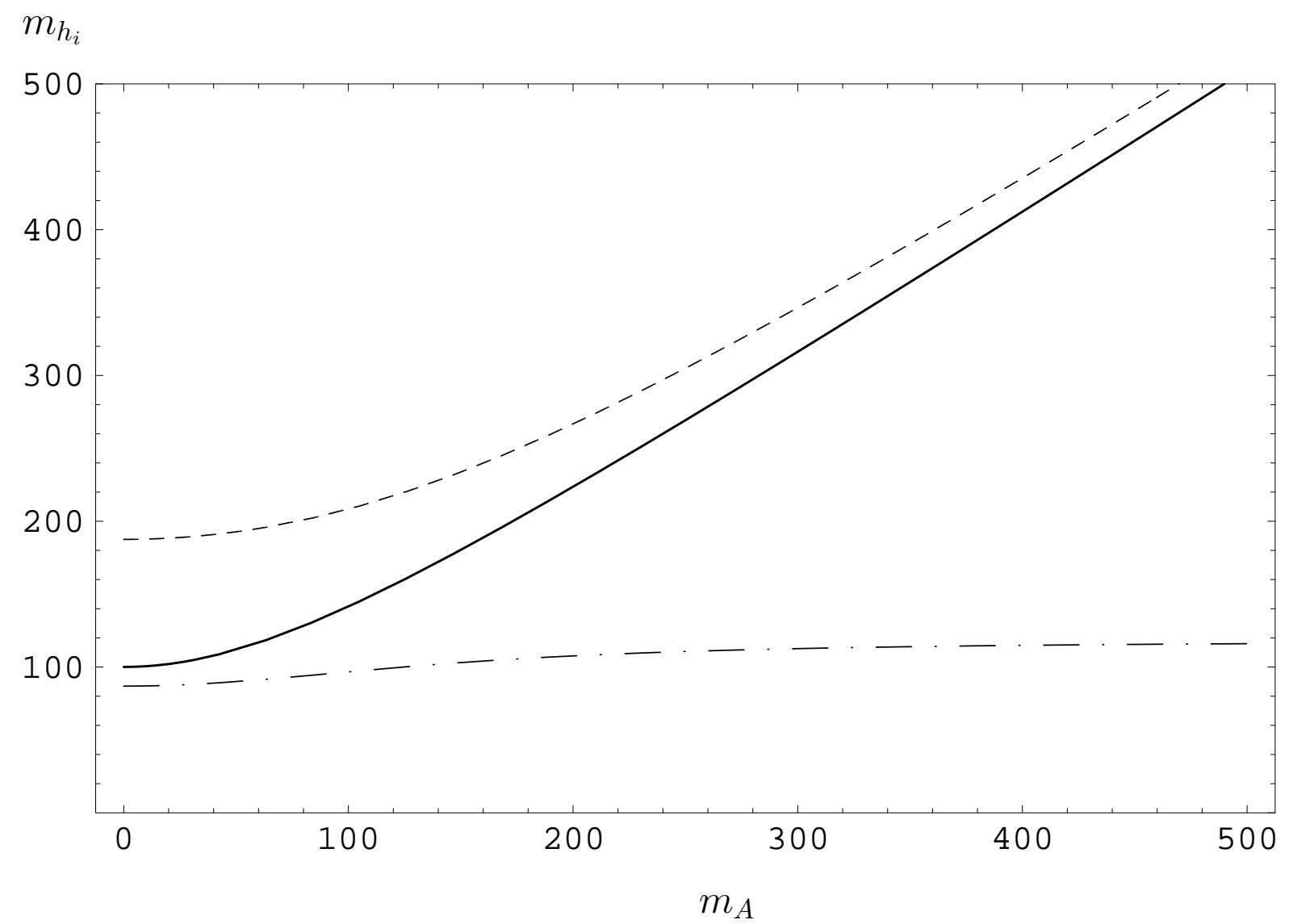

$\left|R_{Z Z h_{i}}\right| \quad$ Fig.5a

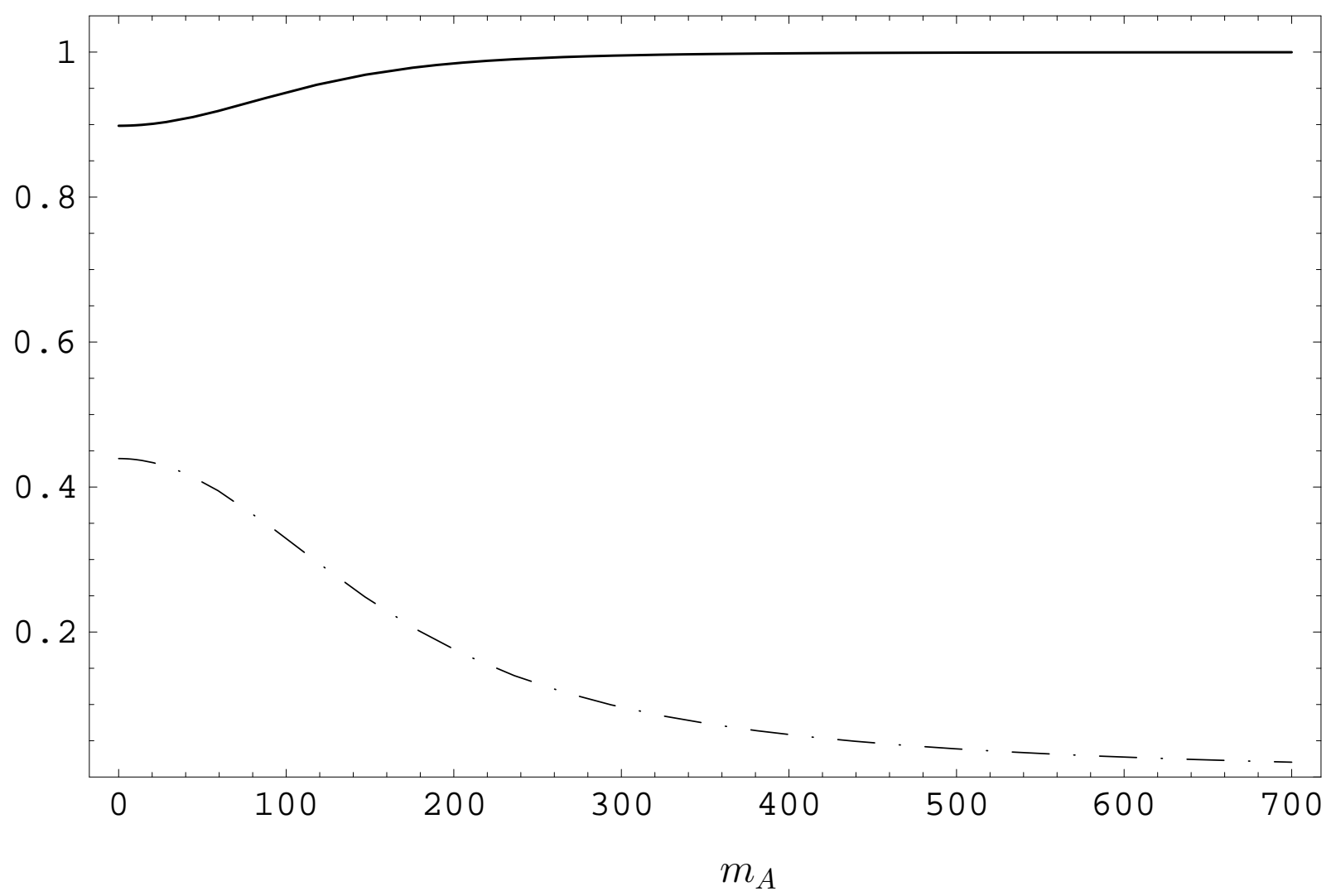

Fig.5b 


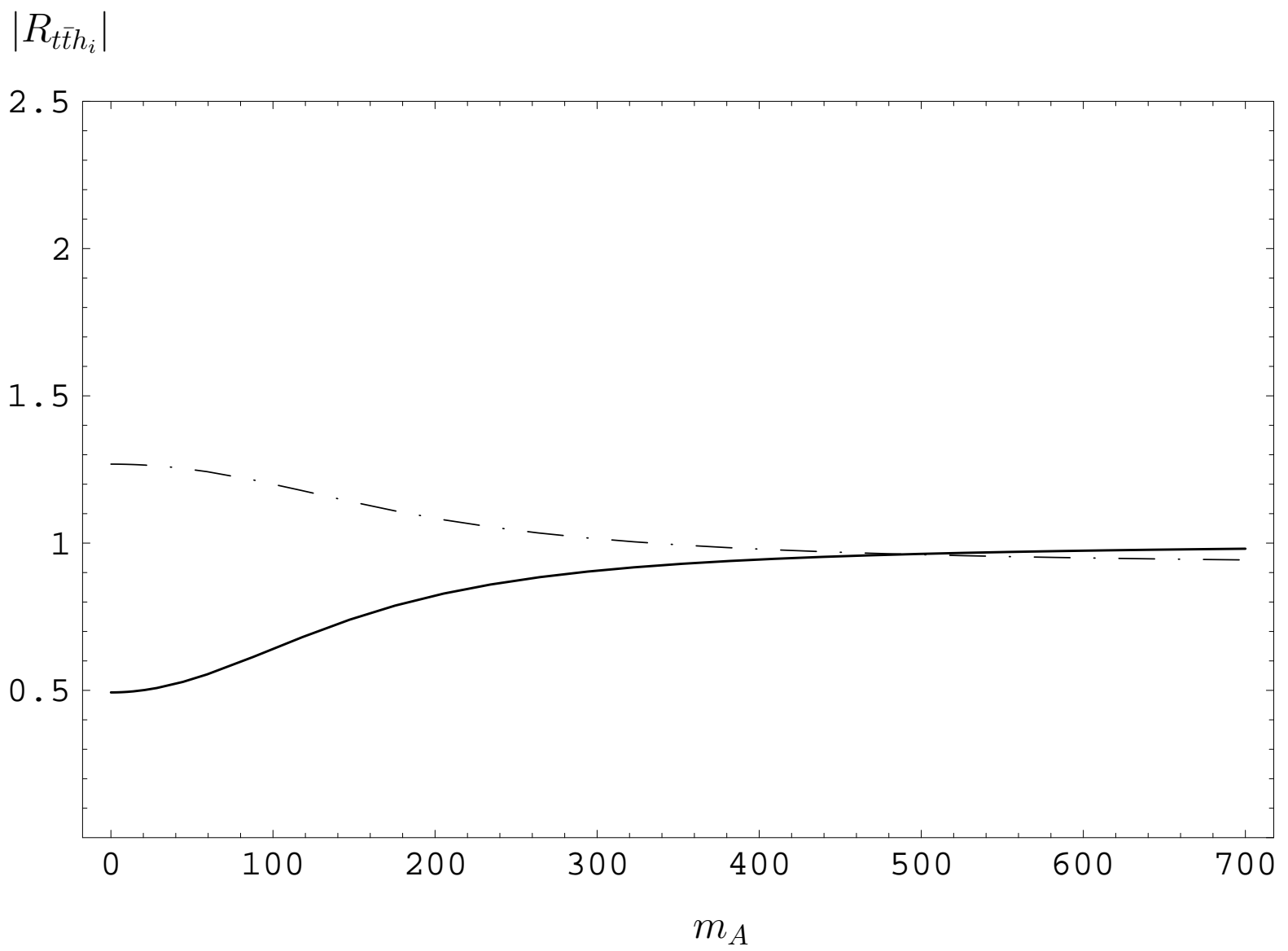

Fig.5c 


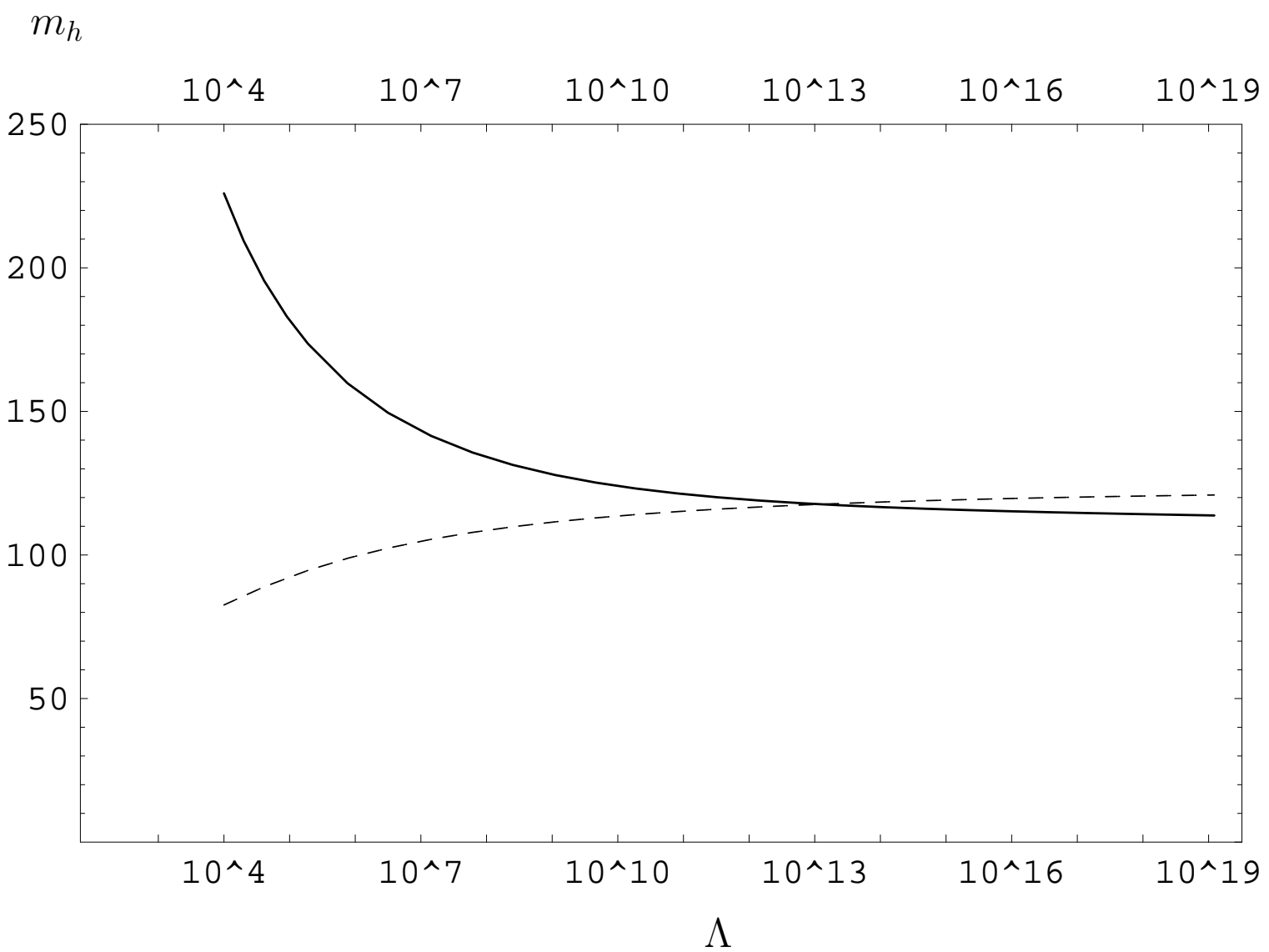

Fig.6 


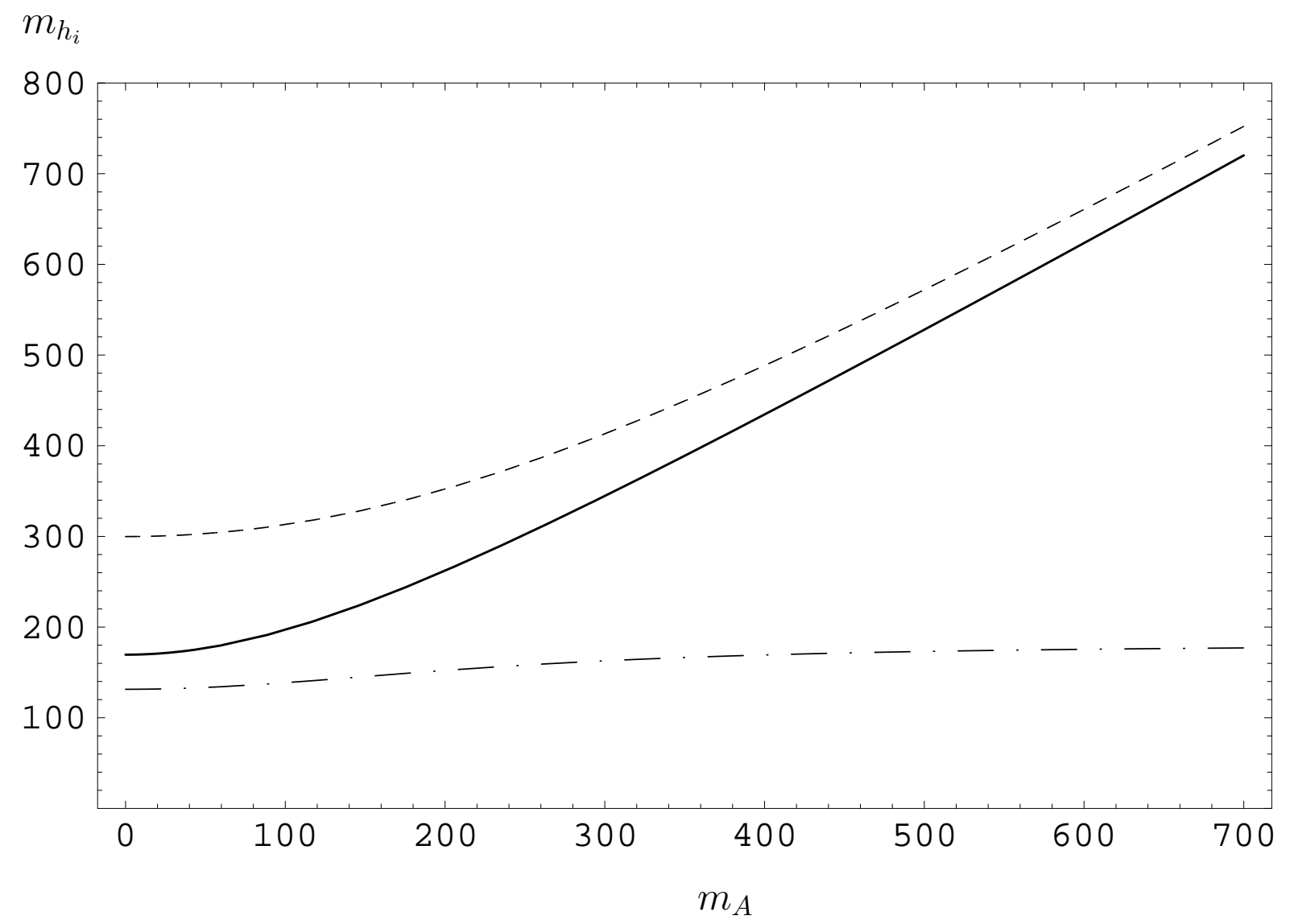

$\left|R_{Z Z h_{i}}\right|$

Fig.7a

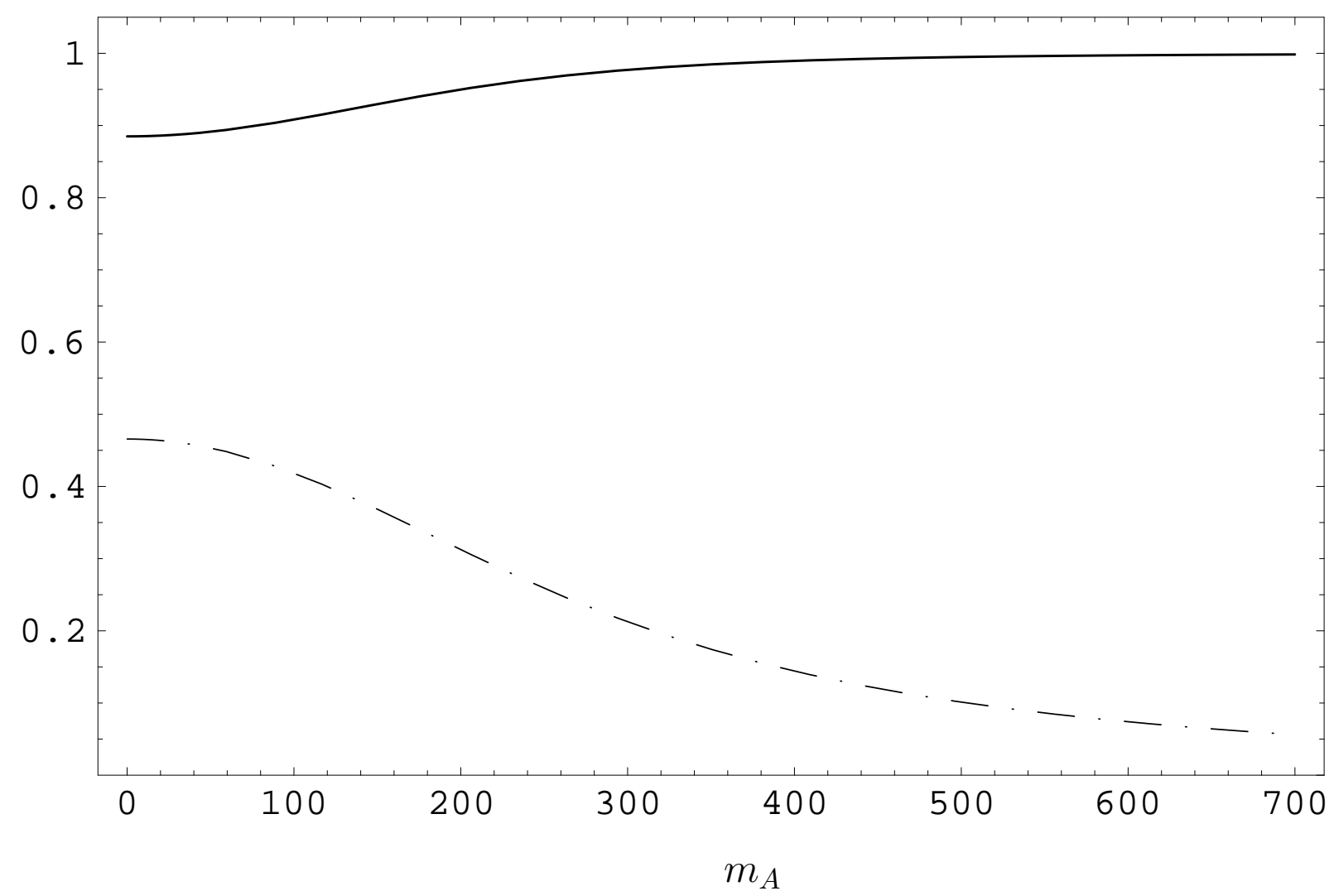

Fig. 7b 


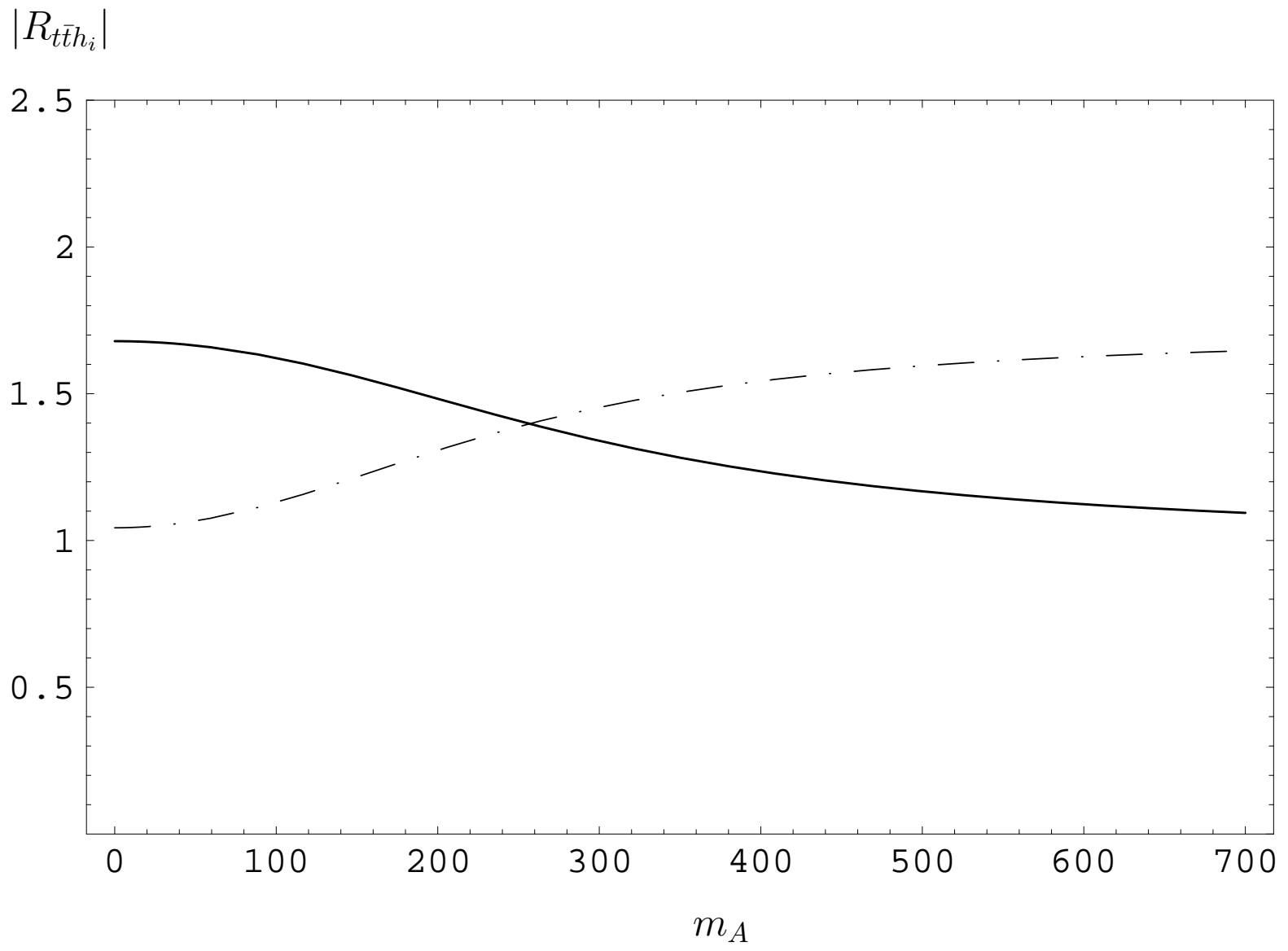

Fig.7c 


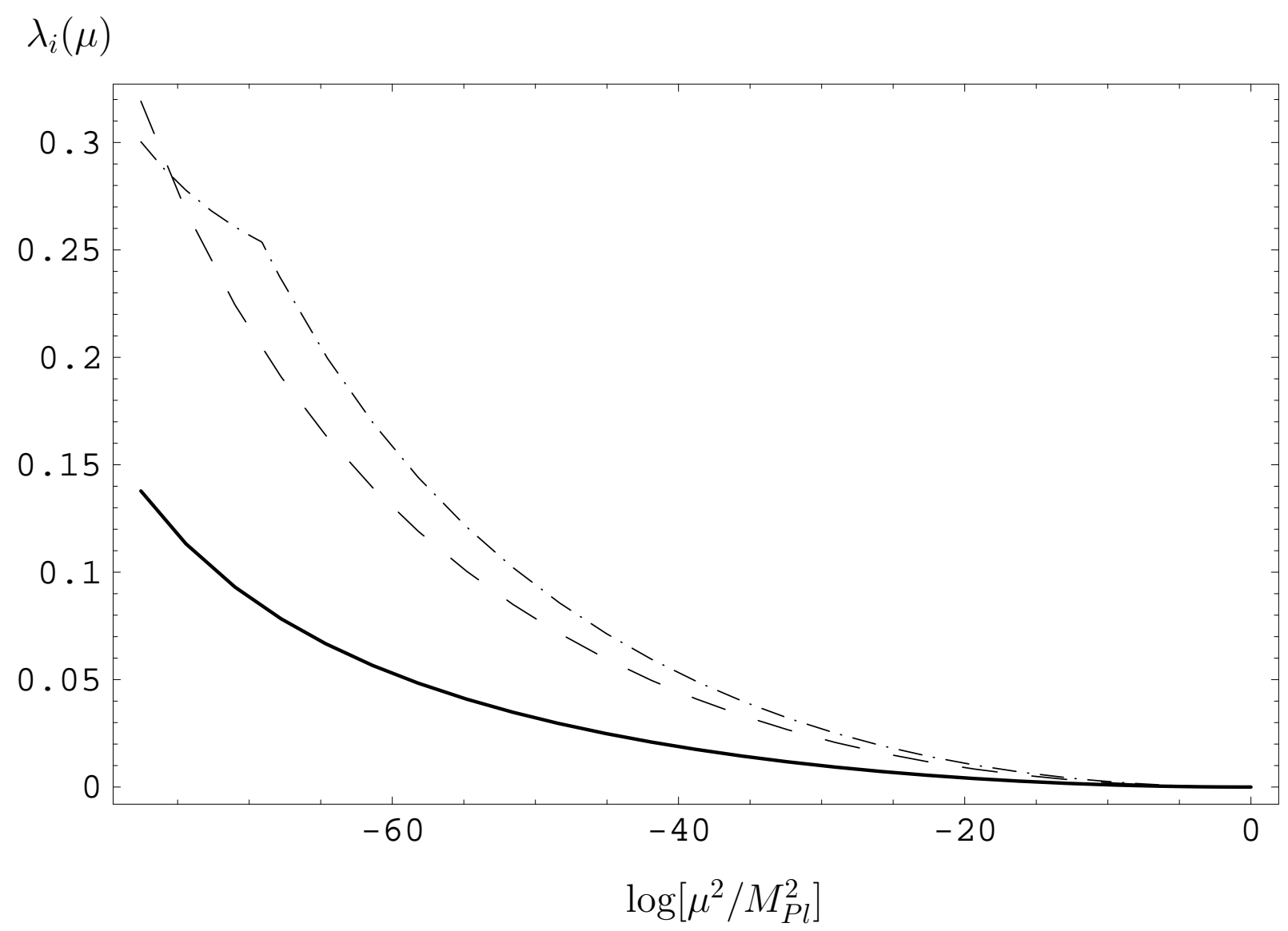

Fig. 8a

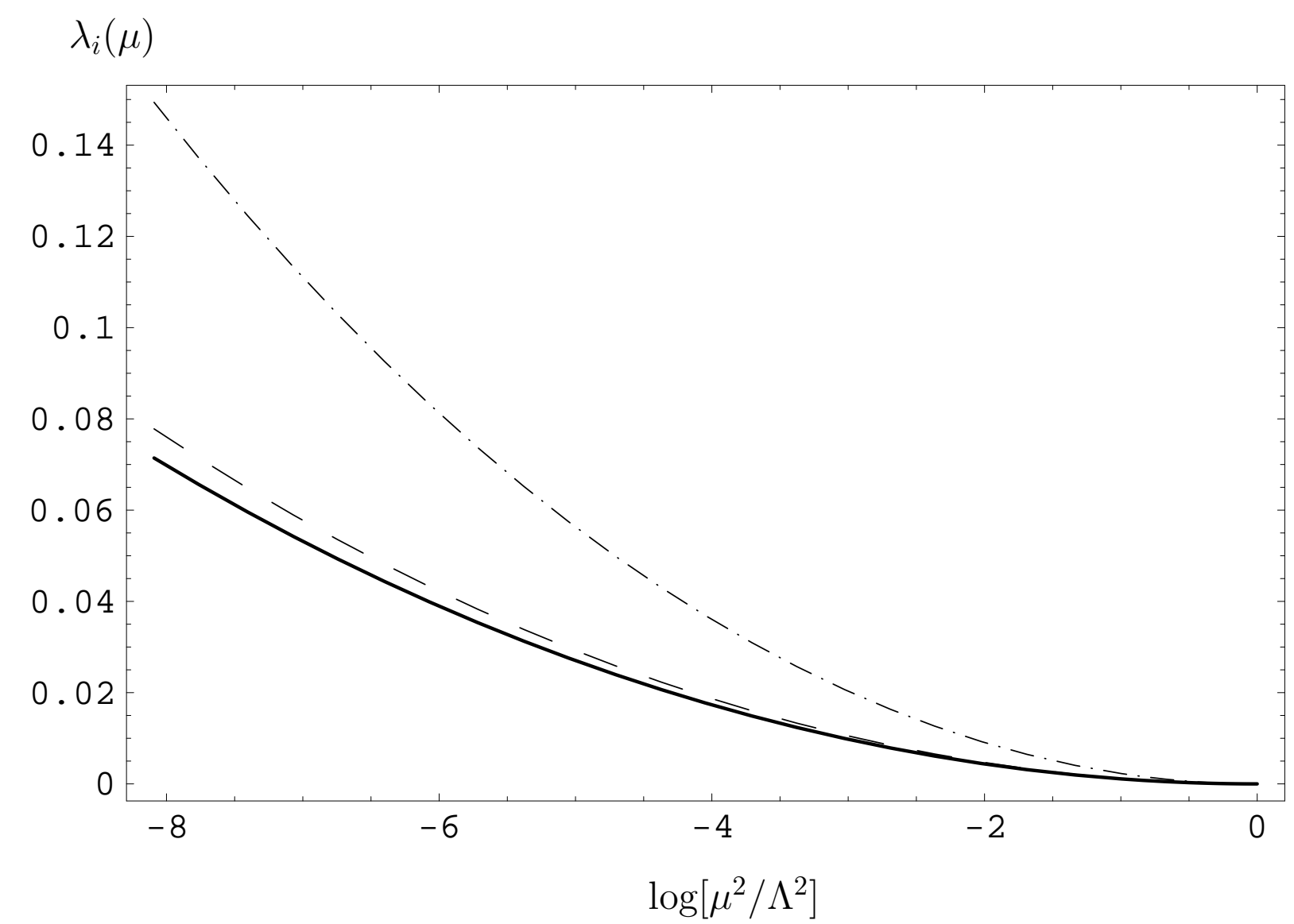

Fig.8b 


\begin{tabular}{|c|c|c|c|c|c|c|}
\hline & $\Lambda$ & $\rho_{t}\left(M_{t}\right)$ & $\rho_{b}\left(M_{t}\right)$ & $\rho_{\tau}\left(M_{t}\right)$ & $m_{t}\left(M_{t}\right)$ & $m_{b}\left(M_{t}\right)$ \\
\hline \multirow{4}{*}{ model $I I$} & $M_{P l}$ & 1.012 & 0.707 & 0.391 & $213-203$ & $2.39-2.09$ \\
\cline { 2 - 7 } & $10^{13} \mathrm{GeV}$ & 1.173 & 0.782 & 0.586 & $229-207$ & $2.05-1.79$ \\
\cline { 2 - 7 } & $10^{7} \mathrm{GeV}$ & 1.664 & 0.986 & 1.287 & $273-208$ & $1.56-1.34$ \\
\hline \multirow{4}{*}{ model $I I I$} & $M_{P l}$ & 1.081 & - & 0.732 & $220-208$ & - \\
\cline { 2 - 7 } & $10^{13} \mathrm{GeV}$ & 1.248 & - & 1.002 & $237-212$ & - \\
\cline { 2 - 7 } & $10^{7} \mathrm{GeV}$ & 1.754 & - & 1.868 & $281-211$ & - \\
\hline \multirow{3}{*}{ model $I V$} & $M_{P l}$ & 0.976 & 0.949 & - & $209-199$ & - \\
\cline { 2 - 7 } & $10^{13} \mathrm{GeV}$ & 1.128 & 1.107 & - & $225-203$ & - \\
\cline { 2 - 7 } & $10^{7} \mathrm{GeV}$ & 1.593 & 1.578 & - & $267-205$ & - \\
\hline
\end{tabular}

Table 1: Predictions for $\rho_{t}\left(M_{t}\right)=\left(h_{t}\left(M_{t}\right) / g_{3}\left(M_{t}\right)\right)^{2}, \rho_{b}\left(M_{t}\right)=\left(h_{b}\left(M_{t}\right) / g_{3}\left(M_{t}\right)\right)^{2}$, $\rho_{\tau}\left(M_{t}\right)=\left(h_{\tau}\left(M_{t}\right) / g_{3}\left(M_{t}\right)\right)^{2}$ and running quark masses obtained near the quasi-fixed points of the RG equations at large values of $\tan \beta$, in different MPP scenarios with approximate Peccei-Quinn and $Z_{2}$-symmetries. All masses are given in $\mathrm{GeV}$. The specified ranges of quark masses correspond to the variation of $h_{t}^{2}(\Lambda)$ from 10 to 1.

\begin{tabular}{|c|c|c|c|}
\hline$R_{1}^{0}$ & $R_{2}^{0}$ & $R_{3}^{0}$ & $R_{4}^{0}$ \\
\hline 0 & -1.133 & 0 & 0 \\
\hline 0 & 0.883 & 0 & 0 \\
\hline 0.750 & -1.133 & 0 & 0 \\
\hline 0.750 & 0.883 & 0 & 0 \\
\hline 0.742 & 0.880 & -0.160 & 0.259 \\
\hline 0.742 & 0.880 & 0.099 & -0.259 \\
\hline
\end{tabular}

Table 2: The six fixed points of the RG equations for $g_{1}(\mu)=g_{2}(\mu)=g_{3}(\mu)=0$ and $h_{b}(\mu)=h_{\tau}(\mu)=g_{b}(\mu)=g_{\tau}(\mu)=g_{t}(\mu)=0$.

\begin{tabular}{|c|c|c|c|c|}
\hline$\Lambda$ & $h_{t}(\Lambda)$ & $\tan \beta$ & $m_{b}\left(M_{t}\right)$ & $m_{h}$ \\
\hline $10^{4} \mathrm{GeV}$ & 0.811 & 49.84 & 3.24 & 69.0 \\
\hline $10^{8} \mathrm{GeV}$ & 0.645 & 47.64 & 3.28 & 115.7 \\
\hline $10^{12} \mathrm{GeV}$ & 0.549 & 47.41 & 3.18 & 130.1 \\
\hline $10^{16} \mathrm{GeV}$ & 0.480 & 48.53 & 2.94 & 136.3 \\
\hline$M_{P l}$ & 0.435 & 50.43 & 2.61 & 138.9 \\
\hline
\end{tabular}

Table 3: The dependence of the upper bound $m_{h}$ for the lightest Higgs mass, $\tan \beta$ and $m_{b}\left(M_{t}\right)$ on the MPP scale, in the MPP scenario that implies the existence of a set of vacua degenerate with respect to $\tan \gamma$ at the scale $\Lambda$. The Peccei-Quinn symmetry violating Yukawa couplings $g_{b}(\Lambda)$ and $g_{\tau}(\Lambda)$, as well $\lambda_{5}(\Lambda)$, are set to zero. All masses are given in $\mathrm{GeV}$. 


\begin{tabular}{|c|c|c|c|}
\hline$\Lambda$ & $h_{t}(\Lambda)$ & $\frac{\lambda_{5}(\Lambda)}{\lambda_{4}(\Lambda)}$ & $m_{h}$ \\
\hline $10^{4} \mathrm{GeV}$ & 0.811 & 0 & 69.0 \\
\hline $10^{4} \mathrm{GeV}$ & 0.811 & 0.5 & 72.4 \\
\hline $10^{4} \mathrm{GeV}$ & 0.811 & 0.8 & 74.3 \\
\hline $10^{4} \mathrm{GeV}$ & 0.811 & -0.5 & 72.1 \\
\hline $10^{4} \mathrm{GeV}$ & 0.811 & -0.8 & 73.9 \\
\hline $10^{8} \mathrm{GeV}$ & 0.645 & 0 & 115.7 \\
\hline $10^{8} \mathrm{GeV}$ & 0.645 & 0.5 & 117.9 \\
\hline $10^{8} \mathrm{GeV}$ & 0.645 & 0.8 & 119.1 \\
\hline $10^{8} \mathrm{GeV}$ & 0.645 & -0.5 & 117.9 \\
\hline $10^{8} \mathrm{GeV}$ & 0.645 & -0.8 & 119.0 \\
\hline$M_{P l}$ & 0.435 & 0 & 138.9 \\
\hline$M_{P l}$ & 0.435 & 0.5 & 139.2 \\
\hline$M_{P l}$ & 0.435 & 0.8 & 139.2 \\
\hline$M_{P l}$ & 0.435 & -0.5 & 139.2 \\
\hline$M_{P l}$ & 0.435 & -0.8 & 139.2 \\
\hline & \multicolumn{2}{|l}{} \\
\hline
\end{tabular}

Table 4: The dependence of the upper bound $m_{h}$ for the lightest Higgs mass on the Higgs self-couplings $\lambda_{5}(\Lambda)$ and $\lambda_{4}(\Lambda)$ for different values of the scale $\Lambda$, in the MPP scenario that implies the existence of a set of vacua degenerate with respect to $\tan \gamma$ at the MPP scale. The Peccei-Quinn symmetry violating Yukawa couplings $g_{b}(\Lambda)$ and $g_{\tau}(\Lambda)$ are set to zero. The theoretical restriction on the SM-like Higgs mass is given in GeV. 